\title{
Proton Abstraction Mechanism in the Palladium-Catalyzed Intramolecular Arylation: Substituent Effects
}

Domingo García-Cuadrado, Paula de Mendoza, Ataualpa A. C. Braga, Feliu Maseras* and Antonio M. Echavarren*

Institute of Chemical Research of Catalonia (ICIQ), Av. Països Catalans 16, 43007 Tarragona, Catalonia, Spain

fmaseras@iciq.es; aechavarren@iciq.es

\section{Contents}

1 Computational Details

2 Justification of the choice of methods and models

3 References

4 Cartesian Coordinates (in $\AA$ ) and Absolute Energies (in a.u.).

\section{Computational Details}

All calculations were performed with Gaussian03. ${ }^{1}$ The methods were B3LYP ${ }^{2}$ and ONIOM(B3LYP:UFF). ${ }^{3,4}$ Two basis sets were used for the QM calculations. In basis set I, the inner electrons of $\mathrm{Pd}$ and $\mathrm{Br}$ were described by an effective core potential (LANL2DZ), and the associated double- $\zeta$ basis set was used for the outer electrons. ${ }^{5} \mathrm{~A} d$ polarization shell was added in the case of Br. ${ }^{6}$ The $6-31 \mathrm{G}(\mathrm{d})$ basis set was used for $\mathrm{H}, \mathrm{C}, \mathrm{O}, \mathrm{Si}, \mathrm{P}^{7}$ In basis set II, diffuse functions were added to most of the atoms. The $6-31+\mathrm{G}(\mathrm{d})$ set was used for $\mathrm{H}, \mathrm{C}, \mathrm{O}$, $\mathrm{Si}, \mathrm{P}^{8}$ and the basis set for Br was also expanded. ${ }^{9}$

The initial calculations on systems t1, with $\mathrm{PH}_{3}$ phosphines, were carried out at the B3LYP level with basis set I. For the calculations on systems t2, with the real phosphine ligands, the ONIOM(B3LYP:UFF) approach was used, with the phosphine substituents in the MM region. The starting phosphine conformation was taken from reported X-ray structures of similar systems. ${ }^{10}$ The geometry optimization for the $\mathbf{t} \mathbf{2}$ systems was carried out with basis set I, and the reported energetics were obtained through single point calculations with basis set II. Solvation effects in DMA ( $=37.8$, radius $=2.642 \mathrm{~A})$ were evaluated through singlepoint $\mathrm{PCM}$ calculations ${ }^{11}$ on the system defined by the QM region of the total system. The energetics presented for systems $\mathbf{t} \mathbf{2}$ are obtained thus as $\mathrm{E}_{\mathrm{ONIOM}}(\mathrm{TOTAL})+\left(\mathrm{E}_{\text {solvated }}\right.$ (model) - E(model)). 


\section{Justification of the choice of methods and models}

Basis set I, essentially double- $\zeta$ with polarization, was used in the preliminary calculations on the model system because it was the same we have used in the initial communication on this same topic. We decided to check basis set II, including diffuse functionts, to account for the presence of anionic ligands in the system. Table S1 collects the computed potential energy barriers obtained with different basis sets for the two assisted mechanisms on the $\mathbf{t} 1 \mathbf{a}$ and $\mathbf{t} \mathbf{1} \mathbf{b}$ systems. Values obtained with basis set II on the structures optimized with basis set I are also included. The trends are in all cases conserved, and the precise values are quite close, with spans of $1.5,0.8 \mathrm{kcal} / \mathrm{mol}$ for the intramolecular mechanism; and spans of 2.4, $3.6 \mathrm{kcal} / \mathrm{mol}$ for the intermolecular mechanism. We consider the values with the two basis sets sufficiently similar to keep the validity of the qualitative analysis with basis set I on the model system. On the other hand, we find the values sufficiently different to warrant the use of the larger basis set for the analysis of the substituent effects. We did not replace basis set I in the presentation of the first set of calculations in order to conserve the same set of values of the initial communication.

Table S1: Values computed with different basis sets for the potential energy barriers $(\mathrm{kcal} / \mathrm{mol})$ involved in the assisted intramolecular and sssisted intermolecular mechanisms on t1a and $\mathbf{t} \mathbf{t} \mathbf{b}$ model systems.

\begin{tabular}{lccccccc}
\hline & \multicolumn{3}{c}{ Assisted intramolecular } & & \multicolumn{3}{c}{ Assisted intermolecular } \\
\cline { 2 - 4 } \cline { 6 - 8 } system & Basis I & Basis II & Basis II//Basis I & & Basis I & Basis II & Basis II//Basis I \\
\hline $\mathbf{t 1 a}$ & 23.5 & 25.0 & 24.8 & & 17.4 & 19.8 & 19.0 \\
$\mathbf{t 1 b}$ & 13.2 & 14.0 & 13.8 & & 14.4 & 18.0 & 16.6 \\
\hline$\Delta(\mathbf{t} 1 \mathbf{b}-\mathbf{t 1 b})$ & 10.3 & 11.0 & 11.0 & & 3.0 & 1.8 & 2.4 \\
\hline \hline
\end{tabular}

We used hydrogencarbonate as a base because it is surely present in solution, and because the use of other alternative bases on model systems $\mathbf{t} \mathbf{1} \mathbf{a}, \mathbf{t} \mathbf{1} \mathbf{b}$ produced insatisfactory results. For the intramolecular mechanism, carbonate coordinates in a $\kappa^{2}$ mode in the intermediate, and produces very little discrimination between $\mathbf{t} \mathbf{1} \mathbf{a}, \mathbf{t} \mathbf{1} \mathbf{b}$ in terms of energy barrier (difference lower than $1 \mathrm{kcal} / \mathrm{mol}$ ). We attribute this result to the exaggerated basicity of the carbonate anion holding two full charges. We tried to overcome this problem by adding a potassium cation, but this increased conformational complexity because the electrostatic interaction of potassium is usually not limited to a single anionic center. For the intermolecular mechanism, we found that carbonate spontaneously displaces the phosphine ligand, leading to a different type of mechanism with a phosphine-less palladium complex that seems unrealistic. We do not claim that these results prove that hydrogencarbonate is the active form of the base in this system. We think instead that the faulty results are at least in part associated to limitations in our computational approach. In particular, they could be probably solved (or at least atenuated) with the use of geometry optimizations in solution, the introduction of explicit solvent molecules and the introduction of additional molecules of base or counteranions. However, some of these methodological features are not available in the program we used, and others would require a substantial increase in computer time. We have thus settled on hydrogencarbonate constitutes a reasonable representative of the behavior of the base that is not plagued by the computational 
problems associated to carbonate or potassium hydrogencarbonate. 


\section{References}

(1) Frisch, M. J.; Trucks, G. W.; Schlegel, H. B.; Scuseria, G. E.; Robb, M. A.; Cheeseman, J. R.; Montgomery, Jr., J. A.; Vreven, T.; Kudin, K. N.; Burant, J. C.; Millam, J. M.; Iyengar, S. S.; Tomasi, J.; Barone, V.; Mennucci, B.; Cossi, M.; Scalmani, G.; Rega, N.; Petersson, G. A.; Nakatsuji, H.; Hada, M.; Ehara, M.; Toyota, K.; Fukuda, R.; Hasegawa, J.; Ishida, M.; Nakajima, T.; Honda, Y.; Kitao, O.; Nakai, H.; Klene, M.; Li, X.; Knox, J. E.; Hratchian, H. P.; Cross, J. B.; Bakken, V.; Adamo, C.; Jaramillo, J.; Gomperts, R.; Stratmann, R. E.; Yazyev, O.; Austin, A. J.; Cammi, R.; Pomelli, C.; Ochterski, J. W.; Ayala, P. Y.; Morokuma, K.; Voth, G. A.; Salvador, P.; Dannenberg, J. J.; Zakrzewski, V. G.; Dapprich, S.; Daniels, A. D.; Strain, M. C.; Farkas, O.; Malick, D. K.; Rabuck, A. D.; Raghavachari, K.; Foresman, J. B.; Ortiz, J. V.; Cui, Q.; Baboul, A. G.; Clifford, S.; Cioslowski, J.; Stefanov, B. B.; Liu, G.; Liashenko, A.; Piskorz, P.; Komaromi, I.; Martin, R. L.; Fox, D. J.; Keith, T.; Al-Laham, M. A.; Peng, C. Y.; Nanayakkara, A.; Challacombe, M.; Gill, P. M. W.; Johnson, B.; Chen, W.; Wong, M. W.; Gonzalez, C.; and Pople, J. A.; "Gaussian 03, Revision C.02", Gaussian, Inc., Wallingford, CT, 2004.

(2) (a) Lee, C.; Parr, R. G.; Yang, W. Phys. Rev. 1988, 37, 785-789. (b) Becke, A. D. J. Phys. Chem. 1993, 98, 5648-5652. (c) Stephens, P. J.; Devlin, F. J.; Chabalowski, C. F.; Frisch, M. J. J. Phys. Chem. 1994, 98, 11623-11627.

(3) (a) Maseras, F.; Morokuma, K. J. Comput. Chem. 1995, 16, 1170-1179. (b) Dapprich, S.; Komaromi, I.; Byun, K. S.; Morokuma, K.; Frisch, M. J. J. Mol. Struct.-Theochem 1999, 461, 1-21.

(4) Rappe, A. K.; Casewit, C. J.; Colwell, K. S.; Goddard, W. A.; Skiff, W. M. J. Am. Chem. Soc. 1992, 114, 10024-10035.

(5) (a) Hay, P. J.; Wadt, W. R. J. Chem. Phys. 1985, 82, 299-310. (b) Wadt, W. R.; Hay, P. J. J. Chem. Phys. 1985, 82, 284-298.

(6) Höllwarth, A.; Böhme, M.; Dapprich, S.; Ehlers, A. W.; Gobbi, A.; Jonas, V.; Köhler, K. F.; Stegmann, R.; Veldkamp, A.; Frenking, G. Chem. Phys. Lett. 1993, 208, 237-240.

(7) (a) Francl, M. M.; Pietro, W. J.; Hehre, W. J.; Binkley, J. S.; Gordon, M. S.; Defrees, D. J.; Pople, J. A. J. Chem. Phys. 1982, 77, 3654-3665.

(8) Clark, T.; Chandrasekhar, J.; Spitznagel, G. W.; Schleyer, P. V. J. Comput. Chem. 1983, 4, 294-301.

(9) Check, C. E.; Faust, T. O.; Bailey, J. M.; Wright, B. J.; Gilbert, T. M.; Sunderlin, L. S. J. Phys. Chem. A 2001, 105, 8111-8116.

(10) Dotta, P.; Kumar, P. G. A.; Pregosin, P. S.; Albinati, A.; Rizzato, S. Organometallics 2004, 23, 2995-2304.

(11) Miertus, S.; Scrocco, E.; Tomasi, J. J. Chem. Phys. 1981, 55, 117-129. 


\section{Cartesian Coordinates (in $\AA$ ) and Absolute Energies (in a.u.).}

Table S2: Cartesian coordinates $(\AA)$ of $\mathbf{t 1 a R}$ for the unassisted mechanism $(\mathrm{E}=-1024.43109688$ a.u.).

\begin{tabular}{lrrr}
\hline \hline $\mathrm{C}$ & 3.140061 & -1.821249 & -1.509948 \\
$\mathrm{C}$ & 1.798794 & -1.436148 & -1.402931 \\
$\mathrm{C}$ & 1.299002 & -0.907136 & -0.207271 \\
$\mathrm{C}$ & 2.150879 & -0.769641 & 0.905738 \\
$\mathrm{C}$ & 3.489623 & -1.173311 & 0.785735 \\
$\mathrm{C}$ & 3.985995 & -1.698458 & -0.408054 \\
$\mathrm{Pd}$ & -0.656444 & -0.329977 & -0.075528 \\
$\mathrm{C}$ & 1.676334 & -0.093673 & 2.175458 \\
$\mathrm{C}$ & 1.807716 & 1.460218 & 2.095197 \\
$\mathrm{C}$ & 1.266268 & 2.008998 & 0.797899 \\
$\mathrm{C}$ & -0.125211 & 1.993241 & 0.534716 \\
$\mathrm{C}$ & -0.619159 & 2.389714 & -0.725133 \\
$\mathrm{C}$ & 0.265531 & 2.809099 & -1.725379 \\
$\mathrm{C}$ & 1.631507 & 2.851614 & -1.456282 \\
$\mathrm{C}$ & 2.125223 & 2.447910 & -0.210191 \\
$\mathrm{P}$ & -1.301496 & -2.495948 & -0.170434 \\
$\mathrm{H}$ & -1.956267 & -2.990097 & 0.972610 \\
$\mathrm{H}$ & 1.146996 & -1.546854 & -2.266893 \\
$\mathrm{H}$ & -0.355012 & -3.524035 & -0.378150 \\
$\mathrm{H}$ & -2.245378 & -2.824195 & -1.160955 \\
$\mathrm{H}$ & -1.691307 & 2.394729 & -0.890106 \\
$\mathrm{H}$ & -0.116028 & 3.117004 & -2.694432 \\
$\mathrm{H}$ & 2.325493 & 3.188546 & -2.222050 \\
$\mathrm{H}$ & 3.196681 & 2.458278 & -0.028623 \\
$\mathrm{H}$ & 4.150875 & -1.069221 & 1.644709 \\
$\mathrm{H}$ & 5.024907 & -2.010690 & -0.474959 \\
$\mathrm{H}$ & 3.514056 & -2.223443 & -2.448542 \\
$\mathrm{H}$ & -0.830157 & 1.809742 & 1.344437 \\
$\mathrm{H}$ & 2.251685 & -0.445356 & 3.040572 \\
$\mathrm{H}$ & 0.625546 & -0.346023 & 2.362607 \\
$\mathrm{H}$ & 2.865350 & 1.732002 & 2.188437 \\
$\mathrm{H}$ & 1.282460 & 1.899703 & 2.953155 \\
$\mathrm{Br}$ & -3.223613 & 0.132314 & 0.176904 \\
\hline \hline & & & \\
\hline
\end{tabular}


Table S3: Cartesian coordinates $(\AA)$ of t1aTS for the unassisted mechanism $(\mathrm{E}=$ 1024.36219852 a.u.).

\begin{tabular}{lrrr}
\hline \hline $\mathrm{C}$ & 3.848475 & -0.696833 & -1.295567 \\
$\mathrm{C}$ & 2.456088 & -0.843661 & -1.351029 \\
$\mathrm{C}$ & 1.675578 & -0.530001 & -0.235802 \\
$\mathrm{C}$ & 2.271055 & -0.039968 & 0.937803 \\
$\mathrm{C}$ & 3.665096 & 0.097272 & 0.976206 \\
$\mathrm{C}$ & 4.453555 & -0.229697 & -0.129674 \\
$\mathrm{Pd}$ & -0.327835 & -0.810866 & -0.199523 \\
$\mathrm{C}$ & 1.405599 & 0.365612 & 2.111861 \\
$\mathrm{C}$ & 0.792442 & 1.777849 & 1.973128 \\
$\mathrm{C}$ & 0.181805 & 2.179405 & 0.634824 \\
$\mathrm{C}$ & -0.400663 & 1.281732 & -0.291378 \\
$\mathrm{C}$ & -0.924217 & 1.802010 & -1.495575 \\
$\mathrm{C}$ & -0.902923 & 3.166048 & -1.783872 \\
$\mathrm{C}$ & -0.339209 & 4.045880 & -0.859883 \\
$\mathrm{C}$ & 0.194889 & 3.549767 & 0.328045 \\
$\mathrm{P}$ & -0.074927 & -3.189747 & -0.227326 \\
$\mathrm{H}$ & 0.700700 & -3.784272 & 0.793469 \\
$\mathrm{H}$ & 1.992643 & -1.202216 & -2.266471 \\
$\mathrm{H}$ & 0.594392 & -3.774984 & -1.326523 \\
$\mathrm{H}$ & -1.171790 & -4.083762 & -0.175756 \\
$\mathrm{H}$ & 0.644486 & 4.242520 & 1.037407 \\
$\mathrm{H}$ & 4.136442 & 0.468061 & 1.884952 \\
$\mathrm{H}$ & 5.533453 & -0.117126 & -0.079531 \\
$\mathrm{H}$ & 4.451847 & -0.948872 & -2.164503 \\
$\mathrm{H}$ & 1.992080 & 0.347508 & 3.039174 \\
$\mathrm{H}$ & 0.601448 & -0.369722 & 2.234240 \\
$\mathrm{H}$ & 1.571435 & 2.513749 & 2.208621 \\
$\mathrm{H}$ & 0.031185 & 1.892132 & 2.760083 \\
$\mathrm{Br}$ & -3.147313 & -0.454739 & 0.379565 \\
$\mathrm{H}$ & -1.980226 & 0.554665 & 0.241351 \\
$\mathrm{H}$ & -1.375229 & 1.123370 & -2.216497 \\
$\mathrm{H}$ & -1.319022 & 3.535030 & -2.717938 \\
$\mathrm{H}$ & -0.309012 & 5.113367 & -1.063436 \\
\hline \hline & & &
\end{tabular}


Table S4: Cartesian coordinates $(\AA)$ of $\mathbf{t 1 a R}$ for the intramolecular assisted mechanism $(\mathrm{E}=$ -1275.62814306 a.u.).

\begin{tabular}{lrrr}
\hline \hline $\mathrm{C}$ & 3.022209 & -2.038702 & -1.416218 \\
$\mathrm{C}$ & 1.716742 & -1.537409 & -1.378708 \\
$\mathrm{C}$ & 1.192677 & -0.983022 & -0.204469 \\
$\mathrm{C}$ & 1.988066 & -0.939151 & 0.958252 \\
$\mathrm{C}$ & 3.289624 & -1.461848 & 0.908672 \\
$\mathrm{C}$ & 3.808211 & -2.010887 & -0.264371 \\
$\mathrm{Pd}$ & -0.669061 & -0.184997 & -0.181217 \\
$\mathrm{O}$ & -2.574466 & 0.782954 & -0.096348 \\
$\mathrm{C}$ & -3.609607 & 0.109712 & 0.232888 \\
$\mathrm{O}$ & -4.733805 & 0.881822 & 0.341465 \\
$\mathrm{C}$ & 1.507701 & -0.237502 & 2.212319 \\
$\mathrm{C}$ & 1.824011 & 1.291414 & 2.185832 \\
$\mathrm{C}$ & 1.438729 & 1.928204 & 0.872639 \\
$\mathrm{C}$ & 0.076995 & 2.064289 & 0.512259 \\
$\mathrm{C}$ & -0.283787 & 2.531766 & -0.767525 \\
$\mathrm{C}$ & 0.708253 & 2.885280 & -1.688896 \\
$\mathrm{C}$ & 2.048820 & 2.782957 & -1.323410 \\
$\mathrm{C}$ & 2.408236 & 2.298769 & -0.060173 \\
$\mathrm{P}$ & -1.475215 & -2.321196 & -0.322609 \\
$\mathrm{O}$ & -3.696257 & -1.111737 & 0.451529 \\
$\mathrm{H}$ & -1.909806 & -2.943359 & 0.856348 \\
$\mathrm{H}$ & 1.108471 & -1.580976 & -2.279556 \\
$\mathrm{H}$ & -0.528976 & -3.277740 & -0.762123 \\
$\mathrm{H}$ & -2.521317 & -2.590171 & -1.217152 \\
$\mathrm{H}$ & -1.336980 & 2.632193 & -1.005811 \\
$\mathrm{H}$ & 0.431554 & 3.249750 & -2.673915 \\
$\mathrm{H}$ & 2.826692 & 3.065369 & -2.028170 \\
$\mathrm{H}$ & 3.459484 & 2.193239 & 0.194493 \\
$\mathrm{H}$ & 3.904095 & -1.432074 & 1.807284 \\
$\mathrm{H}$ & 4.817096 & -2.415058 & -0.276879 \\
$\mathrm{H}$ & 3.414464 & -2.458065 & -2.339681 \\
$\mathrm{H}$ & -0.701818 & 1.930995 & 1.260845 \\
$\mathrm{H}$ & 1.977296 & -0.671879 & 3.103474 \\
$\mathrm{H}$ & 0.424649 & -0.368825 & 2.323094 \\
$\mathrm{H}$ & 2.897307 & 1.434560 & 2.355384 \\
$\mathrm{H}$ & 1.297023 & 1.771626 & 3.020783 \\
$\mathrm{H}$ & -5.439287 & 0.260720 & 0.588978 \\
\hline \hline & & &
\end{tabular}


Table S5: Cartesian coordinates $(\AA)$ of t1aTS for the intramolecular assisted mechanism $(\mathrm{E}=$ -1275.59076206 a.u.).

\begin{tabular}{lrrr}
\hline \hline $\mathrm{C}$ & 3.712595 & -1.086930 & -1.063725 \\
$\mathrm{C}$ & 2.317507 & -1.108486 & -1.186954 \\
$\mathrm{C}$ & 1.502486 & -0.715276 & -0.119081 \\
$\mathrm{C}$ & 2.087779 & -0.275327 & 1.081443 \\
$\mathrm{C}$ & 3.484829 & -0.268489 & 1.192804 \\
$\mathrm{C}$ & 4.297721 & -0.674608 & 0.132375 \\
$\mathrm{Pd}$ & -0.514016 & -0.789364 & -0.250806 \\
$\mathrm{O}$ & -2.740931 & -0.897357 & -0.304412 \\
$\mathrm{C}$ & -3.317533 & -0.089942 & 0.450414 \\
$\mathrm{O}$ & -4.634219 & -0.272573 & 0.670938 \\
$\mathrm{C}$ & 1.218644 & 0.270585 & 2.193364 \\
$\mathrm{C}$ & 0.860592 & 1.762806 & 1.975972 \\
$\mathrm{C}$ & 0.430903 & 2.158673 & 0.572361 \\
$\mathrm{C}$ & -0.502499 & 1.396387 & -0.179694 \\
$\mathrm{C}$ & -0.881440 & 1.883790 & -1.453559 \\
$\mathrm{C}$ & -0.362177 & 3.063655 & -1.983537 \\
$\mathrm{C}$ & 0.556350 & 3.795751 & -1.229572 \\
$\mathrm{C}$ & 0.938746 & 3.348260 & 0.036742 \\
$\mathrm{P}$ & -0.355631 & -3.117870 & -0.373995 \\
$\mathrm{O}$ & -2.781034 & 0.916646 & 1.049682 \\
$\mathrm{H}$ & 0.296411 & -3.804728 & 0.673104 \\
$\mathrm{H}$ & 1.873863 & -1.429568 & -2.126481 \\
$\mathrm{H}$ & 0.332025 & -3.716097 & -1.453411 \\
$\mathrm{H}$ & -1.544645 & -3.880362 & -0.424920 \\
$\mathrm{H}$ & -1.620020 & 1.328494 & -2.028967 \\
$\mathrm{H}$ & -0.669659 & 3.411335 & -2.966582 \\
$\mathrm{H}$ & 0.974021 & 4.719469 & -1.623127 \\
$\mathrm{H}$ & 1.650132 & 3.933667 & 0.615895 \\
$\mathrm{H}$ & 3.939782 & 0.066520 & 2.123738 \\
$\mathrm{H}$ & 5.379249 & -0.664401 & 0.240472 \\
$\mathrm{H}$ & 4.333279 & -1.395392 & -1.901788 \\
$\mathrm{H}$ & -1.669232 & 1.053370 & 0.564223 \\
$\mathrm{H}$ & 1.725997 & 0.178546 & 3.161922 \\
$\mathrm{H}$ & 0.296562 & -0.317372 & 2.260225 \\
$\mathrm{H}$ & 1.727716 & 2.374652 & 2.253014 \\
$\mathrm{H}$ & 0.061049 & 2.028517 & 2.683880 \\
$\mathrm{H}$ & -4.924914 & 0.453319 & 1.250008 \\
\hline \hline & & &
\end{tabular}


Table S6: Cartesian coordinates $(\AA)$ of $\mathbf{t 1 a R}$ for the intermolecular assisted mechanism $(\mathrm{E}=$ -1288.89630758 a.u.).

\begin{tabular}{lrrr}
\hline \hline $\mathrm{C}$ & 3.422797 & -0.541865 & -2.421887 \\
$\mathrm{C}$ & 2.105128 & -0.176070 & -2.123127 \\
$\mathrm{C}$ & 1.513837 & -0.521383 & -0.898388 \\
$\mathrm{C}$ & 2.246826 & -1.282267 & 0.037698 \\
$\mathrm{C}$ & 3.564637 & -1.645821 & -0.285136 \\
$\mathrm{C}$ & 4.155701 & -1.284765 & -1.497183 \\
$\mathrm{Pd}$ & -0.351311 & 0.253174 & -0.610579 \\
$\mathrm{C}$ & 1.663359 & -1.662966 & 1.379058 \\
$\mathrm{C}$ & 1.853551 & -0.568538 & 2.476981 \\
$\mathrm{C}$ & 1.581395 & 0.825558 & 1.969625 \\
$\mathrm{C}$ & 0.257514 & 1.222378 & 1.664550 \\
$\mathrm{C}$ & 0.016384 & 2.476574 & 1.076356 \\
$\mathrm{C}$ & 1.082606 & 3.347725 & 0.799843 \\
$\mathrm{C}$ & 2.379781 & 2.968528 & 1.127929 \\
$\mathrm{C}$ & 2.623608 & 1.714042 & 1.703674 \\
$\mathrm{P}$ & -1.128800 & -1.609821 & -1.632233 \\
$\mathrm{H}$ & -2.471176 & -1.936292 & -1.424993 \\
$\mathrm{H}$ & 1.533807 & 0.389203 & -2.857343 \\
$\mathrm{H}$ & -0.455804 & -2.834956 & -1.535905 \\
$\mathrm{H}$ & -1.123620 & -1.465869 & -3.048846 \\
$\mathrm{H}$ & 3.645665 & 1.416640 & 1.929617 \\
$\mathrm{H}$ & 4.134160 & -2.231513 & 0.436176 \\
$\mathrm{H}$ & 5.176601 & -1.589588 & -1.719189 \\
$\mathrm{H}$ & 3.863131 & -0.255304 & -3.375325 \\
$\mathrm{H}$ & -0.563757 & 0.567409 & 1.966547 \\
$\mathrm{H}$ & 2.143391 & -2.582044 & 1.742801 \\
$\mathrm{H}$ & 0.594636 & -1.880218 & 1.274247 \\
$\mathrm{H}$ & 2.879835 & -0.612415 & 2.866528 \\
$\mathrm{H}$ & 1.164579 & -0.814928 & 3.292871 \\
$\mathrm{Br}$ & -2.708954 & 1.385977 & -0.926648 \\
$\mathrm{O}$ & -1.593097 & -1.248936 & 2.620201 \\
$\mathrm{C}$ & -2.003365 & -1.995856 & 1.703475 \\
$\mathrm{O}$ & -1.364674 & -2.592147 & 0.796435 \\
$\mathrm{O}$ & -3.388527 & -2.222033 & 1.665834 \\
$\mathrm{H}$ & -3.723020 & -1.653022 & 2.378597 \\
$\mathrm{H}$ & -1.005412 & 2.773222 & 0.863944 \\
$\mathrm{H}$ & 3.213028 & 3.639159 & 0.927267 \\
$\mathrm{H}$ & 0.887481 & 4.315901 & 0.345259 \\
\hline \hline & & &
\end{tabular}


Table S7: Cartesian coordinates $(\AA)$ of t1aTS for the intermolecular assisted mechanism $(\mathrm{E}=$ -1288.86865585 a.u.).

\begin{tabular}{lrrr}
\hline \hline $\mathrm{C}$ & 3.528587 & -2.436604 & -0.528732 \\
$\mathrm{C}$ & 2.190738 & -2.070400 & -0.719613 \\
$\mathrm{C}$ & 1.581240 & -1.101843 & 0.090863 \\
$\mathrm{C}$ & 2.337884 & -0.476323 & 1.097891 \\
$\mathrm{C}$ & 3.673985 & -0.860549 & 1.286561 \\
$\mathrm{C}$ & 4.272180 & -1.836078 & 0.486781 \\
$\mathrm{Pd}$ & -0.385383 & -0.667515 & -0.229216 \\
$\mathrm{C}$ & 1.744796 & 0.661451 & 1.892782 \\
$\mathrm{C}$ & 1.842184 & 2.008423 & 1.131371 \\
$\mathrm{C}$ & 1.434743 & 1.979701 & -0.329641 \\
$\mathrm{C}$ & 0.189135 & 1.418302 & -0.735977 \\
$\mathrm{C}$ & -0.182998 & 1.552912 & -2.093736 \\
$\mathrm{C}$ & 0.647188 & 2.172083 & -3.031433 \\
$\mathrm{C}$ & 1.873563 & 2.690478 & -2.617379 \\
$\mathrm{C}$ & 2.253943 & 2.603899 & -1.274188 \\
$\mathrm{P}$ & -0.999827 & -2.791277 & 0.452874 \\
$\mathrm{H}$ & -2.032777 & -2.863140 & 1.409330 \\
$\mathrm{H}$ & 1.622817 & -2.548819 & -1.515474 \\
$\mathrm{H}$ & -0.074962 & -3.669472 & 1.073772 \\
$\mathrm{H}$ & -1.526880 & -3.704282 & -0.489595 \\
$\mathrm{H}$ & 3.203087 & 3.034352 & -0.958080 \\
$\mathrm{H}$ & 4.253497 & -0.377338 & 2.072749 \\
$\mathrm{H}$ & 5.309024 & -2.120669 & 0.654227 \\
$\mathrm{H}$ & 3.980312 & -3.191603 & -1.170040 \\
$\mathrm{H}$ & -0.724278 & 1.684650 & 0.210042 \\
$\mathrm{H}$ & 2.259830 & 0.775342 & 2.855137 \\
$\mathrm{H}$ & 0.698083 & 0.450281 & 2.122125 \\
$\mathrm{H}$ & 2.873273 & 2.380662 & 1.199155 \\
$\mathrm{H}$ & 1.194231 & 2.724954 & 1.653514 \\
$\mathrm{Br}$ & -2.983421 & -0.528853 & -0.917640 \\
$\mathrm{O}$ & -1.341605 & 2.397885 & 1.109596 \\
$\mathrm{C}$ & -1.544306 & 1.842572 & 2.272828 \\
$\mathrm{O}$ & -1.167682 & 2.257133 & 3.360976 \\
$\mathrm{O}$ & -2.255066 & 0.658133 & 2.238677 \\
$\mathrm{H}$ & -2.552240 & 0.513476 & 1.316840 \\
$\mathrm{H}$ & -1.152748 & 1.172819 & -2.403842 \\
$\mathrm{H}$ & 2.533862 & 3.175973 & -3.334205 \\
$\mathrm{H}$ & 0.338284 & 2.254169 & -4.071701 \\
\hline \hline & & &
\end{tabular}


Table S8: Cartesian coordinates $(\AA)$ of $\mathbf{t 1} \mathbf{b R}$ for the unassisted mechanism $(E=-1322.10820550$ a.u.).

\begin{tabular}{lrrr}
\hline \hline $\mathrm{C}$ & 2.893261 & -2.216995 & -1.682543 \\
$\mathrm{C}$ & 1.626258 & -1.628851 & -1.586427 \\
$\mathrm{C}$ & 1.032842 & -1.416036 & -0.337538 \\
$\mathrm{C}$ & 1.708350 & -1.810217 & 0.834259 \\
$\mathrm{C}$ & 2.971052 & -2.409832 & 0.721041 \\
$\mathrm{C}$ & 3.562773 & -2.617313 & -0.526466 \\
$\mathrm{Pd}$ & -0.801839 & -0.543659 & -0.116716 \\
$\mathrm{C}$ & 1.132209 & -1.481534 & 2.195555 \\
$\mathrm{C}$ & 1.460872 & -0.020340 & 2.638930 \\
$\mathrm{C}$ & 1.276081 & 0.995392 & 1.535294 \\
$\mathrm{C}$ & -0.013113 & 1.458775 & 1.170196 \\
$\mathrm{C}$ & -0.136798 & 2.395245 & 0.126146 \\
$\mathrm{C}$ & 0.974241 & 2.819242 & -0.597967 \\
$\mathrm{C}$ & 2.229605 & 2.340163 & -0.231703 \\
$\mathrm{C}$ & 2.387970 & 1.443412 & 0.817797 \\
$\mathrm{P}$ & -1.771111 & -2.323330 & -1.103839 \\
$\mathrm{H}$ & -2.559463 & -3.135205 & -0.268629 \\
$\mathrm{H}$ & 1.111052 & -1.332547 & -2.497682 \\
$\mathrm{H}$ & -0.976781 & -3.306831 & -1.734061 \\
$\mathrm{H}$ & -2.688389 & -2.038373 & -2.130382 \\
$\mathrm{H}$ & 3.386656 & 1.090670 & 1.050235 \\
$\mathrm{H}$ & 3.495396 & -2.716075 & 1.624992 \\
$\mathrm{H}$ & 4.539147 & -3.089806 & -0.593444 \\
$\mathrm{H}$ & 3.346625 & -2.366952 & -2.659411 \\
$\mathrm{H}$ & -0.881738 & 1.279772 & 1.797906 \\
$\mathrm{H}$ & 1.519612 & -2.165973 & 2.959829 \\
$\mathrm{H}$ & 0.041706 & -1.602235 & 2.179358 \\
$\mathrm{H}$ & 2.502877 & 0.017613 & 2.974641 \\
$\mathrm{H}$ & 0.833297 & 0.234936 & 3.501401 \\
$\mathrm{Br}$ & -3.265127 & 0.205397 & 0.267490 \\
$\mathrm{~F}$ & -1.313094 & 2.945387 & -0.161295 \\
$\mathrm{~F}$ & 0.843660 & 3.699304 & -1.596129 \\
$\mathrm{~F}$ & 3.294669 & 2.765719 & -0.920337 \\
\hline \hline & & &
\end{tabular}


Table S9: Cartesian coordinates $(\AA)$ of t1bTS for the unassisted mechanism $(\mathrm{E}=$ 1322.05320211 a.u.).

\begin{tabular}{lrrr}
\hline \hline $\mathrm{C}$ & 2.276588 & 3.228103 & -1.479433 \\
$\mathrm{C}$ & 1.865839 & 1.888451 & -1.492297 \\
$\mathrm{C}$ & 1.512772 & 1.259335 & -0.297070 \\
$\mathrm{C}$ & 1.534659 & 1.959527 & 0.919951 \\
$\mathrm{C}$ & 1.952629 & 3.297255 & 0.911700 \\
$\mathrm{C}$ & 2.324482 & 3.930813 & -0.276351 \\
$\mathrm{Pd}$ & 0.974177 & -0.686133 & -0.210762 \\
$\mathrm{C}$ & 1.074946 & 1.286439 & 2.197279 \\
$\mathrm{C}$ & -0.458880 & 1.300416 & 2.399218 \\
$\mathrm{C}$ & -1.340124 & 0.961425 & 1.203521 \\
$\mathrm{C}$ & -0.955552 & 0.072583 & 0.169113 \\
$\mathrm{C}$ & -1.857045 & -0.116847 & -0.883311 \\
$\mathrm{C}$ & -3.112580 & 0.476229 & -0.936313 \\
$\mathrm{C}$ & -3.472670 & 1.325273 & 0.105394 \\
$\mathrm{C}$ & -2.601574 & 1.571323 & 1.158540 \\
$\mathrm{P}$ & 3.182399 & -1.372784 & -0.728982 \\
$\mathrm{H}$ & 4.230300 & -0.924665 & 0.104941 \\
$\mathrm{H}$ & 1.822960 & 1.350021 & -2.435207 \\
$\mathrm{H}$ & 3.736336 & -0.968710 & -1.964072 \\
$\mathrm{H}$ & 3.543524 & -2.739343 & -0.785240 \\
$\mathrm{H}$ & -2.921706 & 2.258487 & 1.936307 \\
$\mathrm{H}$ & 1.982918 & 3.847986 & 1.850288 \\
$\mathrm{H}$ & 2.646497 & 4.968627 & -0.260561 \\
$\mathrm{H}$ & 2.556679 & 3.713762 & -2.411023 \\
$\mathrm{H}$ & 1.526684 & 1.778811 & 3.067379 \\
$\mathrm{H}$ & 1.430935 & 0.249702 & 2.204202 \\
$\mathrm{H}$ & -0.753065 & 2.297126 & 2.749408 \\
$\mathrm{H}$ & -0.696154 & 0.612192 & 3.224284 \\
$\mathrm{Br}$ & -0.340925 & -3.098170 & 0.705260 \\
$\mathrm{H}$ & -0.804263 & -1.604687 & 0.663761 \\
$\mathrm{~F}$ & -1.525570 & -0.933519 & -1.908107 \\
$\mathrm{~F}$ & -3.948886 & 0.254102 & -1.961026 \\
$\mathrm{~F}$ & -4.678693 & 1.913699 & 0.069305 \\
\hline \hline & & &
\end{tabular}


Table S10: Cartesian coordinates $(\AA)$ of $\mathbf{t 1} \mathbf{b R}$ for the intramolecular assisted mechanism $(\mathrm{E}=$ -1573.30322711 a.u.).

\begin{tabular}{lrrr}
\hline \hline $\mathrm{C}$ & 3.276131 & -1.988970 & -1.310447 \\
$\mathrm{C}$ & 1.962315 & -1.517449 & -1.422265 \\
$\mathrm{C}$ & 1.167096 & -1.338910 & -0.283230 \\
$\mathrm{C}$ & 1.693104 & -1.639651 & 0.989716 \\
$\mathrm{C}$ & 3.005615 & -2.123420 & 1.082784 \\
$\mathrm{C}$ & 3.794235 & -2.304710 & -0.055445 \\
$\mathrm{Pd}$ & -0.740149 & -0.645227 & -0.377482 \\
$\mathrm{O}$ & -2.791509 & -0.057187 & -0.525145 \\
$\mathrm{C}$ & -3.376397 & -0.084274 & 0.615051 \\
$\mathrm{O}$ & -4.719094 & 0.161271 & 0.531094 \\
$\mathrm{C}$ & 0.906634 & -1.315071 & 2.242210 \\
$\mathrm{C}$ & 1.109094 & 0.170117 & 2.674546 \\
$\mathrm{C}$ & 0.942252 & 1.149004 & 1.534914 \\
$\mathrm{C}$ & -0.343051 & 1.407980 & 0.997225 \\
$\mathrm{C}$ & -0.470636 & 2.314007 & -0.068267 \\
$\mathrm{C}$ & 0.645669 & 2.917274 & -0.643953 \\
$\mathrm{C}$ & 1.899909 & 2.636241 & -0.109021 \\
$\mathrm{C}$ & 2.056937 & 1.766713 & 0.965770 \\
$\mathrm{P}$ & -1.242937 & -2.547842 & -1.480831 \\
$\mathrm{O}$ & -2.860840 & -0.296946 & 1.727340 \\
$\mathrm{H}$ & -1.074445 & -3.777689 & -0.813267 \\
$\mathrm{H}$ & 1.569002 & -1.281197 & -2.408511 \\
$\mathrm{H}$ & -0.575546 & -2.853421 & -2.684663 \\
$\mathrm{H}$ & -2.587321 & -2.651610 & -1.887266 \\
$\mathrm{H}$ & 3.058975 & 1.574900 & 1.333836 \\
$\mathrm{H}$ & 3.414370 & -2.356575 & 2.064748 \\
$\mathrm{H}$ & 4.806845 & -2.687644 & 0.039945 \\
$\mathrm{H}$ & 3.883513 & -2.113844 & -2.203556 \\
$\mathrm{H}$ & -1.242356 & 1.054136 & 1.504062 \\
$\mathrm{H}$ & 1.217158 & -1.958419 & 3.074383 \\
$\mathrm{H}$ & -0.165222 & -1.484719 & 2.084983 \\
$\mathrm{H}$ & 2.115316 & 0.283777 & 3.093256 \\
$\mathrm{H}$ & 0.393796 & 0.398499 & 3.473818 \\
$\mathrm{H}$ & -5.033394 & 0.130864 & 1.450624 \\
$\mathrm{~F}$ & -1.674911 & 2.656980 & -0.526040 \\
$\mathrm{~F}$ & 0.513417 & 3.770217 & -1.666570 \\
$\mathrm{~F}$ & 2.969086 & 3.225097 & -0.659570 \\
\hline \hline & & &
\end{tabular}


Table S11: Cartesian coordinates $(\AA)$ of t1bTS for the intramolecular assisted mechanism $(\mathrm{E}=$ -1573.28220426 a.u.).

\begin{tabular}{lrrr}
\hline \hline $\mathrm{C}$ & -1.520147 & 3.501288 & -1.349909 \\
$\mathrm{C}$ & -0.605087 & 2.442046 & -1.407466 \\
$\mathrm{C}$ & -0.155156 & 1.829591 & -0.232863 \\
$\mathrm{C}$ & -0.633360 & 2.268483 & 1.015449 \\
$\mathrm{C}$ & -1.541574 & 3.334569 & 1.056218 \\
$\mathrm{C}$ & -1.982662 & 3.953956 & -0.115358 \\
$\mathrm{Pd}$ & 1.188975 & 0.320027 & -0.252798 \\
$\mathrm{O}$ & 2.741402 & -1.247967 & -0.167225 \\
$\mathrm{C}$ & 2.582333 & -2.153235 & 0.676930 \\
$\mathrm{O}$ & 3.596840 & -3.025462 & 0.841738 \\
$\mathrm{C}$ & -0.237054 & 1.534729 & 2.279394 \\
$\mathrm{C}$ & -1.111607 & 0.275985 & 2.539230 \\
$\mathrm{C}$ & -1.461566 & -0.548231 & 1.312711 \\
$\mathrm{C}$ & -0.458283 & -1.001074 & 0.409030 \\
$\mathrm{C}$ & -0.891219 & -1.765555 & -0.682723 \\
$\mathrm{C}$ & -2.220492 & -2.095804 & -0.915328 \\
$\mathrm{C}$ & -3.169438 & -1.634480 & -0.008612 \\
$\mathrm{C}$ & -2.802494 & -0.872712 & 1.097174 \\
$\mathrm{P}$ & 2.769505 & 1.846626 & -0.995961 \\
$\mathrm{O}$ & 1.549246 & -2.343416 & 1.418143 \\
$\mathrm{H}$ & 3.019354 & 2.998969 & -0.221022 \\
$\mathrm{H}$ & -0.252932 & 2.097377 & -2.376895 \\
$\mathrm{H}$ & 2.578767 & 2.479169 & -2.243314 \\
$\mathrm{H}$ & 4.095799 & 1.393876 & -1.167360 \\
$\mathrm{H}$ & -3.585249 & -0.537031 & 1.770348 \\
$\mathrm{H}$ & -1.910329 & 3.679425 & 2.020944 \\
$\mathrm{H}$ & -2.684755 & 4.781819 & -0.061851 \\
$\mathrm{H}$ & -1.862843 & 3.968451 & -2.270055 \\
$\mathrm{H}$ & 0.638904 & -1.607914 & 0.984966 \\
$\mathrm{H}$ & -0.321825 & 2.194013 & 3.152068 \\
$\mathrm{H}$ & 0.814477 & 1.233178 & 2.212601 \\
$\mathrm{H}$ & -2.047099 & 0.589623 & 3.016062 \\
$\mathrm{H}$ & -0.590016 & -0.361169 & 3.267424 \\
$\mathrm{H}$ & 3.303238 & -3.672676 & 1.506135 \\
$\mathrm{~F}$ & 0.013385 & -2.243746 & -1.561753 \\
$\mathrm{~F}$ & -2.587882 & -2.830747 & -1.975483 \\
$\mathrm{~F}$ & -4.457059 & -1.941098 & -0.219034 \\
\hline \hline & & &
\end{tabular}


Table S12: Cartesian coordinates $(\AA)$ of $\mathbf{t 1} \mathbf{b R}$ for the intermolecular assisted mechanism $(\mathrm{E}=$ -1586.58181355 a.u.).

\begin{tabular}{lrrr}
\hline \hline $\mathrm{C}$ & 3.941181 & 1.874078 & -1.540331 \\
$\mathrm{C}$ & 2.944081 & 0.896503 & -1.593405 \\
$\mathrm{C}$ & 2.203530 & 0.548253 & -0.450372 \\
$\mathrm{C}$ & 2.508937 & 1.145381 & 0.790080 \\
$\mathrm{C}$ & 3.518967 & 2.124954 & 0.818671 \\
$\mathrm{C}$ & 4.227423 & 2.494962 & -0.323601 \\
$\mathrm{Pd}$ & 0.562956 & -0.555672 & -0.862805 \\
$\mathrm{C}$ & 1.807046 & 0.788788 & 2.084789 \\
$\mathrm{C}$ & 0.687755 & 1.777010 & 2.505878 \\
$\mathrm{C}$ & -0.544489 & 1.802049 & 1.625336 \\
$\mathrm{C}$ & -1.479529 & 0.753278 & 1.679544 \\
$\mathrm{C}$ & -2.640312 & 0.837953 & 0.921272 \\
$\mathrm{C}$ & -2.895438 & 1.930433 & 0.093234 \\
$\mathrm{C}$ & -1.953050 & 2.949177 & 0.029812 \\
$\mathrm{C}$ & -0.788698 & 2.898450 & 0.785836 \\
$\mathrm{P}$ & 1.804093 & -2.364868 & -0.389871 \\
$\mathrm{H}$ & 1.143362 & -3.587605 & -0.232223 \\
$\mathrm{H}$ & 2.730309 & 0.407067 & -2.541678 \\
$\mathrm{H}$ & 2.909299 & -2.375733 & 0.468236 \\
$\mathrm{H}$ & 2.508832 & -2.645429 & -1.603005 \\
$\mathrm{H}$ & -0.085377 & 3.722513 & 0.717217 \\
$\mathrm{H}$ & 3.757459 & 2.596416 & 1.771855 \\
$\mathrm{H}$ & 5.008457 & 3.249875 & -0.260395 \\
$\mathrm{H}$ & 4.492955 & 2.138504 & -2.440286 \\
$\mathrm{H}$ & -1.317652 & -0.135642 & 2.291996 \\
$\mathrm{H}$ & 2.552489 & 0.799493 & 2.893138 \\
$\mathrm{H}$ & 1.402898 & -0.228179 & 2.044625 \\
$\mathrm{H}$ & 1.103410 & 2.791984 & 2.565320 \\
$\mathrm{H}$ & 0.381408 & 1.499984 & 3.522982 \\
$\mathrm{Br}$ & -1.535728 & -1.415605 & -2.017646 \\
$\mathrm{O}$ & -1.035661 & -2.204168 & 2.937286 \\
$\mathrm{C}$ & -0.179044 & -2.699933 & 2.189679 \\
$\mathrm{O}$ & 0.966821 & -2.260575 & 1.868079 \\
$\mathrm{O}$ & -0.481176 & -3.934840 & 1.598359 \\
$\mathrm{H}$ & -1.408671 & -4.085743 & 1.844792 \\
$\mathrm{~F}$ & -3.566382 & -0.130310 & 0.977277 \\
$\mathrm{~F}$ & -4.028423 & 2.000814 & -0.626658 \\
$\mathrm{~F}$ & -2.198096 & 4.007414 & -0.774640 \\
\hline \hline & & &
\end{tabular}


Table S13: Cartesian coordinates $(\AA)$ of $\mathbf{t 1 b T S}$ for the intermolecular assisted mechanism $(\mathrm{E}=$ -1586.55886914 a.u.).

\begin{tabular}{lrrr}
\hline \hline $\mathrm{C}$ & -2.265304 & 3.288470 & -1.650531 \\
$\mathrm{C}$ & -1.271094 & 2.302283 & -1.660480 \\
$\mathrm{C}$ & -0.740990 & 1.802735 & -0.463661 \\
$\mathrm{C}$ & -1.225434 & 2.289443 & 0.763641 \\
$\mathrm{C}$ & -2.213011 & 3.285453 & 0.759020 \\
$\mathrm{C}$ & -2.730939 & 3.790383 & -0.435505 \\
$\mathrm{Pd}$ & 0.755306 & 0.420443 & -0.524882 \\
$\mathrm{C}$ & -0.764218 & 1.658003 & 2.056321 \\
$\mathrm{C}$ & -1.554761 & 0.358485 & 2.367953 \\
$\mathrm{C}$ & -1.780387 & -0.566572 & 1.187640 \\
$\mathrm{C}$ & -0.678549 & -1.063919 & 0.423191 \\
$\mathrm{C}$ & -0.985413 & -2.001200 & -0.573495 \\
$\mathrm{C}$ & -2.285831 & -2.409066 & -0.866688 \\
$\mathrm{C}$ & -3.326908 & -1.880308 & -0.118262 \\
$\mathrm{C}$ & -3.085589 & -0.972076 & 0.907619 \\
$\mathrm{P}$ & 2.199084 & 2.026996 & -1.309531 \\
$\mathrm{H}$ & 3.378859 & 2.220011 & -0.565466 \\
$\mathrm{H}$ & -0.912154 & 1.923832 & -2.615763 \\
$\mathrm{H}$ & 1.792178 & 3.381317 & -1.428842 \\
$\mathrm{H}$ & 2.764376 & 1.869058 & -2.592452 \\
$\mathrm{H}$ & -3.931130 & -0.598649 & 1.477477 \\
$\mathrm{H}$ & -2.584014 & 3.663243 & 1.711243 \\
$\mathrm{H}$ & -3.493854 & 4.565773 & -0.415642 \\
$\mathrm{H}$ & -2.665844 & 3.662918 & -2.590865 \\
$\mathrm{H}$ & 0.340578 & -1.281089 & 1.208478 \\
$\mathrm{H}$ & -0.895406 & 2.346445 & 2.900252 \\
$\mathrm{H}$ & 0.303173 & 1.427663 & 2.003699 \\
$\mathrm{H}$ & -2.534960 & 0.633975 & 2.777364 \\
$\mathrm{H}$ & -1.006640 & -0.184494 & 3.148606 \\
$\mathrm{Br}$ & 2.949663 & -1.075396 & -0.983313 \\
$\mathrm{O}$ & 0.982865 & -1.656542 & 2.314099 \\
$\mathrm{C}$ & 1.603149 & -0.720886 & 2.973008 \\
$\mathrm{O}$ & 1.345743 & -0.313718 & 4.100848 \\
$\mathrm{O}$ & 2.649196 & -0.124202 & 2.295486 \\
$\mathrm{H}$ & 2.763636 & -0.604305 & 1.448980 \\
$\mathrm{~F}$ & -0.020280 & -2.577407 & -1.307217 \\
$\mathrm{~F}$ & -2.535016 & -3.298798 & -1.848821 \\
$\mathrm{~F}$ & -4.586717 & -2.273111 & -0.395705 \\
\hline \hline & & & \\
\hline
\end{tabular}


Table S14: Cartesian coordinates $(\AA)$ of t2aR $(\mathrm{E}=$ 1288.734358237030 a.u.).

\begin{tabular}{lrrr}
\hline \hline $\mathrm{C}$ & -0.797926 & -2.903901 & 2.744810 \\
$\mathrm{C}$ & -0.619781 & -2.203832 & 1.547142 \\
$\mathrm{C}$ & 0.589005 & -1.567816 & 1.242604 \\
$\mathrm{C}$ & 1.651011 & -1.623621 & 2.168520 \\
$\mathrm{C}$ & 1.450925 & -2.321553 & 3.371657 \\
$\mathrm{C}$ & 0.244660 & -2.956783 & 3.667907 \\
$\mathrm{Pd}$ & 0.755028 & -0.520090 & -0.505153 \\
$\mathrm{C}$ & 3.001293 & -1.017466 & 1.874781 \\
$\mathrm{C}$ & 3.900739 & -1.981320 & 1.048354 \\
$\mathrm{C}$ & 3.171212 & -2.573740 & -0.124053 \\
$\mathrm{C}$ & 2.771622 & -1.732106 & -1.197105 \\
$\mathrm{C}$ & 2.069051 & -2.278873 & -2.292454 \\
$\mathrm{C}$ & 1.760168 & -3.643299 & -2.329791 \\
$\mathrm{C}$ & 2.154977 & -4.463417 & -1.274927 \\
$\mathrm{C}$ & 2.846991 & -3.930811 & -0.181904 \\
$\mathrm{H}$ & -1.450931 & -2.137280 & 0.859118 \\
$\mathrm{H}$ & 3.143336 & -4.582058 & 0.637400 \\
$\mathrm{H}$ & 2.268888 & -2.356263 & 4.089838 \\
$\mathrm{H}$ & 0.119993 & -3.479796 & 4.613841 \\
$\mathrm{H}$ & -1.751576 & -3.385867 & 2.952222 \\
$\mathrm{H}$ & 3.217380 & -0.732697 & -1.247015 \\
$\mathrm{H}$ & 3.529557 & -0.768077 & 2.800743 \\
$\mathrm{H}$ & 2.928653 & -0.076797 & 1.324280 \\
$\mathrm{H}$ & 4.268092 & -2.788997 & 1.695676 \\
$\mathrm{H}$ & 4.737461 & -1.374728 & 0.677477 \\
$\mathrm{Br}$ & 0.852633 & 0.984081 & -2.742425 \\
$\mathrm{O}$ & 5.100053 & 0.286191 & -0.705967 \\
$\mathrm{C}$ & 5.285408 & 1.224502 & 0.124084 \\
$\mathrm{C}$ & 4.929678 & 1.348287 & 1.306366 \\
$\mathrm{H}$ & -1.183637 & 3.815669 & 1.470939 \\
$\mathrm{O}$ & 6.036657 & 2.308872 & -0.399122 \\
$\mathrm{H}$ & 6.185320 & 2.026930 & -1.316499 \\
$\mathrm{P}$ & -0.645199 & 1.171449 & 0.227330 \\
$\mathrm{C}$ & -1.938423 & 1.645056 & -1.002364 \\
$\mathrm{C}$ & 0.391789 & 2.644300 & 0.537121 \\
$\mathrm{C}$ & -1.474895 & 1.035450 & 1.856207 \\
$\mathrm{C}$ & -2.834406 & 0.688630 & -1.570151 \\
$\mathrm{C}$ & -2.023443 & 2.986297 & -1.440942 \\
$\mathrm{C}$ & -0.141012 & 3.779036 & 1.182576 \\
$\mathrm{C}$ & 1.704405 & 2.619348 & 0.221384 \\
$\mathrm{C}$ & -0.724780 & 0.809073 & 2.955969 \\
$\mathrm{C}$ & -2.846786 & 1.316308 & 1.997768 \\
$\mathrm{C}$ & -3.734623 & 1.114636 & -2.561380 \\
$\mathrm{H}$ & -2.955666 & -0.775750 & -1.327551 \\
$\mathrm{H}$ & -1.350243 & 3.739695 & -1.071853 \\
$\mathrm{C}$ & & 3.680566 & 0.471297
\end{tabular}




\begin{tabular}{rrrr}
$\mathrm{H}$ & 2.138206 & 1.739613 & -0.234670 \\
$\mathrm{H}$ & 0.344052 & 0.674451 & 2.868322 \\
$\mathrm{C}$ & -1.298566 & 0.780040 & 4.232699 \\
$\mathrm{C}$ & -3.436398 & 1.324776 & 3.266712 \\
$\mathrm{H}$ & -3.459265 & 1.557978 & 1.141729 \\
$\mathrm{C}$ & -3.796684 & 2.454560 & -2.931187 \\
$\mathrm{H}$ & -4.366931 & 0.402750 & -3.076093 \\
$\mathrm{C}$ & -3.727516 & -1.477343 & -0.556966 \\
$\mathrm{C}$ & -1.833212 & -1.484527 & -2.016847 \\
$\mathrm{H}$ & -2.962612 & 4.410189 & -2.666896 \\
$\mathrm{C}$ & 1.980313 & 4.830260 & 1.088622 \\
$\mathrm{H}$ & 0.284005 & 5.758503 & 1.925820 \\
$\mathrm{H}$ & 3.544137 & 3.647725 & 0.199716 \\
$\mathrm{C}$ & -2.661267 & 1.036863 & 4.389866 \\
$\mathrm{H}$ & -0.680944 & 0.582978 & 5.099341 \\
$\mathrm{H}$ & -4.487622 & 1.555966 & 3.378002 \\
$\mathrm{H}$ & -4.499052 & 2.775623 & -3.689586 \\
$\mathrm{C}$ & -3.678595 & -2.883912 & -0.524743 \\
$\mathrm{~N}$ & -4.747546 & -0.796370 & 0.199199 \\
$\mathrm{C}$ & -1.799793 & -2.884779 & -1.967899 \\
$\mathrm{H}$ & -1.129874 & -0.983030 & -2.663325 \\
$\mathrm{H}$ & 2.627713 & 5.675205 & 1.285628 \\
$\mathrm{H}$ & -3.107969 & 1.032659 & 5.375573 \\
$\mathrm{C}$ & -2.696151 & -3.566392 & -1.243344 \\
$\mathrm{H}$ & -4.403804 & -3.449693 & 0.045118 \\
$\mathrm{C}$ & -4.948272 & -1.165949 & 1.601863 \\
$\mathrm{C}$ & -5.867376 & -0.162269 & -0.497806 \\
$\mathrm{H}$ & -1.059356 & -3.422196 & -2.542685 \\
$\mathrm{H}$ & -2.664918 & -4.648284 & -1.221026 \\
$\mathrm{H}$ & -5.460147 & -0.344205 & 2.143975 \\
$\mathrm{H}$ & -5.581561 & -2.075484 & 1.666572 \\
$\mathrm{H}$ & -3.972125 & -1.367406 & 2.093305 \\
$\mathrm{H}$ & -6.834692 & -0.507946 & -0.073910 \\
$\mathrm{H}$ & -5.798033 & 0.939640 & -0.384789 \\
$\mathrm{H}$ & -5.872743 & -0.431113 & -1.573324 \\
$\mathrm{H}$ & 1.811085 & -1.630393 & -3.122931 \\
$\mathrm{H}$ & 1.235925 & -4.061126 & -3.185614 \\
$\mathrm{H}$ & 1.923075 & -5.526685 & -1.297062 \\
\hline \hline & & & \\
\hline
\end{tabular}

Table S15: Cartesian coordinates $(\AA)$ of t2aTS $(\mathrm{E}=$ 1288.718340913698 a.u.).

\begin{tabular}{lrrr}
\hline \hline $\mathrm{C}$ & -0.578468 & -3.071871 & 2.603318 \\
$\mathrm{C}$ & -0.352515 & -2.278635 & 1.473302 \\
$\mathrm{C}$ & 0.790540 & -1.478189 & 1.345141 \\
$\mathrm{C}$ & 1.739207 & -1.498022 & 2.386936 \\
$\mathrm{C}$ & 1.500473 & -2.288923 & 3.521212 \\
$\mathrm{C}$ & 0.351121 & -3.071781 & 3.641471 \\
$\mathrm{Pd}$ & 0.997290 & -0.244156 & -0.272515 \\
$\mathrm{C}$ & 3.037664 & -0.744223 & 2.252029 \\
$\mathrm{C}$ & 4.078636 & -1.548622 & 1.437161
\end{tabular}




\begin{tabular}{|c|c|c|c|}
\hline $\mathrm{C}$ & 3.566207 & -2.158188 & 0.149581 \\
\hline $\mathrm{C}$ & 2.851903 & -1.367770 & -0.796756 \\
\hline $\mathrm{C}$ & 2.540614 & -1.958021 & -2.044513 \\
\hline $\mathrm{C}$ & 2.863361 & -3.284603 & -2.338633 \\
\hline $\mathrm{C}$ & 3.528760 & -4.050927 & -1.381865 \\
\hline $\mathrm{C}$ & 3.888578 & -3.483768 & -0.155671 \\
\hline $\mathrm{H}$ & -1.105929 & -2.263415 & 0.697712 \\
\hline $\mathrm{H}$ & 4.435273 & -4.082198 & 0.571894 \\
\hline $\mathrm{H}$ & 2.237467 & -2.286132 & 4.323963 \\
\hline $\mathrm{H}$ & 0.185782 & -3.670487 & 4.535052 \\
\hline $\mathrm{H}$ & -1.484734 & -3.671854 & 2.669071 \\
\hline $\mathrm{H}$ & 3.398607 & -0.152133 & -0.870388 \\
\hline $\mathrm{H}$ & 3.467463 & -0.527652 & 3.238498 \\
\hline $\mathrm{H}$ & 2.883558 & 0.219202 & 1.761550 \\
\hline $\mathrm{H}$ & 4.496196 & -2.347336 & 2.065274 \\
\hline $\mathrm{H}$ & 4.888266 & -0.846323 & 1.195126 \\
\hline $\mathrm{Br}$ & 1.040824 & 1.440538 & -2.408027 \\
\hline $\mathrm{O}$ & 4.210801 & 0.807702 & -1.182718 \\
\hline $\mathrm{C}$ & 4.497446 & 1.715100 & -0.301336 \\
\hline $\mathrm{O}$ & 4.827734 & 1.583585 & 0.873626 \\
\hline $\mathrm{O}$ & 4.415909 & 2.993295 & -0.821105 \\
\hline $\mathrm{H}$ & 3.981699 & 2.867126 & -1.683402 \\
\hline $\mathrm{P}$ & -0.866559 & 1.096076 & 0.307036 \\
\hline $\mathrm{C}$ & -2.051519 & 1.417811 & -1.072842 \\
\hline $\mathrm{C}$ & -0.200415 & 2.707386 & 0.852426 \\
\hline $\mathrm{C}$ & -1.863932 & 0.686398 & 1.790140 \\
\hline $\mathrm{C}$ & -2.669713 & 0.357020 & -1.801149 \\
\hline $\mathrm{C}$ & -2.322634 & 2.748367 & -1.465623 \\
\hline $\mathrm{C}$ & -1.033941 & 3.679190 & 1.442344 \\
\hline $\mathrm{C}$ & 1.128674 & 2.929908 & 0.784326 \\
\hline $\mathrm{C}$ & -1.232175 & 0.478956 & 2.965331 \\
\hline $\mathrm{C}$ & -3.270174 & 0.733459 & 1.755420 \\
\hline $\mathrm{C}$ & -3.496467 & 0.679437 & -2.890404 \\
\hline $\mathrm{C}$ & -2.343434 & -1.080194 & -1.611635 \\
\hline $\mathrm{C}$ & -3.153847 & 3.028553 & -2.479936 \\
\hline $\mathrm{H}$ & -1.850689 & 3.583125 & -0.978270 \\
\hline $\mathrm{H}$ & -2.099032 & 3.512456 & 1.539385 \\
\hline $\mathrm{C}$ & -0.476512 & 4.878550 & 1.900826 \\
\hline $\mathrm{C}$ & 1.663339 & 4.083948 & 1.230249 \\
\hline $\mathrm{H}$ & 1.783132 & 2.171617 & 0.378033 \\
\hline $\mathrm{H}$ & -0.152486 & 0.512877 & 3.017344 \\
\hline $\mathrm{C}$ & -1.951288 & 0.245349 & 4.143699 \\
\hline $\mathrm{C}$ & -4.009723 & 0.534521 & 2.926437 \\
\hline $\mathrm{H}$ & -3.799968 & 0.949630 & 0.839236 \\
\hline $\mathrm{C}$ & -3.757887 & 2.008351 & -3.209677 \\
\hline $\mathrm{H}$ & -3.910665 & -0.098216 & -3.518898 \\
\hline $\mathrm{C}$ & -3.254675 & -2.000424 & -1.018483 \\
\hline $\mathrm{C}$ & -1.210836 & -1.554273 & -2.177691 \\
\hline $\mathrm{H}$ & -3.339581 & 4.059658 & -2.753716 \\
\hline $\mathrm{C}$ & 0.847130 & 5.078107 & 1.793108 \\
\hline $\mathrm{H}$ & -1.108838 & 5.637282 & 2.343813 \\
\hline
\end{tabular}




\begin{tabular}{rrrr}
$\mathrm{H}$ & 2.731890 & 4.243681 & 1.163810 \\
$\mathrm{C}$ & -3.346684 & 0.271537 & 4.125118 \\
$\mathrm{H}$ & -1.425117 & 0.066826 & 5.072483 \\
$\mathrm{H}$ & -5.090432 & 0.584740 & 2.903817 \\
$\mathrm{H}$ & -4.402142 & 2.248307 & -4.045691 \\
$\mathrm{C}$ & -2.932079 & -3.370533 & -1.024274 \\
$\mathrm{~N}$ & -4.483922 & -1.574076 & -0.399656 \\
$\mathrm{C}$ & -0.907279 & -2.922899 & -2.170828 \\
$\mathrm{H}$ & -0.535673 & -0.885327 & -2.689486 \\
$\mathrm{H}$ & 1.287279 & 6.000970 & 2.148723 \\
$\mathrm{H}$ & -3.908374 & 0.107180 & 5.035420 \\
$\mathrm{C}$ & -1.742872 & -3.806698 & -1.610277 \\
$\mathrm{H}$ & -3.602693 & -4.098155 & -0.586334 \\
$\mathrm{C}$ & -4.797637 & -2.054709 & 0.947346 \\
$\mathrm{C}$ & -5.601231 & -1.129570 & -1.233843 \\
$\mathrm{H}$ & -0.000336 & -3.272653 & -2.643769 \\
$\mathrm{H}$ & -1.500386 & -4.861709 & -1.617797 \\
$\mathrm{H}$ & -5.521644 & -1.371255 & 1.437224 \\
$\mathrm{H}$ & -5.252284 & -3.066025 & 0.891440 \\
$\mathrm{H}$ & -3.875802 & -2.105434 & 1.565877 \\
$\mathrm{H}$ & -6.527560 & -1.683645 & -0.969637 \\
$\mathrm{H}$ & -5.773163 & -0.044607 & -1.075476 \\
$\mathrm{H}$ & -5.402684 & -1.326938 & -2.306576 \\
$\mathrm{H}$ & 2.055970 & -1.341988 & -2.797589 \\
$\mathrm{H}$ & 2.603667 & -3.714726 & -3.304076 \\
$\mathrm{H}$ & 3.785761 & -5.087969 & -1.592291 \\
\hline \hline & & &
\end{tabular}

Table S16: Cartesian coordinates $(\AA)$ of t2bR (E= 1586.418018087886 a.u.).

\begin{tabular}{lrrr}
\hline \hline $\mathrm{C}$ & -0.679761 & -2.014042 & 3.475467 \\
$\mathrm{C}$ & -0.522681 & -1.587718 & 2.152419 \\
$\mathrm{C}$ & 0.566098 & -0.799046 & 1.761927 \\
$\mathrm{C}$ & 1.512037 & -0.406706 & 2.730566 \\
$\mathrm{C}$ & 1.335380 & -0.833548 & 4.056798 \\
$\mathrm{C}$ & 0.253722 & -1.629310 & 4.436058 \\
$\mathrm{Pd}$ & 0.767020 & -0.156075 & -0.172680 \\
$\mathrm{C}$ & 2.734778 & 0.386385 & 2.348641 \\
$\mathrm{C}$ & 3.865819 & -0.532680 & 1.811203 \\
$\mathrm{C}$ & 3.395860 & -1.535483 & 0.790896 \\
$\mathrm{C}$ & 3.111464 & -1.111779 & -0.535174 \\
$\mathrm{C}$ & 2.826230 & -2.092117 & -1.502465 \\
$\mathrm{C}$ & 2.721456 & -3.439090 & -1.166807 \\
$\mathrm{C}$ & 2.935305 & -3.817764 & 0.154698 \\
$\mathrm{C}$ & 3.273552 & -2.888969 & 1.127706 \\
$\mathrm{H}$ & -1.284189 & -1.856444 & 1.433181 \\
$\mathrm{H}$ & 3.455420 & -3.233935 & 2.140135 \\
$\mathrm{H}$ & 2.065483 & -0.523529 & 4.803193 \\
$\mathrm{H}$ & 0.138035 & -1.936344 & 5.473342 \\
$\mathrm{H}$ & -1.536620 & -2.627794 & 3.746523 \\
$\mathrm{H}$ & 3.426595 & -0.103864 & -0.832771
\end{tabular}




\begin{tabular}{|c|c|c|c|}
\hline $\mathrm{H}$ & 3.129459 & 0.942556 & 3.205505 \\
\hline $\mathrm{H}$ & 2.530113 & 1.141318 & 1.585145 \\
\hline $\mathrm{H}$ & 4.320939 & -1.074342 & 2.650526 \\
\hline $\mathrm{H}$ & 4.604183 & 0.125357 & 1.335060 \\
\hline $\mathrm{Br}$ & 0.836080 & 0.807850 & -2.655366 \\
\hline $\mathrm{O}$ & 4.856147 & 1.356440 & -0.474571 \\
\hline $\mathrm{C}$ & 4.662561 & 2.498025 & 0.042396 \\
\hline $\mathrm{O}$ & 4.162033 & 2.819351 & 1.131557 \\
\hline $\mathrm{O}$ & 5.105620 & 3.573209 & -0.762150 \\
\hline $\mathrm{H}$ & 5.424285 & 3.117911 & -1.558527 \\
\hline $\mathrm{F}$ & 2.725823 & -1.783274 & -2.795735 \\
\hline $\mathrm{F}$ & 2.457798 & -4.365752 & -2.107317 \\
\hline $\mathrm{F}$ & 2.827729 & -5.124320 & 0.466224 \\
\hline $\mathrm{P}$ & -1.045131 & 1.256125 & -0.012364 \\
\hline $\mathrm{C}$ & -2.234734 & 1.092868 & -1.415259 \\
\hline $\mathrm{C}$ & -0.417294 & 2.971705 & -0.003112 \\
\hline $\mathrm{C}$ & -2.028323 & 1.289471 & 1.534446 \\
\hline $\mathrm{C}$ & -2.810853 & -0.160653 & -1.786129 \\
\hline $\mathrm{C}$ & -2.542382 & 2.229365 & -2.197908 \\
\hline $\mathrm{C}$ & -1.279381 & 4.052006 & 0.275794 \\
\hline $\mathrm{C}$ & 0.901491 & 3.206449 & -0.172961 \\
\hline $\mathrm{C}$ & -1.398562 & 1.530824 & 2.704696 \\
\hline $\mathrm{C}$ & -3.433268 & 1.217398 & 1.504087 \\
\hline $\mathrm{C}$ & -3.621843 & -0.215740 & -2.932282 \\
\hline $\mathrm{C}$ & -2.468790 & -1.458236 & -1.146823 \\
\hline $\mathrm{C}$ & -3.361733 & 2.156716 & -3.256672 \\
\hline $\mathrm{H}$ & -2.107690 & 3.189922 & -1.984928 \\
\hline $\mathrm{H}$ & -2.335953 & 3.889334 & 0.446165 \\
\hline $\mathrm{C}$ & -0.764234 & 5.352306 & 0.322771 \\
\hline $\mathrm{C}$ & 1.394073 & 4.460659 & -0.127515 \\
\hline $\mathrm{H}$ & 1.583150 & 2.384226 & -0.342353 \\
\hline $\mathrm{H}$ & -0.325656 & 1.663792 & 2.729072 \\
\hline $\mathrm{C}$ & -2.113026 & 1.645592 & 3.903129 \\
\hline $\mathrm{C}$ & -4.170839 & 1.359846 & 2.684866 \\
\hline $\mathrm{H}$ & -3.965605 & 1.081382 & 0.574249 \\
\hline $\mathrm{C}$ & -3.916393 & 0.941272 & -3.646655 \\
\hline $\mathrm{H}$ & -3.998138 & -1.161740 & -3.298122 \\
\hline $\mathrm{C}$ & -3.403212 & -2.187372 & -0.356292 \\
\hline $\mathrm{C}$ & -1.294038 & -2.042690 & -1.473336 \\
\hline $\mathrm{H}$ & -3.574654 & 3.047854 & -3.833936 \\
\hline $\mathrm{C}$ & 0.547623 & 5.552621 & 0.120448 \\
\hline $\mathrm{H}$ & -1.419941 & 6.189782 & 0.523881 \\
\hline $\mathrm{H}$ & 2.452202 & 4.630461 & -0.276306 \\
\hline $\mathrm{C}$ & -3.505758 & 1.557938 & 3.894684 \\
\hline $\mathrm{H}$ & -1.587078 & 1.824947 & 4.831873 \\
\hline $\mathrm{H}$ & -5.251824 & 1.321510 & 2.661219 \\
\hline $\mathrm{H}$ & -4.549394 & 0.890914 & -4.523207 \\
\hline C & -3.060338 & -3.480512 & 0.078595 \\
\hline $\mathrm{N}$ & -4.681689 & -1.642260 & 0.021214 \\
\hline $\mathrm{C}$ & -0.971678 & -3.332974 & -1.029919 \\
\hline $\mathrm{H}$ & -0.595378 & -1.540191 & -2.124057 \\
\hline
\end{tabular}




\begin{tabular}{lrrr}
$\mathrm{H}$ & 0.955276 & 6.554841 & 0.156456 \\
$\mathrm{H}$ & -4.065359 & 1.662135 & 4.815103 \\
$\mathrm{C}$ & -1.828782 & -4.032960 & -0.275437 \\
$\mathrm{H}$ & -3.749012 & -4.060989 & 0.678772 \\
$\mathrm{C}$ & -5.057694 & -1.649002 & 1.435713 \\
$\mathrm{C}$ & -5.765256 & -1.588115 & -0.961178 \\
$\mathrm{H}$ & -0.030729 & -3.777461 & -1.317671 \\
$\mathrm{H}$ & -1.569857 & -5.030784 & 0.055047 \\
$\mathrm{H}$ & -5.784947 & -0.835706 & 1.637883 \\
$\mathrm{H}$ & -5.529782 & -2.619791 & 1.695850 \\
$\mathrm{H}$ & -4.163104 & -1.502330 & 2.078272 \\
$\mathrm{H}$ & -6.678508 & -2.078157 & -0.560254 \\
$\mathrm{H}$ & -5.999865 & -0.529130 & -1.196177 \\
$\mathrm{H}$ & -5.490931 & -2.128573 & -1.889836 \\
\hline \hline
\end{tabular}

Table S17: Cartesian coordinates $(\AA)$ of t2bTS $(\mathrm{E}=$ 1586.409314753652 a.u.).

\begin{tabular}{lrrr}
\hline $\mathrm{C}$ & 0.00372 & -2.45378 & 3.18646 \\
$\mathrm{C}$ & 0.06932 & -1.85338 & 1.92423 \\
$\mathrm{C}$ & 0.84374 & -0.70997 & 1.69303 \\
$\mathrm{C}$ & 1.58039 & -0.16624 & 2.76438 \\
$\mathrm{C}$ & 1.50015 & -0.76921 & 4.02847 \\
$\mathrm{C}$ & 0.71703 & -1.90347 & 4.2497 \\
$\mathrm{Pd}$ & 0.82154 & 0.22114 & -0.12638 \\
$\mathrm{C}$ & 2.51231 & 0.99791 & 2.53417 \\
$\mathrm{C}$ & 3.88081 & 0.52772 & 1.9796 \\
$\mathrm{C}$ & 3.7959 & -0.53115 & 0.90342 \\
$\mathrm{C}$ & 3.05186 & -0.28665 & -0.29301 \\
$\mathrm{C}$ & 3.15107 & -1.2642 & -1.29499 \\
$\mathrm{C}$ & 3.86347 & -2.45176 & -1.13822 \\
$\mathrm{C}$ & 4.533 & -2.66593 & 0.05774 \\
$\mathrm{C}$ & 4.51601 & -1.71441 & 1.07196 \\
$\mathrm{H}$ & -0.52182 & -2.27396 & 1.12195 \\
$\mathrm{H}$ & 5.07842 & -1.91318 & 1.97936 \\
$\mathrm{H}$ & 2.06581 & -0.33494 & 4.85227 \\
$\mathrm{H}$ & 0.66483 & -2.34828 & 5.24124 \\
$\mathrm{H}$ & -0.61498 & -3.33756 & 3.33172 \\
$\mathrm{H}$ & 3.18627 & 0.93867 & -0.68141 \\
$\mathrm{H}$ & 2.6892 & 1.55017 & 3.4655 \\
$\mathrm{H}$ & 2.08175 & 1.70966 & 1.82617 \\
$\mathrm{H}$ & 4.49084 & 0.13808 & 2.80471 \\
$\mathrm{H}$ & 4.37436 & 1.41941 & 1.56918 \\
$\mathrm{Br}$ & 0.49196 & 1.53974 & -2.48045 \\
$\mathrm{O}$ & 3.67462 & 2.07798 & -1.16253 \\
$\mathrm{C}$ & 3.46626 & 3.17208 & -0.49995 \\
$\mathrm{O}$ & 3.77135 & 3.44159 & 0.66158 \\
$\mathrm{O}$ & 2.82151 & 4.13775 & -1.24463 \\
$\mathrm{H}$ & 2.48475 & 3.65163 & -2.02038 \\
$\mathrm{~F}$ & 2.57443 & -1.08893 & -2.4934 \\
$\mathrm{~F}$ & 3.91703 & -3.37279 & -2.12235
\end{tabular}




\begin{tabular}{|c|c|c|c|}
\hline $\mathrm{F}$ & 5.22362 & -3.81385 & 0.21388 \\
\hline $\mathrm{P}$ & -1.41408 & 0.93072 & 0.13097 \\
\hline $\mathrm{C}$ & -2.49616 & 0.60724 & -1.32959 \\
\hline $\mathrm{C}$ & -1.38047 & 2.73564 & 0.41331 \\
\hline $\mathrm{C}$ & -2.36372 & 0.43338 & 1.62012 \\
\hline $\mathrm{C}$ & -2.65966 & -0.70172 & -1.87537 \\
\hline C & -3.14806 & 1.68689 & -1.96807 \\
\hline $\mathrm{C}$ & -2.548 & 3.43683 & 0.77753 \\
\hline $\mathrm{C}$ & -0.20151 & 3.39051 & 0.37698 \\
\hline $\mathrm{C}$ & -1.83618 & 0.66976 & 2.84054 \\
\hline $\mathrm{C}$ & -3.68524 & -0.03975 & 1.5153 \\
\hline $\mathrm{C}$ & -3.43596 & -0.85525 & -3.03641 \\
\hline $\mathrm{C}$ & -1.89822 & -1.89311 & -1.41788 \\
\hline $\mathrm{C}$ & -3.92276 & 1.50412 & -3.04718 \\
\hline $\mathrm{H}$ & -3.02354 & 2.69841 & -1.62376 \\
\hline $\mathrm{H}$ & -3.50141 & 2.92909 & 0.84788 \\
\hline $\mathrm{C}$ & -2.47322 & 4.80889 & 1.04455 \\
\hline $\mathrm{C}$ & -0.13131 & 4.71077 & 0.63835 \\
\hline $\mathrm{H}$ & 0.70696 & 2.85354 & 0.14619 \\
\hline $\mathrm{H}$ & -0.8529 & 1.10912 & 2.93323 \\
\hline $\mathrm{C}$ & -2.55446 & 0.38864 & 4.00887 \\
\hline $\mathrm{C}$ & -4.43445 & -0.29688 & 2.66884 \\
\hline $\mathrm{H}$ & -4.15329 & -0.18342 & 0.55273 \\
\hline $\mathrm{C}$ & -4.08587 & 0.23765 & -3.6016 \\
\hline $\mathrm{H}$ & -3.50759 & -1.81542 & -3.53032 \\
\hline $\mathrm{C}$ & -2.51206 & -2.97458 & -0.72245 \\
\hline $\mathrm{C}$ & -0.61915 & -2.02597 & -1.8349 \\
\hline $\mathrm{H}$ & -4.40774 & 2.35396 & -3.51107 \\
\hline $\mathrm{C}$ & -1.2871 & 5.43442 & 0.97243 \\
\hline $\mathrm{H}$ & -3.36566 & 5.35978 & 1.31253 \\
\hline $\mathrm{H}$ & 0.82474 & 5.21719 & 0.6003 \\
\hline $\mathrm{C}$ & -3.85926 & -0.09898 & 3.92389 \\
\hline $\mathrm{H}$ & -2.10673 & 0.5724 & 4.97694 \\
\hline $\mathrm{H}$ & -5.45586 & -0.64507 & 2.58937 \\
\hline $\mathrm{H}$ & -4.68818 & 0.10927 & -4.49172 \\
\hline $\mathrm{C}$ & -1.75777 & -4.13755 & -0.47901 \\
\hline $\mathrm{N}$ & -3.87119 & -2.9122 & -0.24849 \\
\hline $\mathrm{C}$ & 0.11448 & -3.19398 & -1.5821 \\
\hline $\mathrm{H}$ & -0.15208 & -1.24472 & -2.41631 \\
\hline $\mathrm{H}$ & -1.21922 & 6.49445 & 1.18122 \\
\hline $\mathrm{H}$ & -4.42615 & -0.30254 & 4.82297 \\
\hline $\mathrm{C}$ & -0.43769 & -4.22078 & -0.92339 \\
\hline $\mathrm{H}$ & -2.19361 & -4.98008 & 0.04174 \\
\hline $\mathrm{C}$ & -4.14633 & -3.26708 & 1.1452 \\
\hline $\mathrm{C}$ & -4.97771 & -3.01717 & -1.20064 \\
\hline $\mathrm{H}$ & 1.12938 & -3.27865 & -1.94328 \\
\hline $\mathrm{H}$ & 0.13742 & -5.11959 & -0.74082 \\
\hline $\mathrm{H}$ & -5.10278 & -2.81021 & 1.47328 \\
\hline $\mathrm{H}$ & -4.23175 & -4.36954 & 1.24475 \\
\hline $\mathrm{H}$ & -3.32934 & -2.90619 & 1.80643 \\
\hline $\mathrm{H}$ & -5.69117 & -3.80737 & -0.88203 \\
\hline
\end{tabular}




\begin{tabular}{lrrr}
$\mathrm{H}$ & -5.515 & -2.04745 & -1.25271 \\
$\mathrm{H}$ & -4.61261 & -3.30087 & -2.20828 \\
\hline
\end{tabular}

Table S18: Cartesian coordinates $(\AA)$ of t2cR $(\mathrm{E}=$ 1387.962117717459 a.u.).

\begin{tabular}{lrrr}
\hline \hline $\mathrm{C}$ & -0.940523 & -2.514954 & 3.117716 \\
$\mathrm{C}$ & -0.716967 & -1.952916 & 1.856270 \\
$\mathrm{C}$ & 0.491875 & -1.323322 & 1.539577 \\
$\mathrm{C}$ & 1.500911 & -1.237995 & 2.520067 \\
$\mathrm{C}$ & 1.258011 & -1.797254 & 3.785208 \\
$\mathrm{C}$ & 0.052633 & -2.430407 & 4.091822 \\
$\mathrm{Pd}$ & 0.779300 & -0.473419 & -0.300230 \\
$\mathrm{C}$ & 2.846364 & -0.637635 & 2.203117 \\
$\mathrm{C}$ & 3.777959 & -1.668664 & 1.506100 \\
$\mathrm{C}$ & 3.105917 & -2.427162 & 0.392786 \\
$\mathrm{C}$ & 2.883452 & -1.793799 & -0.859960 \\
$\mathrm{C}$ & 2.376052 & -2.579591 & -1.911684 \\
$\mathrm{C}$ & 1.998167 & -3.908286 & -1.745149 \\
$\mathrm{C}$ & 2.167389 & -4.491334 & -0.489344 \\
$\mathrm{C}$ & 2.719815 & -3.763461 & 0.563827 \\
$\mathrm{H}$ & -1.513716 & -1.990446 & 1.126429 \\
$\mathrm{H}$ & 2.870119 & -4.237675 & 1.529849 \\
$\mathrm{H}$ & 2.037454 & -1.724820 & 4.542477 \\
$\mathrm{H}$ & -0.110067 & -2.844872 & 5.084616 \\
$\mathrm{H}$ & -1.891855 & -2.998082 & 3.332934 \\
$\mathrm{H}$ & 3.374266 & -0.833361 & -1.051079 \\
$\mathrm{H}$ & 3.347472 & -0.285682 & 3.110950 \\
$\mathrm{H}$ & 2.776226 & 0.239143 & 1.554165 \\
$\mathrm{H}$ & 4.138235 & -2.388783 & 2.252622 \\
$\mathrm{H}$ & 4.617156 & -1.098050 & 1.087628 \\
$\mathrm{Br}$ & 0.980511 & 0.796016 & -2.638874 \\
$\mathrm{O}$ & 5.092114 & 0.312146 & -0.516060 \\
$\mathrm{C}$ & 5.138342 & 1.382368 & 0.160345 \\
$\mathrm{O}$ & 4.730688 & 1.638536 & 1.304342 \\
$\mathrm{O}$ & 5.774044 & 2.455976 & -0.510740 \\
$\mathrm{H}$ & 5.979595 & 2.063129 & -1.374768 \\
$\mathrm{~F}$ & 2.320071 & -2.074083 & -3.151543 \\
$\mathrm{P}$ & -0.720383 & 1.224803 & 0.127577 \\
$\mathrm{C}$ & -1.946571 & 1.492051 & -1.226902 \\
$\mathrm{C}$ & 0.229125 & 2.772082 & 0.337069 \\
$\mathrm{C}$ & -1.651369 & 1.232561 & 1.707090 \\
$\mathrm{C}$ & -2.764823 & 0.436369 & -1.733805 \\
$\mathrm{C}$ & -2.049967 & 2.767562 & -1.827852 \\
$\mathrm{C}$ & -0.399658 & 3.946024 & 0.800703 \\
$\mathrm{C}$ & -0.964152 & 2.774511 & 0.134482 \\
$\mathrm{C}$ & -3.043442 & 1.185068 & 2.870323 \\
$\mathrm{C}$ & -2.6521596951 & -0.984339 & -1.311215 \\
$\mathrm{C}$ & 3.005355 & -2.837585
\end{tabular}




\begin{tabular}{rrrr}
$\mathrm{H}$ & -1.431093 & 3.587909 & -1.510189 \\
$\mathrm{H}$ & -1.463510 & 3.964522 & 0.999985 \\
$\mathrm{C}$ & 0.359450 & 5.105429 & 0.995245 \\
$\mathrm{C}$ & 2.291973 & 3.893766 & 0.322281 \\
$\mathrm{H}$ & 2.069124 & 1.870421 & -0.177324 \\
$\mathrm{H}$ & 0.111652 & 1.107022 & 2.865177 \\
$\mathrm{C}$ & -1.624008 & 1.268696 & 4.103562 \\
$\mathrm{C}$ & -3.718218 & 1.555949 & 2.949529 \\
$\mathrm{H}$ & -3.608472 & 1.537366 & 0.814335 \\
$\mathrm{C}$ & -3.692873 & 1.986450 & -3.356726 \\
$\mathrm{H}$ & -4.172175 & -0.091957 & -3.295433 \\
$\mathrm{C}$ & -3.666408 & -1.639294 & -0.554267 \\
$\mathrm{C}$ & -1.640063 & -1.721672 & -1.821412 \\
$\mathrm{H}$ & -2.952487 & 3.995047 & -3.273955 \\
$\mathrm{C}$ & 1.680043 & 5.080254 & 0.755977 \\
$\mathrm{H}$ & -0.116054 & 6.015105 & 1.338921 \\
$\mathrm{H}$ & 3.359037 & 3.879646 & 0.144335 \\
$\mathrm{C}$ & -3.006723 & 1.452566 & 4.144634 \\
$\mathrm{H}$ & -1.057134 & 1.214219 & 5.023886 \\
$\mathrm{H}$ & -4.785970 & 1.729973 & 2.967949 \\
$\mathrm{H}$ & -4.350771 & 2.184577 & -4.193093 \\
$\mathrm{C}$ & -3.565862 & -3.027626 & -0.345790 \\
$\mathrm{~N}$ & -4.780073 & -0.926917 & 0.018044 \\
$\mathrm{C}$ & -1.557342 & -3.103136 & -1.599786 \\
$\mathrm{H}$ & -0.896464 & -1.264709 & -2.455667 \\
$\mathrm{H}$ & 2.275368 & 5.972021 & 0.904891 \\
$\mathrm{H}$ & -3.519093 & 1.534139 & 5.094361 \\
$\mathrm{C}$ & -2.492258 & -3.739317 & -0.882609 \\
$\mathrm{H}$ & -4.320354 & -3.557215 & 0.221131 \\
$\mathrm{C}$ & -5.083138 & -1.120397 & 1.437388 \\
$\mathrm{C}$ & -5.868068 & -0.466075 & -0.845522 \\
$\mathrm{H}$ & -0.744777 & -3.666345 & -2.034871 \\
$\mathrm{H}$ & -2.420776 & -4.808009 & -0.725594 \\
$\mathrm{H}$ & -5.658175 & -0.254440 & 1.825441 \\
$\mathrm{H}$ & -5.694710 & -2.037283 & 1.571657 \\
$\mathrm{H}$ & -4.145329 & -1.222452 & 2.024751 \\
$\mathrm{H}$ & -6.848290 & -0.815736 & -0.455875 \\
$\mathrm{H}$ & -5.869550 & 0.643238 & -0.879393 \\
$\mathrm{H}$ & -5.765089 & -0.874522 & -1.871081 \\
\hline & 1.617165 & -4.464339 & -2.595700 \\
\hline & & & \\
\hline
\end{tabular}

Table S19: Cartesian coordinates $(\AA)$ of t2cTS $(\mathrm{E}=$ 1387.951218547671 a.u.).

\begin{tabular}{lrrr}
\hline \hline $\mathrm{C}$ & -0.513293 & -2.946510 & 2.771838 \\
$\mathrm{C}$ & -0.305107 & -2.219613 & 1.594362 \\
$\mathrm{C}$ & 0.772802 & -1.336751 & 1.455306 \\
$\mathrm{C}$ & 1.671043 & -1.195281 & 2.532046 \\
$\mathrm{C}$ & 1.448916 & -1.920778 & 3.711809 \\
$\mathrm{C}$ & 0.365055 & -2.790918 & 3.842610
\end{tabular}




\begin{tabular}{|c|c|c|c|}
\hline $\mathrm{Pd}$ & 0.977789 & -0.189738 & -0.223029 \\
\hline $\mathrm{C}$ & 2.903806 & -0.338266 & 2.384868 \\
\hline $\mathrm{C}$ & 4.046545 & -1.107425 & 1.675101 \\
\hline $\mathrm{C}$ & 3.613673 & -1.932793 & 0.483702 \\
\hline $\mathrm{C}$ & 2.920251 & -1.318592 & -0.604758 \\
\hline $\mathrm{C}$ & 2.702332 & -2.139240 & -1.723647 \\
\hline $\mathrm{C}$ & 3.046454 & -3.487250 & -1.795784 \\
\hline $\mathrm{C}$ & 3.676104 & -4.059449 & -0.694467 \\
\hline $\mathrm{C}$ & 3.969980 & -3.282745 & 0.430110 \\
\hline $\mathrm{H}$ & -1.019505 & -2.326092 & 0.789155 \\
\hline $\mathrm{H}$ & 4.489300 & -3.732957 & 1.273209 \\
\hline $\mathrm{H}$ & 2.144147 & -1.796376 & 4.541551 \\
\hline $\mathrm{H}$ & 0.209498 & -3.336827 & 4.770957 \\
\hline $\mathrm{H}$ & -1.367445 & -3.617307 & 2.847887 \\
\hline $\mathrm{H}$ & 3.408437 & -0.135857 & -0.845593 \\
\hline $\mathrm{H}$ & 3.267945 & -0.001311 & 3.363555 \\
\hline $\mathrm{H}$ & 2.683081 & 0.561128 & 1.805744 \\
\hline $\mathrm{H}$ & 4.539082 & -1.770339 & 2.398559 \\
\hline $\mathrm{H}$ & 4.773418 & -0.352488 & 1.344267 \\
\hline $\mathrm{Br}$ & 0.978944 & 1.503492 & -2.364484 \\
\hline $\mathrm{O}$ & 4.207027 & 0.850807 & -1.210900 \\
\hline $\mathrm{C}$ & 4.362581 & 1.861469 & -0.412550 \\
\hline $\mathrm{O}$ & 4.815465 & 1.876880 & 0.731410 \\
\hline $\mathrm{O}$ & 3.964573 & 3.056981 & -0.972788 \\
\hline $\mathrm{H}$ & 3.437914 & 2.791394 & -1.750950 \\
\hline $\mathrm{F}$ & 2.145699 & -1.611941 & -2.833074 \\
\hline $\mathrm{P}$ & -0.939989 & 1.102383 & 0.283229 \\
\hline $\mathrm{C}$ & -2.114491 & 1.324318 & -1.124193 \\
\hline $\mathrm{C}$ & -0.359340 & 2.758270 & 0.792704 \\
\hline $\mathrm{C}$ & -1.949384 & 0.697121 & 1.761356 \\
\hline $\mathrm{C}$ & -2.681971 & 0.213464 & -1.818360 \\
\hline $\mathrm{C}$ & -2.430208 & 2.627640 & -1.571348 \\
\hline $\mathrm{C}$ & -1.251987 & 3.710908 & 1.324828 \\
\hline $\mathrm{C}$ & 0.960282 & 3.036195 & 0.760116 \\
\hline $\mathrm{C}$ & -1.337456 & 0.587611 & 2.960149 \\
\hline $\mathrm{C}$ & -3.354727 & 0.655949 & 1.693418 \\
\hline $\mathrm{C}$ & -3.505065 & 0.462719 & -2.929485 \\
\hline $\mathrm{C}$ & -2.300238 & -1.201714 & -1.573011 \\
\hline $\mathrm{C}$ & -3.257979 & 2.837094 & -2.605246 \\
\hline $\mathrm{H}$ & -1.995182 & 3.497654 & -1.111926 \\
\hline $\mathrm{H}$ & -2.311502 & 3.500585 & 1.395062 \\
\hline $\mathrm{C}$ & -0.761310 & 4.947057 & 1.761457 \\
\hline $\mathrm{C}$ & 1.430958 & 4.225512 & 1.184784 \\
\hline $\mathrm{H}$ & 1.658472 & 2.294776 & 0.399991 \\
\hline $\mathrm{H}$ & -0.264908 & 0.698472 & 3.036461 \\
\hline $\mathrm{C}$ & -2.070504 & 0.364912 & 4.131906 \\
\hline C & -4.110445 & 0.467359 & 2.855929 \\
\hline $\mathrm{H}$ & -3.873982 & 0.798040 & 0.757226 \\
\hline $\mathrm{C}$ & -3.813071 & 1.767508 & -3.302258 \\
\hline $\mathrm{H}$ & -3.878427 & -0.352951 & -3.534697 \\
\hline $\mathrm{C}$ & -3.188449 & -2.143056 & -0.977240 \\
\hline
\end{tabular}




\begin{tabular}{lrrr}
$\mathrm{C}$ & -1.134351 & -1.643165 & -2.096016 \\
$\mathrm{H}$ & -3.478562 & 3.849176 & -2.920944 \\
$\mathrm{C}$ & 0.555475 & 5.200459 & 1.688948 \\
$\mathrm{H}$ & -1.439380 & 5.690854 & 2.159864 \\
$\mathrm{H}$ & 2.493583 & 4.428798 & 1.146818 \\
$\mathrm{C}$ & -3.463667 & 0.302283 & 4.080506 \\
$\mathrm{H}$ & -1.558306 & 0.265701 & 5.080165 \\
$\mathrm{H}$ & -5.191291 & 0.451805 & 2.807192 \\
$\mathrm{H}$ & -4.454174 & 1.950634 & -4.154909 \\
$\mathrm{C}$ & -2.811779 & -3.498559 & -0.939449 \\
$\mathrm{~N}$ & -4.449587 & -1.752707 & -0.400175 \\
$\mathrm{C}$ & -0.778623 & -2.998977 & -2.048242 \\
$\mathrm{H}$ & -0.472622 & -0.958110 & -2.605542 \\
$\mathrm{H}$ & 0.944301 & 6.152089 & 2.028181 \\
$\mathrm{H}$ & -4.037383 & 0.147111 & 4.984904 \\
$\mathrm{C}$ & -1.593031 & -3.901400 & -1.486541 \\
$\mathrm{H}$ & -3.464036 & -4.241395 & -0.499300 \\
$\mathrm{C}$ & -4.773931 & -2.207835 & 0.953238 \\
$\mathrm{C}$ & -5.566313 & -1.389209 & -1.273561 \\
$\mathrm{H}$ & 0.151785 & -3.326158 & -2.490481 \\
$\mathrm{H}$ & -1.309768 & -4.945960 & -1.462264 \\
$\mathrm{H}$ & -5.538019 & -1.542938 & 1.406291 \\
$\mathrm{H}$ & -5.183378 & -3.239184 & 0.917277 \\
$\mathrm{H}$ & -3.865554 & -2.201078 & 1.593355 \\
$\mathrm{H}$ & -6.474356 & -1.971474 & -1.006412 \\
$\mathrm{H}$ & -5.787196 & -0.307322 & -1.161801 \\
$\mathrm{H}$ & -5.336623 & -1.619995 & -2.333322 \\
$\mathrm{H}$ & 2.826728 & -4.051820 & -2.696927 \\
$\mathrm{H}$ & 3.954488 & -5.111164 & -0.717237 \\
\hline \hline & & &
\end{tabular}

Table S20: Cartesian coordinates $(\AA)$ of t2dR $(E=-$ 1387.967946116538 a.u.).

\begin{tabular}{lrrr}
\hline \hline $\mathrm{C}$ & -0.773487 & -2.269206 & 3.294808 \\
$\mathrm{C}$ & -0.603295 & -1.784908 & 1.993998 \\
$\mathrm{C}$ & 0.591603 & -1.180343 & 1.586140 \\
$\mathrm{C}$ & 1.641507 & -1.033040 & 2.515555 \\
$\mathrm{C}$ & 1.449638 & -1.516187 & 3.821258 \\
$\mathrm{C}$ & 0.261176 & -2.129361 & 4.217689 \\
$\mathrm{Pd}$ & 0.762007 & -0.477242 & -0.328922 \\
$\mathrm{C}$ & 2.972562 & -0.433163 & 2.135658 \\
$\mathrm{C}$ & 3.932131 & -1.490683 & 1.521179 \\
$\mathrm{C}$ & 3.266995 & -2.319129 & 0.457019 \\
$\mathrm{C}$ & 2.868803 & -1.706271 & -0.759792 \\
$\mathrm{C}$ & 2.236524 & -2.467838 & -1.765035 \\
$\mathrm{C}$ & 2.016013 & -3.823497 & -1.545364 \\
$\mathrm{C}$ & 2.389972 & -4.442105 & -0.361988 \\
$\mathrm{C}$ & 3.007648 & -3.680374 & 0.635286 \\
$\mathrm{H}$ & -1.433051 & -1.858984 & 1.305232 \\
$\mathrm{H}$ & 3.298528 & -4.160690 & 1.566304 \\
$\mathrm{H}$ & 2.259462 & -1.395921 & 4.539505
\end{tabular}




\begin{tabular}{|c|c|c|c|}
\hline $\mathrm{H}$ & 0.142727 & -2.483032 & 5.239766 \\
\hline $\mathrm{H}$ & -1.715606 & -2.734205 & 3.578875 \\
\hline $\mathrm{H}$ & 3.266391 & -0.707466 & -0.974443 \\
\hline $\mathrm{H}$ & 3.465081 & 0.007261 & 3.008881 \\
\hline $\mathrm{H}$ & 2.877863 & 0.386619 & 1.419556 \\
\hline $\mathrm{H}$ & 4.309365 & -2.151267 & 2.313354 \\
\hline $\mathrm{H}$ & 4.758082 & -0.927751 & 1.068319 \\
\hline $\mathrm{Br}$ & 0.850703 & 0.563952 & -2.812071 \\
\hline $\mathrm{O}$ & 5.052193 & 0.473563 & -0.632989 \\
\hline $\mathrm{C}$ & 5.175059 & 1.554471 & 0.015870 \\
\hline $\mathrm{O}$ & 4.758766 & 1.882254 & 1.137987 \\
\hline $\mathrm{O}$ & 5.925571 & 2.546565 & -0.664839 \\
\hline $\mathrm{H}$ & 6.126154 & 2.106615 & -1.507078 \\
\hline $\mathrm{P}$ & -0.719418 & 1.253585 & 0.042836 \\
\hline $\mathrm{C}$ & -2.004765 & 1.420977 & -1.272119 \\
\hline $\mathrm{C}$ & 0.246618 & 2.804040 & 0.073814 \\
\hline $\mathrm{C}$ & -1.572069 & 1.394166 & 1.658690 \\
\hline $\mathrm{C}$ & -2.844218 & 0.332042 & -1.659862 \\
\hline C & -2.129635 & 2.644514 & -1.969449 \\
\hline $\mathrm{C}$ & -0.348319 & 4.015908 & 0.480743 \\
\hline $\mathrm{C}$ & 1.564947 & 2.780352 & -0.216989 \\
\hline $\mathrm{C}$ & -0.832536 & 1.424663 & 2.788285 \\
\hline $\mathrm{C}$ & -2.959754 & 1.616097 & 1.730943 \\
\hline C & -3.722415 & 0.511204 & -2.741963 \\
\hline $\mathrm{C}$ & -2.730049 & -1.046117 & -1.114414 \\
\hline $\mathrm{C}$ & -3.014310 & 2.802696 & -2.964496 \\
\hline $\mathrm{H}$ & -1.500323 & 3.486232 & -1.740664 \\
\hline $\mathrm{H}$ & -1.397070 & 4.058366 & 0.745372 \\
\hline C & 0.423976 & 5.181833 & 0.529952 \\
\hline $\mathrm{C}$ & 2.304958 & 3.906468 & -0.170979 \\
\hline $\mathrm{H}$ & 2.046199 & 1.850235 & -0.487949 \\
\hline $\mathrm{H}$ & 0.243547 & 1.332517 & 2.737940 \\
\hline C & -1.429119 & 1.607853 & 4.041458 \\
\hline C & -3.574156 & 1.832564 & 2.969503 \\
\hline $\mathrm{H}$ & -3.566992 & 1.651968 & 0.838465 \\
\hline $\mathrm{C}$ & -3.824956 & 1.747326 & -3.372251 \\
\hline $\mathrm{H}$ & -4.305232 & -0.316818 & -3.123551 \\
\hline $\mathrm{C}$ & -3.725802 & -1.617291 & -0.270303 \\
\hline $\mathrm{C}$ & -1.737129 & -1.837155 & -1.579958 \\
\hline $\mathrm{H}$ & -3.082629 & 3.754190 & -3.476995 \\
\hline $\mathrm{C}$ & 1.725422 & 5.128298 & 0.205375 \\
\hline $\mathrm{H}$ & -0.026126 & 6.119730 & 0.829121 \\
\hline $\mathrm{H}$ & 3.356925 & 3.871469 & -0.420840 \\
\hline $\mathrm{C}$ & -2.806739 & 1.810897 & 4.133947 \\
\hline $\mathrm{H}$ & -0.819231 & 1.614880 & 4.935504 \\
\hline $\mathrm{H}$ & -4.638390 & 2.019532 & 3.025482 \\
\hline $\mathrm{H}$ & -4.511401 & 1.879151 & -4.198684 \\
\hline C & -3.627100 & -2.979609 & 0.068103 \\
\hline $\mathrm{N}$ & -4.822611 & -0.846061 & 0.256407 \\
\hline $\mathrm{C}$ & -1.655802 & -3.191834 & -1.228579 \\
\hline $\mathrm{H}$ & -1.007405 & -1.448204 & -2.272591 \\
\hline
\end{tabular}




\begin{tabular}{lrrr}
$\mathrm{H}$ & 2.330631 & 6.025156 & 0.240438 \\
$\mathrm{H}$ & -3.272192 & 1.969192 & 5.098028 \\
$\mathrm{C}$ & -2.573952 & -3.748742 & -0.428374 \\
$\mathrm{H}$ & -4.368425 & -3.445579 & 0.704096 \\
$\mathrm{C}$ & -5.088108 & -0.893922 & 1.695262 \\
$\mathrm{C}$ & -5.934053 & -0.472729 & -0.619620 \\
$\mathrm{H}$ & -0.855666 & -3.799728 & -1.624175 \\
$\mathrm{H}$ & -2.503338 & -4.797686 & -0.170253 \\
$\mathrm{H}$ & -5.629884 & 0.021122 & 2.012153 \\
$\mathrm{H}$ & -5.717237 & -1.777201 & 1.933757 \\
$\mathrm{H}$ & -4.136568 & -0.965468 & 2.264682 \\
$\mathrm{H}$ & -6.903009 & -0.780844 & -0.171168 \\
$\mathrm{H}$ & -5.938718 & 0.627687 & -0.763854 \\
$\mathrm{H}$ & -5.857761 & -0.981657 & -1.601727 \\
$\mathrm{H}$ & 2.197930 & -5.503017 & -0.229585 \\
$\mathrm{~F}$ & 1.442695 & -4.570695 & -2.523181 \\
$\mathrm{H}$ & 1.970785 & -2.012960 & -2.712384 \\
\hline \hline
\end{tabular}

Table S21: Cartesian coordinates $(\AA)$ of t2dTS $(\mathrm{E}=$ 1387.953484570161 a.u.).

\begin{tabular}{lrrr}
\hline \hline $\mathrm{C}$ & -0.432740 & -2.524993 & 3.156278 \\
$\mathrm{C}$ & -0.239530 & -1.926237 & 1.906703 \\
$\mathrm{C}$ & 0.811843 & -1.030871 & 1.670346 \\
$\mathrm{C}$ & 1.701744 & -0.747722 & 2.725437 \\
$\mathrm{C}$ & 1.493025 & -1.343948 & 3.978538 \\
$\mathrm{C}$ & 0.434527 & -2.225091 & 4.205005 \\
$\mathrm{Pd}$ & 0.975518 & -0.098733 & -0.142024 \\
$\mathrm{C}$ & 2.917754 & 0.114017 & 2.493767 \\
$\mathrm{C}$ & 4.081427 & -0.701358 & 1.877620 \\
$\mathrm{C}$ & 3.699668 & -1.606822 & 0.726041 \\
$\mathrm{C}$ & 2.967472 & -1.097968 & -0.385073 \\
$\mathrm{C}$ & 2.771849 & -1.942506 & -1.502254 \\
$\mathrm{C}$ & 3.242664 & -3.247940 & -1.484130 \\
$\mathrm{C}$ & 3.924831 & -3.761712 & -0.390282 \\
$\mathrm{C}$ & 4.158084 & -2.926785 & 0.707012 \\
$\mathrm{H}$ & -0.947803 & -2.142717 & 1.118252 \\
$\mathrm{H}$ & 4.716808 & -3.315019 & 1.556803 \\
$\mathrm{H}$ & 2.181508 & -1.108723 & 4.789737 \\
$\mathrm{H}$ & 0.290675 & -2.668532 & 5.188229 \\
$\mathrm{H}$ & -1.267149 & -3.208690 & 3.303514 \\
$\mathrm{H}$ & 3.384453 & 0.120597 & -0.669785 \\
$\mathrm{H}$ & 3.268732 & 0.554116 & 3.435865 \\
$\mathrm{H}$ & 2.688884 & 0.949599 & 1.828335 \\
$\mathrm{H}$ & 4.554842 & -1.308036 & 2.661478 \\
$\mathrm{H}$ & 4.818382 & 0.031756 & 1.521635 \\
$\mathrm{Br}$ & 0.980147 & 1.166730 & -2.541446 \\
$\mathrm{O}$ & 4.150557 & 1.082413 & -1.110741 \\
$\mathrm{C}$ & 4.218188 & 2.189731 & -0.435848 \\
$\mathrm{O}$ & 4.556489 & 2.363025 & 0.731893 \\
$\mathrm{O}$ & 3.861077 & 3.290731 & -1.188602
\end{tabular}




\begin{tabular}{|c|c|c|c|}
\hline $\mathrm{H}$ & 3.445325 & 2.905987 & -1.982074 \\
\hline $\mathrm{P}$ & -1.041856 & 1.109212 & 0.122266 \\
\hline $\mathrm{C}$ & -2.170310 & 1.053198 & -1.338397 \\
\hline $\mathrm{C}$ & -0.579536 & 2.854648 & 0.396872 \\
\hline $\mathrm{C}$ & -2.076433 & 0.856057 & 1.614637 \\
\hline $\mathrm{C}$ & -2.641908 & -0.179527 & -1.881910 \\
\hline $\mathrm{C}$ & -2.541203 & 2.255973 & -1.981817 \\
\hline $\mathrm{C}$ & -1.543646 & 3.815108 & 0.764670 \\
\hline $\mathrm{C}$ & 0.721196 & 3.208195 & 0.349843 \\
\hline $\mathrm{C}$ & -1.496345 & 0.928729 & 2.831861 \\
\hline $\mathrm{C}$ & -3.476184 & 0.745511 & 1.517416 \\
\hline $\mathrm{C}$ & -3.427867 & -0.145981 & -3.046028 \\
\hline $\mathrm{C}$ & -2.192760 & -1.517008 & -1.415679 \\
\hline $\mathrm{C}$ & -3.333197 & 2.261335 & -3.063899 \\
\hline $\mathrm{H}$ & -2.178785 & 3.209094 & -1.638991 \\
\hline $\mathrm{H}$ & -2.590329 & 3.550229 & 0.841562 \\
\hline $\mathrm{C}$ & -1.141336 & 5.130013 & 1.026454 \\
\hline $\mathrm{C}$ & 1.106624 & 4.473959 & 0.606930 \\
\hline $\mathrm{H}$ & 1.472347 & 2.467541 & 0.115577 \\
\hline $\mathrm{H}$ & -0.430686 & 1.090025 & 2.919303 \\
\hline $\mathrm{C}$ & -2.255390 & 0.829611 & 4.004129 \\
\hline $\mathrm{C}$ & -4.258716 & 0.678174 & 2.675438 \\
\hline $\mathrm{H}$ & -3.971306 & 0.740812 & 0.557431 \\
\hline $\mathrm{C}$ & -3.793716 & 1.069380 & -3.616517 \\
\hline $\mathrm{H}$ & -3.727801 & -1.062697 & -3.536854 \\
\hline $\mathrm{C}$ & -3.045380 & -2.405562 & -0.699232 \\
\hline $\mathrm{C}$ & -0.988212 & -1.963660 & -1.836805 \\
\hline $\mathrm{H}$ & -3.598148 & 3.201390 & -3.531590 \\
\hline $\mathrm{C}$ & 0.159497 & 5.453566 & 0.945564 \\
\hline $\mathrm{H}$ & -1.874446 & 5.878840 & 1.297656 \\
\hline $\mathrm{H}$ & 2.155522 & 4.737515 & 0.562685 \\
\hline $\mathrm{C}$ & -3.643096 & 0.702187 & 3.926796 \\
\hline $\mathrm{H}$ & -1.768549 & 0.875476 & 4.969663 \\
\hline $\mathrm{H}$ & -5.336104 & 0.608997 & 2.602147 \\
\hline $\mathrm{H}$ & -4.406178 & 1.086806 & -4.508785 \\
\hline $\mathrm{C}$ & -2.592696 & -3.712356 & -0.436995 \\
\hline $\mathrm{N}$ & -4.346247 & -2.007840 & -0.223750 \\
\hline $\mathrm{C}$ & -0.556539 & -3.269208 & -1.564400 \\
\hline $\mathrm{H}$ & -0.350323 & -1.330302 & -2.434801 \\
\hline $\mathrm{H}$ & 0.480227 & 6.466925 & 1.150770 \\
\hline $\mathrm{H}$ & -4.237327 & 0.641566 & 4.829225 \\
\hline $\mathrm{C}$ & -1.335104 & -4.120037 & -0.883868 \\
\hline $\mathrm{H}$ & -3.215153 & -4.415768 & 0.100281 \\
\hline $\mathrm{C}$ & -4.699190 & -2.278251 & 1.171387 \\
\hline $\mathrm{C}$ & -5.445263 & -1.832551 & -1.174246 \\
\hline $\mathrm{H}$ & 0.405523 & -3.601531 & -1.927159 \\
\hline $\mathrm{H}$ & -0.992804 & -5.127812 & -0.686063 \\
\hline $\mathrm{H}$ & -5.512058 & -1.597244 & 1.498049 \\
\hline $\mathrm{H}$ & -5.055516 & -3.324619 & 1.275096 \\
\hline $\mathrm{H}$ & -3.817446 & -2.128541 & 1.830996 \\
\hline $\mathrm{H}$ & -6.333620 & -2.418142 & -0.853586 \\
\hline
\end{tabular}




\begin{tabular}{lrrr}
$\mathrm{H}$ & -5.722046 & -0.759052 & -1.226929 \\
$\mathrm{H}$ & -5.164680 & -2.199906 & -2.181889 \\
$\mathrm{H}$ & 4.278284 & -4.788848 & -0.411008 \\
$\mathrm{~F}$ & 3.042683 & -4.053751 & -2.563041 \\
$\mathrm{H}$ & 2.275664 & -1.561644 & -2.390041 \\
\hline \hline
\end{tabular}

Table S22: Cartesian coordinates $(\AA)$ of t2eR $(\mathrm{E}=$ 1387.971245769174 a.u.).

\begin{tabular}{lrrr}
\hline \hline $\mathrm{C}$ & -0.623069 & -2.643080 & 2.956225 \\
$\mathrm{C}$ & -0.483872 & -2.008890 & 1.717606 \\
$\mathrm{C}$ & 0.672872 & -1.295432 & 1.383116 \\
$\mathrm{C}$ & 1.719024 & -1.200715 & 2.323872 \\
$\mathrm{C}$ & 1.558731 & -1.834833 & 3.567566 \\
$\mathrm{C}$ & 0.405482 & -2.549739 & 3.891641 \\
$\mathrm{Pd}$ & 0.776329 & -0.362052 & -0.435017 \\
$\mathrm{C}$ & 3.017517 & -0.500013 & 2.007747 \\
$\mathrm{C}$ & 4.009364 & -1.431231 & 1.252074 \\
$\mathrm{C}$ & 3.350604 & -2.162880 & 0.117792 \\
$\mathrm{C}$ & 2.885449 & -1.432558 & -1.011351 \\
$\mathrm{C}$ & 2.267930 & -2.118417 & -2.079835 \\
$\mathrm{C}$ & 2.089148 & -3.504292 & -2.039135 \\
$\mathrm{C}$ & 2.544329 & -4.183130 & -0.918716 \\
$\mathrm{C}$ & 3.163312 & -3.544654 & 0.152771 \\
$\mathrm{H}$ & -1.307454 & -2.054847 & 1.019332 \\
$\mathrm{H}$ & 3.493458 & -4.134813 & 1.002307 \\
$\mathrm{H}$ & 2.364699 & -1.754353 & 4.295570 \\
$\mathrm{H}$ & 0.310234 & -3.020639 & 4.867801 \\
$\mathrm{H}$ & -1.536716 & -3.188860 & 3.183989 \\
$\mathrm{H}$ & 3.253251 & -0.406901 & -1.127970 \\
$\mathrm{H}$ & 3.508699 & -0.155510 & 2.923779 \\
$\mathrm{H}$ & 2.876843 & 0.399435 & 1.403477 \\
$\mathrm{H}$ & 4.441879 & -2.157873 & 1.952310 \\
$\mathrm{H}$ & 4.791207 & -0.778204 & 0.841238 \\
$\mathrm{Br}$ & 0.762613 & 0.962783 & -2.783785 \\
$\mathrm{O}$ & 5.010007 & 0.799865 & -0.665721 \\
$\mathrm{C}$ & 5.072599 & 1.819940 & 0.083016 \\
$\mathrm{O}$ & 4.658996 & 2.007992 & 1.237666 \\
$\mathrm{O}$ & 5.738547 & 2.922561 & -0.508904 \\
$\mathrm{H}$ & 5.951286 & 2.580744 & -1.392661 \\
$\mathrm{P}$ & -0.798688 & 1.232492 & 0.149082 \\
$\mathrm{C}$ & -2.114237 & 1.481952 & -1.122733 \\
$\mathrm{C}$ & 0.084607 & 2.819201 & 0.351826 \\
$\mathrm{C}$ & -1.630676 & 1.138936 & 1.779209 \\
$\mathrm{C}$ & -2.906117 & 0.403021 & -1.621941 \\
$\mathrm{C}$ & -2.317950 & 2.770480 & -1.667730 \\
$\mathrm{C}$ & -0.566212 & 3.942639 & 0.901826 \\
$\mathrm{C}$ & 1.398766 & 2.898180 & 0.052015 \\
$\mathrm{C}$ & -0.875112 & 1.071242 & 2.896397 \\
-3.025509 & 1.287708 & 1.892316 \\
-3.824390 & 0.662469 & -2.653425
\end{tabular}




\begin{tabular}{rrrr}
$\mathrm{C}$ & -2.697273 & -1.023354 & -1.260461 \\
$\mathrm{C}$ & -3.236630 & 2.998032 & -2.617522 \\
$\mathrm{H}$ & -1.725149 & 3.611277 & -1.353865 \\
$\mathrm{H}$ & -1.612292 & 3.899250 & 1.176650 \\
$\mathrm{C}$ & 0.146485 & 5.133356 & 1.082743 \\
$\mathrm{C}$ & 2.081340 & 4.047806 & 0.225844 \\
$\mathrm{H}$ & 1.923325 & 2.032090 & -0.328852 \\
$\mathrm{H}$ & 0.203010 & 1.037425 & 2.823982 \\
$\mathrm{C}$ & -1.458889 & 1.078385 & 4.168843 \\
$\mathrm{C}$ & -3.628761 & 1.329311 & 3.154262 \\
$\mathrm{H}$ & -3.648214 & 1.400989 & 1.017192 \\
$\mathrm{C}$ & -4.006517 & 1.957269 & -3.128778 \\
$\mathrm{H}$ & -4.376716 & -0.144421 & -3.117325 \\
$\mathrm{C}$ & -3.621748 & -1.751406 & -0.456966 \\
$\mathrm{C}$ & -1.692373 & -1.692954 & -1.868915 \\
$\mathrm{H}$ & -3.366153 & 3.998296 & -3.011633 \\
$\mathrm{C}$ & 1.445091 & 5.185557 & 0.746617 \\
$\mathrm{H}$ & -0.346974 & 6.005315 & 1.492639 \\
$\mathrm{H}$ & 3.130865 & 4.096337 & -0.032066 \\
$\mathrm{C}$ & -2.842490 & 1.205689 & 4.299606 \\
$\mathrm{H}$ & -0.835436 & 1.009258 & 5.050840 \\
$\mathrm{H}$ & -4.699228 & 1.459699 & 3.243116 \\
$\mathrm{H}$ & -4.722556 & 2.149545 & -3.917372 \\
$\mathrm{C}$ & -3.441782 & -3.139322 & -0.307075 \\
$\mathrm{~N}$ & -4.724709 & -1.113769 & 0.215809 \\
$\mathrm{C}$ & -1.529948 & -3.075488 & -1.704931 \\
$\mathrm{H}$ & -1.019251 & -1.178946 & -2.537356 \\
$\mathrm{H}$ & 2.004499 & 6.102179 & 0.883593 \\
$\mathrm{H}$ & -3.298815 & 1.228296 & 5.280632 \\
$\mathrm{C}$ & -2.379305 & -3.779826 & -0.946103 \\
$\mathrm{H}$ & -4.127359 & -3.724002 & 0.292164 \\
$\mathrm{C}$ & -4.925162 & -1.376832 & 1.642148 \\
$\mathrm{C}$ & -5.885139 & -0.666466 & -0.555792 \\
$\mathrm{H}$ & -0.725627 & -3.583742 & -2.216875 \\
$\mathrm{H}$ & -2.247319 & -4.848422 & -0.833957 \\
$\mathrm{H}$ & -5.517092 & -0.558264 & 2.101235 \\
$\mathrm{H}$ & -5.479903 & -2.329110 & 1.776527 \\
$\mathrm{H}$ & -3.947183 & -1.450695 & 2.164642 \\
$\mathrm{H}$ & -6.822473 & -1.073746 & -0.119394 \\
-5.934099 & 0.442225 & -0.542347 \\
$\mathrm{H}$ & -5.833377 & -1.028805 & -1.602311 \\
$\mathrm{H}$ & 1.630096 & -1.555219 & -2.955928 \\
$\mathrm{H}$ & -4.050815 & -2.856416 \\
$\mathrm{H}$ & -5.527281 & -0.863186 \\
\hline
\end{tabular}

Table S23: Cartesian coordinates $(\AA)$ of t2eTS $(\mathrm{E}=$ 1387.956208741113 a.u.).

\begin{tabular}{lrrr}
\hline \hline $\mathrm{C}$ & -0.149160 & -2.813883 & 2.880204 \\
$\mathrm{C}$ & -0.041871 & -2.091520 & 1.686923 \\
$\mathrm{C}$ & 0.922666 & -1.090264 & 1.511318
\end{tabular}




\begin{tabular}{|c|c|c|c|}
\hline $\mathrm{C}$ & 1.815397 & -0.829488 & 2.569903 \\
\hline $\mathrm{C}$ & 1.693726 & -1.550786 & 3.767375 \\
\hline $\mathrm{C}$ & 0.719222 & -2.536089 & 3.934118 \\
\hline $\mathrm{Pd}$ & 0.946220 & 0.019174 & -0.204722 \\
\hline $\mathrm{C}$ & 2.945594 & 0.152924 & 2.391341 \\
\hline $\mathrm{C}$ & 4.153249 & -0.497298 & 1.674273 \\
\hline $\mathrm{C}$ & 3.812787 & -1.308828 & 0.442884 \\
\hline $\mathrm{C}$ & 2.998966 & -0.755778 & -0.590557 \\
\hline $\mathrm{C}$ & 2.852322 & -1.508109 & -1.780938 \\
\hline $\mathrm{C}$ & 3.424857 & -2.770586 & -1.947070 \\
\hline $\mathrm{C}$ & 4.175821 & -3.277718 & -0.896324 \\
\hline $\mathrm{C}$ & 4.391851 & -2.569361 & 0.281560 \\
\hline $\mathrm{H}$ & -0.751253 & -2.296601 & 0.896584 \\
\hline $\mathrm{H}$ & 5.017777 & -3.009141 & 1.053600 \\
\hline $\mathrm{H}$ & 2.382976 & -1.330779 & 4.582187 \\
\hline $\mathrm{H}$ & 0.640853 & -3.076759 & 4.875179 \\
\hline $\mathrm{H}$ & -0.918890 & -3.577262 & 2.981386 \\
\hline $\mathrm{H}$ & 3.332483 & 0.521138 & -0.778499 \\
\hline $\mathrm{H}$ & 3.289188 & 0.534772 & 3.361115 \\
\hline $\mathrm{H}$ & 2.625436 & 1.020481 & 1.810649 \\
\hline $\mathrm{H}$ & 4.698745 & -1.136208 & 2.381597 \\
\hline $\mathrm{H}$ & 4.816071 & 0.326540 & 1.375128 \\
\hline $\mathrm{Br}$ & 0.764897 & 1.493228 & -2.479780 \\
\hline $\mathrm{O}$ & 3.967290 & 1.582933 & -1.148644 \\
\hline $\mathrm{C}$ & 3.977522 & 2.610497 & -0.354849 \\
\hline $\mathrm{O}$ & 4.313358 & 2.673392 & 0.824408 \\
\hline $\mathrm{O}$ & 3.555000 & 3.765242 & -0.982187 \\
\hline $\mathrm{H}$ & 3.163130 & 3.447988 & -1.816020 \\
\hline $\mathrm{P}$ & -1.163785 & 1.014357 & 0.211364 \\
\hline $\mathrm{C}$ & -2.326205 & 0.988771 & -1.223393 \\
\hline $\mathrm{C}$ & -0.845083 & 2.762792 & 0.633222 \\
\hline $\mathrm{C}$ & -2.128340 & 0.538109 & 1.695999 \\
\hline $\mathrm{C}$ & -2.700693 & -0.227266 & -1.870127 \\
\hline $\mathrm{C}$ & -2.824042 & 2.205799 & -1.742191 \\
\hline $\mathrm{C}$ & -1.875736 & 3.597441 & 1.111064 \\
\hline $\mathrm{C}$ & 0.418162 & 3.233652 & 0.585427 \\
\hline $\mathrm{C}$ & -1.519265 & 0.545997 & 2.901030 \\
\hline $\mathrm{C}$ & -3.516538 & 0.318595 & 1.620080 \\
\hline $\mathrm{C}$ & -3.522929 & -0.160481 & -3.007434 \\
\hline $\mathrm{C}$ & -2.117869 & -1.554297 & -1.542759 \\
\hline $\mathrm{C}$ & -3.646367 & 2.236752 & -2.801003 \\
\hline $\mathrm{H}$ & -2.539186 & 3.152934 & -1.319095 \\
\hline $\mathrm{H}$ & -2.892536 & 3.234856 & 1.189996 \\
\hline $\mathrm{C}$ & -1.580059 & 4.914842 & 1.480425 \\
\hline $\mathrm{C}$ & 0.700965 & 4.501207 & 0.946037 \\
\hline $\mathrm{H}$ & 1.222892 & 2.586913 & 0.265754 \\
\hline $\mathrm{H}$ & -0.468054 & 0.787254 & 2.979090 \\
\hline $\mathrm{C}$ & -2.231776 & 0.275047 & 4.075412 \\
\hline $\mathrm{C}$ & -4.255368 & 0.078040 & 2.783705 \\
\hline $\mathrm{H}$ & -4.038346 & 0.360157 & 0.675200 \\
\hline $\mathrm{C}$ & -4.014517 & 1.062835 & -3.452686 \\
\hline
\end{tabular}




\begin{tabular}{lrrr}
$\mathrm{H}$ & -3.754885 & -1.052570 & -3.574682 \\
$\mathrm{C}$ & -2.861809 & -2.577583 & -0.887999 \\
$\mathrm{C}$ & -0.895008 & -1.849555 & -2.038107 \\
$\mathrm{H}$ & -4.009588 & 3.187029 & -3.172052 \\
$\mathrm{C}$ & -0.315516 & 5.358910 & 1.395936 \\
$\mathrm{H}$ & -2.365721 & 5.568690 & 1.836733 \\
$\mathrm{H}$ & 1.721050 & 4.860116 & 0.898798 \\
$\mathrm{C}$ & -3.606107 & 0.038421 & 4.017582 \\
$\mathrm{H}$ & -1.721255 & 0.272160 & 5.029734 \\
$\mathrm{H}$ & -5.325026 & -0.075992 & 2.728151 \\
$\mathrm{H}$ & -4.653783 & 1.104983 & -4.325106 \\
$\mathrm{C}$ & -2.286597 & -3.856182 & -0.762019 \\
$\mathrm{~N}$ & -4.173555 & -2.343530 & -0.339391 \\
$\mathrm{C}$ & -0.339759 & -3.129380 & -1.900497 \\
$\mathrm{H}$ & -0.338451 & -1.109649 & -2.593392 \\
$\mathrm{H}$ & -0.076979 & 6.374904 & 1.683566 \\
$\mathrm{H}$ & -4.165671 & -0.155678 & 4.923325 \\
$\mathrm{C}$ & -1.014956 & -4.105503 & -1.280251 \\
$\mathrm{H}$ & -2.824423 & -4.658934 & -0.274971 \\
$\mathrm{C}$ & -4.449046 & -2.769389 & 1.034233 \\
$\mathrm{C}$ & -5.318262 & -2.179916 & -1.236509 \\
$\mathrm{H}$ & 0.634234 & -3.338519 & -2.320102 \\
$\mathrm{H}$ & -0.578135 & -5.091584 & -1.186796 \\
$\mathrm{H}$ & -5.309182 & -2.201139 & 1.444763 \\
$\mathrm{H}$ & -4.702844 & -3.850058 & 1.051271 \\
$\mathrm{H}$ & -3.561631 & -2.594781 & 1.679974 \\
$\mathrm{H}$ & -6.139124 & -2.871589 & -0.948992 \\
$\mathrm{H}$ & -5.689851 & -1.135822 & -1.176547 \\
$\mathrm{H}$ & -5.044158 & -2.423107 & -2.282804 \\
$\mathrm{H}$ & 2.290351 & -1.068644 & -2.600625 \\
$\mathrm{H}$ & 3.306437 & -3.349355 & -2.858408 \\
$\mathrm{~F}$ & 4.743878 & -4.503679 & -1.026840 \\
\hline & & & \\
\hline & & & \\
\hline
\end{tabular}

Table S24: Cartesian coordinates $(\AA)$ of $\mathbf{t 2 f R}(\mathrm{E}=$ 1387.968724024271 a.u.).

\begin{tabular}{lrrr}
\hline \hline $\mathrm{C}$ & -0.521190 & -3.065963 & 2.525339 \\
$\mathrm{C}$ & -0.430808 & -2.263008 & 1.384130 \\
$\mathrm{C}$ & 0.702950 & -1.485395 & 1.121724 \\
$\mathrm{C}$ & 1.775791 & -1.497591 & 2.037057 \\
$\mathrm{C}$ & 1.664088 & -2.304424 & 3.182422 \\
$\mathrm{C}$ & 0.534130 & -3.081765 & 3.435126 \\
$\mathrm{Pd}$ & 0.734667 & -0.316474 & -0.559465 \\
$\mathrm{C}$ & 3.050193 & -0.725733 & 1.795145 \\
$\mathrm{C}$ & 4.055510 & -1.520344 & 0.910747 \\
$\mathrm{C}$ & 3.378611 & -2.111280 & -0.292239 \\
$\mathrm{C}$ & 2.881688 & -1.268092 & -1.319908 \\
$\mathrm{C}$ & 2.201981 & -1.814841 & -2.428733 \\
$\mathrm{C}$ & 2.015092 & -3.199323 & -2.530352 \\
$\mathrm{C}$ & 2.500002 & -4.035223 & -1.529209 \\
$\mathrm{C}$ & 3.157969 & -3.476823 & -0.434696
\end{tabular}




\begin{tabular}{|c|c|c|c|}
\hline $\mathrm{H}$ & -1.273035 & -2.229622 & 0.707691 \\
\hline $\mathrm{H}$ & 2.491415 & -2.311561 & 3.890279 \\
\hline $\mathrm{H}$ & 0.478306 & -3.687678 & 4.336969 \\
\hline $\mathrm{H}$ & -1.417615 & -3.658015 & 2.699880 \\
\hline $\mathrm{H}$ & 3.231735 & -0.229025 & -1.312540 \\
\hline $\mathrm{H}$ & 3.546305 & -0.487445 & 2.741886 \\
\hline $\mathrm{H}$ & 2.872891 & 0.238214 & 1.312219 \\
\hline $\mathrm{H}$ & 4.521930 & -2.315875 & 1.499820 \\
\hline $\mathrm{H}$ & 4.806671 & -0.797535 & 0.568911 \\
\hline $\mathrm{Br}$ & 0.631673 & 1.315117 & -2.701043 \\
\hline $\mathrm{O}$ & 4.948476 & 0.960316 & -0.778388 \\
\hline $\mathrm{C}$ & 5.028179 & 1.861799 & 0.107752 \\
\hline $\mathrm{O}$ & 4.650056 & 1.877029 & 1.289593 \\
\hline $\mathrm{O}$ & 5.669162 & 3.045574 & -0.337894 \\
\hline $\mathrm{H}$ & 5.855830 & 2.837115 & -1.267884 \\
\hline $\mathrm{P}$ & -0.822812 & 1.164683 & 0.293984 \\
\hline $\mathrm{C}$ & -2.184868 & 1.572087 & -0.885141 \\
\hline $\mathrm{C}$ & 0.054554 & 2.719921 & 0.682275 \\
\hline C & -1.596750 & 0.838540 & 1.923030 \\
\hline $\mathrm{C}$ & -2.986154 & 0.563787 & -1.503111 \\
\hline $\mathrm{C}$ & -2.416698 & 2.921375 & -1.238342 \\
\hline $\mathrm{C}$ & -0.584352 & 3.746367 & 1.407895 \\
\hline C & 1.354258 & 2.860265 & 0.344538 \\
\hline $\mathrm{C}$ & -0.802443 & 0.632761 & 2.995576 \\
\hline $\mathrm{C}$ & -2.988327 & 0.950021 & 2.101293 \\
\hline $\mathrm{C}$ & -3.940011 & 0.953120 & -2.458730 \\
\hline $\mathrm{C}$ & -2.756279 & -0.896546 & -1.350775 \\
\hline C & -3.368168 & 3.267701 & -2.117240 \\
\hline $\mathrm{H}$ & -1.820054 & 3.717630 & -0.829995 \\
\hline $\mathrm{H}$ & -1.617856 & 3.649280 & 1.714671 \\
\hline $\mathrm{C}$ & 0.122840 & 4.911871 & 1.723518 \\
\hline $\mathrm{C}$ & 2.031263 & 3.986064 & 0.648063 \\
\hline $\mathrm{H}$ & 1.871494 & 2.063496 & -0.173024 \\
\hline $\mathrm{H}$ & 0.272727 & 0.626745 & 2.884276 \\
\hline $\mathrm{C}$ & -1.341863 & 0.459629 & 4.275853 \\
\hline $\mathrm{C}$ & -3.547943 & 0.812389 & 3.376489 \\
\hline $\mathrm{H}$ & -3.642216 & 1.171435 & 1.270797 \\
\hline $\mathrm{C}$ & -4.146978 & 2.299078 & -2.743557 \\
\hline $\mathrm{H}$ & -4.501892 & 0.211916 & -3.012258 \\
\hline $\mathrm{C}$ & -3.645819 & -1.738643 & -0.622586 \\
\hline $\mathrm{C}$ & -1.773598 & -1.464929 & -2.085370 \\
\hline $\mathrm{H}$ & -3.517799 & 4.311396 & -2.364120 \\
\hline $\mathrm{C}$ & 1.405547 & 5.030786 & 1.346158 \\
\hline $\mathrm{H}$ & -0.362216 & 5.710964 & 2.269334 \\
\hline $\mathrm{H}$ & 3.068214 & 4.087222 & 0.357141 \\
\hline $\mathrm{C}$ & -2.721306 & 0.547400 & 4.468105 \\
\hline $\mathrm{H}$ & -0.687529 & 0.282096 & 5.119477 \\
\hline $\mathrm{H}$ & -4.615928 & 0.914075 & 3.517416 \\
\hline $\mathrm{H}$ & -4.890003 & 2.590490 & -3.474663 \\
\hline $\mathrm{C}$ & -3.454356 & -3.132158 & -0.675852 \\
\hline $\mathrm{N}$ & -4.724892 & -1.212059 & 0.173891 \\
\hline
\end{tabular}




\begin{tabular}{lrrr}
$\mathrm{C}$ & -1.599599 & -2.855148 & -2.123555 \\
$\mathrm{H}$ & -1.129832 & -0.856225 & -2.701178 \\
$\mathrm{H}$ & 1.960538 & 5.928769 & 1.585859 \\
$\mathrm{H}$ & -3.143582 & 0.431107 & 5.457741 \\
$\mathrm{C}$ & -2.415419 & -3.666808 & -1.438814 \\
$\mathrm{H}$ & -4.113644 & -3.801732 & -0.139248 \\
$\mathrm{C}$ & -4.873473 & -1.679030 & 1.553689 \\
$\mathrm{C}$ & -5.912286 & -0.663562 & -0.482870 \\
$\mathrm{H}$ & -0.814853 & -3.279046 & -2.733192 \\
$\mathrm{H}$ & -2.275144 & -4.739309 & -1.483201 \\
$\mathrm{H}$ & -5.453831 & -0.941455 & 2.145557 \\
$\mathrm{H}$ & -5.417597 & -2.646646 & 1.568783 \\
$\mathrm{H}$ & -3.877194 & -1.815928 & 2.026552 \\
$\mathrm{H}$ & -6.833518 & -1.132820 & -0.075398 \\
$\mathrm{H}$ & -5.959321 & 0.431597 & -0.309045 \\
$\mathrm{H}$ & -5.899479 & -0.872302 & -1.571592 \\
$\mathrm{H}$ & 1.870324 & -1.151924 & -3.219946 \\
$\mathrm{H}$ & 1.513998 & -3.623405 & -3.396238 \\
$\mathrm{~F}$ & 3.606952 & -4.329223 & 0.523220 \\
$\mathrm{H}$ & 2.375061 & -5.113442 & -1.571371 \\
\hline \hline
\end{tabular}

Table S25: Cartesian coordinates $(\AA)$ of t2fTS $(\mathrm{E}=$ 1387.953798687830 a.u.).

\begin{tabular}{lrrr}
\hline \hline $\mathrm{C}$ & -0.085664 & -3.149864 & 2.486649 \\
$\mathrm{C}$ & -0.029031 & -2.292202 & 1.382871 \\
$\mathrm{C}$ & 0.954634 & -1.301327 & 1.267103 \\
$\mathrm{C}$ & 1.917852 & -1.187922 & 2.288873 \\
$\mathrm{C}$ & 1.846140 & -2.045477 & 3.397412 \\
$\mathrm{C}$ & 0.853935 & -3.020729 & 3.507712 \\
$\mathrm{Pd}$ & 0.921380 & -0.004356 & -0.311266 \\
$\mathrm{C}$ & 3.067149 & -0.219782 & 2.157642 \\
$\mathrm{C}$ & 4.219079 & -0.819958 & 1.315366 \\
$\mathrm{C}$ & 3.781643 & -1.475157 & 0.024377 \\
$\mathrm{C}$ & 2.935201 & -0.803136 & -0.903014 \\
$\mathrm{C}$ & 2.676876 & -1.415753 & -2.151189 \\
$\mathrm{C}$ & 3.185073 & -2.676700 & -2.471559 \\
$\mathrm{C}$ & 3.987541 & -3.344223 & -1.547985 \\
$\mathrm{C}$ & 4.276935 & -2.725119 & -0.334739 \\
$\mathrm{H}$ & -0.790272 & -2.383936 & 0.619764 \\
$\mathrm{H}$ & 2.591110 & -1.943023 & 4.185792 \\
$\mathrm{H}$ & 0.817206 & -3.669231 & 4.380673 \\
$\mathrm{H}$ & -0.870771 & -3.901856 & 2.545910 \\
$\mathrm{H}$ & 3.271988 & 0.474943 & -0.956074 \\
$\mathrm{H}$ & 3.468680 & 0.044627 & 3.144088 \\
$\mathrm{H}$ & 2.745141 & 0.714166 & 1.692339 \\
$\mathrm{H}$ & 4.772839 & -1.548634 & 1.915061 \\
$\mathrm{H}$ & 4.893749 & 0.012021 & 1.070756 \\
$\mathrm{Br}$ & 0.657885 & 1.736212 & -2.380010 \\
$\mathrm{O}$ & 3.935048 & 1.557833 & -1.246731 \\
$\mathrm{C}$ & 4.015845 & 2.498619 & -0.355356
\end{tabular}




\begin{tabular}{|c|c|c|c|}
\hline $\mathrm{O}$ & 4.440370 & 2.439093 & 0.795192 \\
\hline C & 3.558343 & 3.712991 & -0.827220 \\
\hline $\mathrm{H}$ & 3.095149 & 3.482357 & -1.653391 \\
\hline $\mathrm{P}$ & -1.118797 & 0.993566 & 0.352031 \\
\hline $\mathrm{C}$ & -2.364368 & 1.174786 & -0.999289 \\
\hline $\mathrm{C}$ & -0.713217 & 2.668282 & 0.957739 \\
\hline $\mathrm{C}$ & -2.010608 & 0.370974 & 1.828163 \\
\hline C & -2.821988 & 0.056851 & -1.759614 \\
\hline $\mathrm{C}$ & -2.845567 & 2.459784 & -1.339102 \\
\hline $\mathrm{C}$ & -1.683596 & 3.468696 & 1.594160 \\
\hline $\mathrm{C}$ & 0.561421 & 3.103975 & 0.889417 \\
\hline $\mathrm{C}$ & -1.333224 & 0.223213 & 2.986919 \\
\hline $\mathrm{C}$ & -3.407318 & 0.197741 & 1.812810 \\
\hline $\mathrm{C}$ & -3.705982 & 0.284483 & -2.827698 \\
\hline $\mathrm{C}$ & -2.271550 & -1.316997 & -1.627638 \\
\hline $\mathrm{C}$ & -3.726824 & 2.642342 & -2.333213 \\
\hline $\mathrm{H}$ & -2.500669 & 3.340409 & -0.826484 \\
\hline $\mathrm{H}$ & -2.706754 & 3.129186 & 1.691507 \\
\hline $\mathrm{C}$ & -1.319157 & 4.722038 & 2.099852 \\
\hline $\mathrm{C}$ & 0.910838 & 4.309263 & 1.381295 \\
\hline $\mathrm{H}$ & 1.321743 & 2.477024 & 0.446188 \\
\hline $\mathrm{H}$ & -0.272263 & 0.427895 & 3.028264 \\
\hline $\mathrm{C}$ & -1.985051 & -0.164778 & 4.163691 \\
\hline $\mathrm{C}$ & -4.085119 & -0.157819 & 2.984129 \\
\hline $\mathrm{H}$ & -3.981400 & 0.363350 & 0.912967 \\
\hline $\mathrm{C}$ & -4.176386 & 1.566706 & -3.094221 \\
\hline $\mathrm{H}$ & -4.003927 & -0.525653 & -3.480592 \\
\hline $\mathrm{C}$ & -3.013722 & -2.388397 & -1.052378 \\
\hline $\mathrm{C}$ & -1.091856 & -1.587760 & -2.230061 \\
\hline $\mathrm{H}$ & -4.075029 & 3.640852 & -2.566395 \\
\hline $\mathrm{C}$ & -0.046044 & 5.135497 & 1.992027 \\
\hline $\mathrm{H}$ & -2.058601 & 5.350498 & 2.579432 \\
\hline $\mathrm{H}$ & 1.939106 & 4.640823 & 1.315330 \\
\hline $\mathrm{C}$ & -3.367313 & -0.357974 & 4.163173 \\
\hline $\mathrm{H}$ & -1.420652 & -0.291717 & 5.078412 \\
\hline $\mathrm{H}$ & -5.160707 & -0.276191 & 2.977010 \\
\hline $\mathrm{H}$ & -4.863479 & 1.732516 & -3.913955 \\
\hline $\mathrm{C}$ & -2.479955 & -3.689537 & -1.113349 \\
\hline $\mathrm{N}$ & -4.282355 & -2.182741 & -0.400472 \\
\hline $\mathrm{C}$ & -0.578142 & -2.891106 & -2.279351 \\
\hline $\mathrm{H}$ & -0.541399 & -0.803915 & -2.728313 \\
\hline $\mathrm{H}$ & 0.245754 & 6.101339 & 2.384303 \\
\hline $\mathrm{H}$ & -3.879643 & -0.641949 & 5.073138 \\
\hline $\mathrm{C}$ & -1.251419 & -3.913421 & -1.736385 \\
\hline $\mathrm{H}$ & -3.017847 & -4.528290 & -0.691416 \\
\hline $\mathrm{C}$ & -4.495475 & -2.764772 & 0.926187 \\
\hline $\mathrm{C}$ & -5.469309 & -1.875696 & -1.199775 \\
\hline $\mathrm{H}$ & 0.360286 & -3.078119 & -2.781992 \\
\hline $\mathrm{H}$ & -0.847223 & -4.916381 & -1.787359 \\
\hline $\mathrm{H}$ & -5.308161 & -2.224249 & 1.454032 \\
\hline $\mathrm{H}$ & -4.789726 & -3.830804 & 0.828473 \\
\hline
\end{tabular}




\begin{tabular}{lrrr}
$\mathrm{H}$ & -3.566629 & -2.698763 & 1.532703 \\
$\mathrm{H}$ & -6.299232 & -2.569571 & -0.945482 \\
$\mathrm{H}$ & -5.795132 & -0.834734 & -0.994728 \\
$\mathrm{H}$ & -5.265631 & -2.001023 & -2.282263 \\
$\mathrm{H}$ & 2.087288 & -0.869459 & -2.881976 \\
$\mathrm{H}$ & 2.968903 & -3.135995 & -3.433787 \\
$\mathrm{~F}$ & 5.101082 & -3.399065 & 0.520198 \\
$\mathrm{H}$ & 4.411705 & -4.323440 & -1.752137 \\
\hline \hline
\end{tabular}

Table S26: Cartesian coordinates $(\AA)$ of t2gR $(\mathrm{E}=$ 1487.189319426490 a.u.).

\begin{tabular}{lrrr}
\hline \hline $\mathrm{C}$ & -0.890328 & -1.899049 & 3.553193 \\
$\mathrm{C}$ & -0.680745 & -1.540007 & 2.217447 \\
$\mathrm{C}$ & 0.495340 & -0.901271 & 1.807959 \\
$\mathrm{C}$ & 1.479426 & -0.589988 & 2.767808 \\
$\mathrm{C}$ & 1.250384 & -0.947705 & 4.106394 \\
$\mathrm{C}$ & 0.080864 & -1.595898 & 4.505735 \\
$\mathrm{Pd}$ & 0.780677 & -0.357761 & -0.143999 \\
$\mathrm{C}$ & 2.787231 & 0.041881 & 2.367317 \\
$\mathrm{C}$ & 3.808775 & -1.019248 & 1.873115 \\
$\mathrm{C}$ & 3.234974 & -2.001518 & 0.885690 \\
$\mathrm{C}$ & 3.024589 & -1.607927 & -0.462910 \\
$\mathrm{C}$ & 2.620290 & -2.580645 & -1.391917 \\
$\mathrm{C}$ & 2.346548 & -3.886500 & -0.990061 \\
$\mathrm{C}$ & 2.495084 & -4.257422 & 0.337830 \\
$\mathrm{C}$ & 2.940238 & -3.317054 & 1.267283 \\
$\mathrm{H}$ & -1.466676 & -1.739707 & 1.501910 \\
$\mathrm{H}$ & 3.079100 & -3.614514 & 2.302708 \\
$\mathrm{H}$ & 2.010942 & -0.701096 & 4.846000 \\
$\mathrm{H}$ & -0.072791 & -1.850864 & 5.552230 \\
$\mathrm{H}$ & -1.815267 & -2.395846 & 3.839953 \\
$\mathrm{H}$ & 3.445572 & -0.652420 & -0.797667 \\
$\mathrm{H}$ & 3.238456 & 0.582520 & 3.205968 \\
$\mathrm{H}$ & 2.670440 & 0.786361 & 1.575234 \\
$\mathrm{H}$ & 4.192512 & -1.577800 & 2.737288 \\
$\mathrm{H}$ & 4.621615 & -0.465958 & 1.385715 \\
$\mathrm{Br}$ & 0.983353 & 0.492211 & -2.661151 \\
$\mathrm{O}$ & 5.023610 & 0.678629 & -0.453825 \\
$\mathrm{C}$ & 4.970087 & 1.848551 & 0.030597 \\
$\mathrm{O}$ & 4.514385 & 2.259888 & 1.109215 \\
$\mathrm{O}$ & 5.537958 & 2.840093 & -0.805017 \\
$\mathrm{H}$ & 5.795507 & 2.325739 & -1.587497 \\
$\mathrm{~F}$ & 2.568870 & -2.313366 & -2.699372 \\
$\mathrm{P}$ & -0.847160 & 1.267486 & -0.057782 \\
$\mathrm{C}$ & -2.035991 & 1.196155 & -1.468501 \\
$\mathrm{C}$ & -0.021464 & 2.896957 & -0.101297 \\
$\mathrm{C}$ & -1.835650 & 1.474142 & 1.471986 \\
$\mathrm{C}$ & -2.760318 & 0.009071 & -1.795719 \\
$\mathrm{C}$ & -2.198225 & 2.330194 & -2.296955 \\
$\mathrm{C}$ & -0.752375 & 4.080663 & 0.127987
\end{tabular}




\begin{tabular}{|c|c|c|c|}
\hline $\mathrm{C}$ & 1.316857 & 2.968598 & -0.265112 \\
\hline $\mathrm{C}$ & -1.193243 & 1.675004 & 2.643023 \\
\hline $\mathrm{C}$ & -3.238167 & 1.578715 & 1.422974 \\
\hline $\mathrm{C}$ & -3.568809 & 0.009402 & -2.944883 \\
\hline $\mathrm{C}$ & -2.578614 & -1.295865 & -1.106987 \\
\hline $\mathrm{C}$ & -3.016995 & 2.317302 & -3.358677 \\
\hline $\mathrm{H}$ & -1.649181 & 3.237618 & -2.117210 \\
\hline $\mathrm{H}$ & -1.822091 & 4.049817 & 0.290838 \\
\hline $\mathrm{C}$ & -0.087878 & 5.312258 & 0.134182 \\
\hline $\mathrm{C}$ & 1.953474 & 4.157061 & -0.258922 \\
\hline $\mathrm{H}$ & 1.897849 & 2.066244 & -0.398259 \\
\hline $\mathrm{H}$ & -0.112470 & 1.673829 & 2.679139 \\
\hline $\mathrm{C}$ & -1.900378 & 1.919677 & 3.826221 \\
\hline C & -3.964428 & 1.854069 & 2.587100 \\
\hline $\mathrm{H}$ & -3.773790 & 1.479329 & 0.490389 \\
\hline C & -3.716637 & 1.165114 & -3.705379 \\
\hline $\mathrm{H}$ & -4.057615 & -0.896775 & -3.277032 \\
\hline $\mathrm{C}$ & -3.594264 & -1.877329 & -0.294182 \\
\hline $\mathrm{C}$ & -1.482978 & -2.029443 & -1.406443 \\
\hline $\mathrm{H}$ & -3.116722 & 3.204404 & -3.971683 \\
\hline $\mathrm{C}$ & 1.239898 & 5.349388 & -0.059995 \\
\hline $\mathrm{H}$ & -0.641787 & 6.227893 & 0.297116 \\
\hline $\mathrm{H}$ & 3.025200 & 4.195772 & -0.401861 \\
\hline $\mathrm{C}$ & -3.292823 & 2.007909 & 3.799690 \\
\hline $\mathrm{H}$ & -1.365972 & 2.063410 & 4.756364 \\
\hline $\mathrm{H}$ & -5.041254 & 1.951819 & 2.548283 \\
\hline $\mathrm{H}$ & -4.348739 & 1.158706 & -4.584016 \\
\hline $\mathrm{C}$ & -3.410302 & -3.186333 & 0.187704 \\
\hline $\mathrm{N}$ & -4.795577 & -1.166901 & 0.061678 \\
\hline $\mathrm{C}$ & -1.318462 & -3.331902 & -0.914948 \\
\hline $\mathrm{H}$ & -0.728868 & -1.639374 & -2.072070 \\
\hline $\mathrm{H}$ & 1.762518 & 6.297416 & -0.055575 \\
\hline $\mathrm{H}$ & -3.844755 & 2.213425 & 4.707638 \\
\hline $\mathrm{C}$ & -2.254277 & -3.895706 & -0.140343 \\
\hline $\mathrm{H}$ & -4.163800 & -3.657660 & 0.805372 \\
\hline $\mathrm{C}$ & -5.171248 & -1.086836 & 1.474159 \\
\hline $\mathrm{C}$ & -5.863067 & -1.006638 & -0.926706 \\
\hline $\mathrm{H}$ & -0.437498 & -3.896436 & -1.181838 \\
\hline $\mathrm{H}$ & -2.117841 & -4.904845 & 0.227007 \\
\hline $\mathrm{H}$ & -5.802292 & -0.190895 & 1.648283 \\
\hline $\mathrm{H}$ & -5.750704 & -1.989114 & 1.762164 \\
\hline $\mathrm{H}$ & -4.266202 & -1.021798 & 2.115534 \\
\hline $\mathrm{H}$ & -6.831504 & -1.363301 & -0.514549 \\
\hline $\mathrm{H}$ & -5.960700 & 0.065573 & -1.196030 \\
\hline $\mathrm{H}$ & -5.659638 & -1.607301 & -1.836336 \\
\hline $\mathrm{H}$ & 2.274831 & -5.282133 & 0.622746 \\
\hline $\mathrm{F}$ & 1.972863 & -4.797991 & -1.916491 \\
\hline
\end{tabular}


Table S27: Cartesian coordinates $(\AA)$ of t2gTS $(\mathrm{E}=$ 1487.179848801932 a.u.).

\begin{tabular}{lrrr}
\hline \hline $\mathrm{C}$ & -0.399036 & -2.455953 & 3.216369 \\
$\mathrm{C}$ & -0.215605 & -1.891403 & 1.949267 \\
$\mathrm{C}$ & 0.767223 & -0.922981 & 1.710796 \\
$\mathrm{C}$ & 1.592668 & -0.521548 & 2.779981 \\
$\mathrm{C}$ & 1.394517 & -1.086225 & 4.048792 \\
$\mathrm{C}$ & 0.406216 & -2.045397 & 4.277094 \\
$\mathrm{Pd}$ & 0.934162 & -0.030604 & -0.121209 \\
$\mathrm{C}$ & 2.731565 & 0.439248 & 2.544342 \\
$\mathrm{C}$ & 3.987625 & -0.290773 & 2.004330 \\
$\mathrm{C}$ & 3.709455 & -1.333362 & 0.944288 \\
$\mathrm{C}$ & 3.024536 & -0.974222 & -0.258061 \\
$\mathrm{C}$ & 2.938099 & -1.963292 & -1.246951 \\
$\mathrm{C}$ & 3.424199 & -3.257487 & -1.060865 \\
$\mathrm{C}$ & 4.045217 & -3.601584 & 0.126391 \\
$\mathrm{C}$ & 4.195215 & -2.629760 & 1.121904 \\
$\mathrm{H}$ & -0.876085 & -2.196519 & 1.148868 \\
$\mathrm{H}$ & 4.707003 & -2.891761 & 2.044919 \\
$\mathrm{H}$ & 2.032265 & -0.762363 & 4.870789 \\
$\mathrm{H}$ & 0.266980 & -2.462526 & 5.272346 \\
$\mathrm{H}$ & -1.178379 & -3.200956 & 3.367175 \\
$\mathrm{H}$ & 3.373529 & 0.203791 & -0.657430 \\
$\mathrm{H}$ & 3.004457 & 0.959189 & 3.471251 \\
$\mathrm{H}$ & 2.448660 & 1.211321 & 1.825139 \\
$\mathrm{H}$ & 4.510356 & -0.775252 & 2.839464 \\
$\mathrm{H}$ & 4.642536 & 0.485808 & 1.585814 \\
$\mathrm{Br}$ & 0.865989 & 1.293975 & -2.497144 \\
$\mathrm{O}$ & 4.073882 & 1.223820 & -1.152238 \\
$\mathrm{C}$ & 4.083532 & 2.350638 & -0.512267 \\
$\mathrm{O}$ & 4.442146 & 2.581087 & 0.642252 \\
$\mathrm{H}$ & -2.790491 & 3.470243 & 0.797024 \\
$\mathrm{C}$ & -1.420160 & 5.119245 & 0.977644 \\
$\mathrm{H}$ & 3.200103 & 2.979586 & -2.039845 \\
$\mathrm{~F}$ & 2.400950 & -1.697056 & -2.449167 \\
$\mathrm{P}$ & -1.124151 & 1.098459 & 0.115755 \\
$\mathrm{C}$ & -2.244854 & 0.970588 & -1.346064 \\
$\mathrm{C}$ & -0.745101 & 2.866742 & 0.376399 \\
$\mathrm{C}$ & -2.155334 & 0.813306 & 1.606615 \\
$\mathrm{C}$ & -2.656835 & -0.289195 & -1.876488 \\
$\mathrm{C}$ & -2.673768 & 2.147361 & -2.001504 \\
$\mathrm{C}$ & -1.756944 & 3.784060 & 0.725981 \\
$\mathrm{C}$ & 0.537878 & 3.281821 & 0.339017 \\
$\mathrm{C}$ & -1.595456 & 0.961975 & 2.826511 \\
$\mathrm{C}$ & -3.542698 & 0.600433 & 1.501337 \\
$\mathrm{H}$ & -2.444106 & -0.305014 & -3.040178 \\
$\mathrm{H}$ & -3.465726 & -1.599342 & -1.399104 \\
$\mathrm{H}$ & 2.103585 & -3.082648 \\
$\mathrm{H}$ & & & \\
$\mathrm{C}$ & -357055 & 3.120225 & -1.669147 \\
$\mathrm{H}$ & &
\end{tabular}




\begin{tabular}{rrrr}
$\mathrm{C}$ & 0.860284 & 4.566963 & 0.585633 \\
$\mathrm{H}$ & 1.326249 & 2.576807 & 0.119662 \\
$\mathrm{H}$ & -0.546491 & 1.206391 & 2.918434 \\
$\mathrm{C}$ & -2.357196 & 0.840218 & 3.994785 \\
$\mathrm{C}$ & -4.330197 & 0.508008 & 2.654359 \\
$\mathrm{H}$ & -4.027152 & 0.535438 & 0.538353 \\
$\mathrm{C}$ & -3.868640 & 0.885339 & -3.622482 \\
$\mathrm{H}$ & -3.698293 & -1.239599 & -3.522766 \\
$\mathrm{C}$ & -2.958176 & -2.532386 & -0.695721 \\
$\mathrm{C}$ & -0.91154 & -1.982462 & -1.803836 \\
$\mathrm{H}$ & -3.775771 & 3.024957 & -3.559703 \\
$\mathrm{C}$ & -0.135531 & 5.503717 & 0.904974 \\
$\mathrm{H}$ & -2.190624 & 5.834919 & 1.234415 \\
$\mathrm{H}$ & 1.896099 & 4.879262 & 0.547271 \\
$\mathrm{C}$ & -3.730952 & 0.610098 & 3.909595 \\
$\mathrm{H}$ & -1.885014 & 0.948774 & 4.962588 \\
$\mathrm{H}$ & -5.399202 & 0.360906 & 2.574347 \\
$\mathrm{H}$ & -4.481439 & 0.864093 & -4.514441 \\
$\mathrm{C}$ & -2.444251 & -3.815689 & -0.431744 \\
$\mathrm{~N}$ & -4.282273 & -2.202232 & -0.233567 \\
$\mathrm{C}$ & -0.418474 & -3.266640 & -1.530539 \\
$\mathrm{H}$ & -0.298455 & -1.313999 & -2.390547 \\
$\mathrm{H}$ & 0.134617 & 6.533299 & 1.102071 \\
$\mathrm{H}$ & -4.328608 & 0.531721 & 4.808388 \\
$\mathrm{C}$ & -1.162565 & -4.158378 & -0.863991 \\
$\mathrm{H}$ & -3.037741 & -4.550926 & 0.095704 \\
$\mathrm{C}$ & -4.628396 & -2.477829 & 1.162224 \\
$\mathrm{C}$ & -5.383140 & -2.105188 & -1.193092 \\
$\mathrm{H}$ & 0.563314 & -3.550606 & -1.881572 \\
$\mathrm{H}$ & -0.773041 & -5.148728 & -0.665480 \\
$\mathrm{H}$ & -5.478552 & -1.838306 & 1.477525 \\
$\mathrm{H}$ & -4.928354 & -3.540882 & 1.274424 \\
$\mathrm{H}$ & -3.759881 & -2.275076 & 1.825176 \\
$\mathrm{H}$ & -6.237743 & -2.737657 & -0.869698 \\
$\mathrm{H}$ & -5.722378 & -1.050737 & -1.262440 \\
$\mathrm{H}$ & -5.074509 & -2.469024 & -2.193845 \\
$\mathrm{H}$ & 4.416164 & -4.614558 & 0.254187 \\
$\mathrm{~F}$ & 3.291478 & -4.172910 & -2.050964 \\
\hline \hline & & & \\
\hline
\end{tabular}

Table S28: Cartesian coordinates $(\AA)$ of $\mathbf{t 2 h R}(\mathrm{E}=$ 1487.198063118640 a.u.).

\begin{tabular}{lrrr}
\hline \hline $\mathrm{C}$ & -0.682381 & -2.484682 & 3.138965 \\
$\mathrm{C}$ & -0.523840 & -1.911466 & 1.872612 \\
$\mathrm{C}$ & 0.599654 & -1.140306 & 1.554827 \\
$\mathrm{C}$ & 1.586587 & -0.924682 & 2.537991 \\
$\mathrm{C}$ & 1.408472 & -1.496860 & 3.807853 \\
$\mathrm{C}$ & 0.287775 & -2.269424 & 4.116112 \\
$\mathrm{Pd}$ & 0.789429 & -0.268990 & -0.286185 \\
$\mathrm{C}$ & 2.850947 & -0.168823 & 2.217260 \\
$\mathrm{C}$ & 3.905172 & -1.083269 & 1.531592
\end{tabular}




\begin{tabular}{|c|c|c|c|}
\hline C & 3.328899 & -1.945920 & 0.441696 \\
\hline $\mathrm{C}$ & 2.987866 & -1.368265 & -0.814301 \\
\hline $\mathrm{C}$ & 2.580155 & -2.239460 & -1.843274 \\
\hline $\mathrm{C}$ & 2.397648 & -3.605761 & -1.654649 \\
\hline $\mathrm{C}$ & 2.677925 & -4.108841 & -0.391379 \\
\hline $\mathrm{C}$ & 3.143592 & -3.316916 & 0.649286 \\
\hline $\mathrm{H}$ & -1.306094 & -2.054095 & 1.139784 \\
\hline $\mathrm{H}$ & 3.368613 & -3.772997 & 1.607353 \\
\hline $\mathrm{H}$ & 2.169760 & -1.322683 & 4.567079 \\
\hline $\mathrm{H}$ & 0.172166 & -2.691919 & 5.112020 \\
\hline $\mathrm{H}$ & -1.567495 & -3.079960 & 3.355220 \\
\hline $\mathrm{H}$ & 3.362575 & -0.363283 & -1.039737 \\
\hline $\mathrm{H}$ & 3.304005 & 0.248013 & 3.122904 \\
\hline $\mathrm{H}$ & 2.676994 & 0.687089 & 1.560391 \\
\hline $\mathrm{H}$ & 4.364955 & -1.733587 & 2.287069 \\
\hline $\mathrm{H}$ & 4.656970 & -0.416993 & 1.087204 \\
\hline $\mathrm{Br}$ & 0.863447 & 1.009037 & -2.625321 \\
\hline $\mathrm{O}$ & 4.958435 & 0.948911 & -0.560382 \\
\hline $\mathrm{C}$ & 4.872609 & 2.036233 & 0.085663 \\
\hline $\mathrm{O}$ & 4.479064 & 2.262652 & 1.240759 \\
\hline $\mathrm{O}$ & 5.311554 & 3.169252 & -0.639375 \\
\hline $\mathrm{H}$ & 5.536076 & 2.785861 & -1.502909 \\
\hline $\mathrm{F}$ & 2.425710 & -1.775146 & -3.087898 \\
\hline $\mathrm{P}$ & -0.906199 & 1.244542 & 0.127552 \\
\hline $\mathrm{C}$ & -2.143831 & 1.364529 & -1.237103 \\
\hline $\mathrm{C}$ & -0.147208 & 2.892923 & 0.341636 \\
\hline $\mathrm{C}$ & -1.846660 & 1.144931 & 1.698199 \\
\hline C & -2.829677 & 0.219572 & -1.746291 \\
\hline $\mathrm{C}$ & -2.388507 & 2.617453 & -1.844511 \\
\hline $\mathrm{C}$ & -0.913465 & 3.985167 & 0.797898 \\
\hline $\mathrm{C}$ & 1.179621 & 3.052345 & 0.148810 \\
\hline $\mathrm{C}$ & -1.171714 & 1.175592 & 2.867633 \\
\hline C & -3.253276 & 1.191614 & 1.707664 \\
\hline $\mathrm{C}$ & -3.686884 & 0.382935 & -2.847575 \\
\hline $\mathrm{C}$ & -2.556850 & -1.176662 & -1.315520 \\
\hline $\mathrm{C}$ & -3.250478 & 2.751759 & -2.862744 \\
\hline $\mathrm{H}$ & -1.871477 & 3.504905 & -1.524867 \\
\hline $\mathrm{H}$ & -1.973710 & 3.878732 & 0.988381 \\
\hline $\mathrm{C}$ & -0.297463 & 5.225885 & 0.996781 \\
\hline $\mathrm{C}$ & 1.769516 & 4.249308 & 0.341156 \\
\hline $\mathrm{H}$ & 1.789306 & 2.213964 & -0.158764 \\
\hline $\mathrm{H}$ & -0.091595 & 1.221071 & 2.872394 \\
\hline $\mathrm{C}$ & -1.845460 & 1.184438 & 4.094788 \\
\hline $\mathrm{C}$ & -3.948098 & 1.233204 & 2.921645 \\
\hline $\mathrm{H}$ & -3.817803 & 1.225988 & 0.787689 \\
\hline $\mathrm{C}$ & -3.915733 & 1.645998 & -3.384306 \\
\hline $\mathrm{H}$ & -4.151567 & -0.473705 & -3.317986 \\
\hline $\mathrm{C}$ & -3.495328 & -1.941937 & -0.564504 \\
\hline $\mathrm{C}$ & -1.460781 & -1.793124 & -1.812235 \\
\hline $\mathrm{H}$ & -3.413545 & 3.727076 & -3.304065 \\
\hline C & 1.018968 & 5.355844 & 0.768489 \\
\hline
\end{tabular}




\begin{tabular}{lrrr}
$\mathrm{H}$ & -0.879350 & 6.073661 & 1.335083 \\
$\mathrm{H}$ & 2.832277 & 4.360483 & 0.171839 \\
$\mathrm{C}$ & -3.240395 & 1.211235 & 4.123199 \\
$\mathrm{H}$ & -1.284407 & 1.194733 & 5.020276 \\
$\mathrm{H}$ & -5.028789 & 1.285964 & 2.930203 \\
$\mathrm{H}$ & -4.584322 & 1.763723 & -4.227372 \\
$\mathrm{C}$ & -3.235419 & -3.307758 & -0.346402 \\
$\mathrm{~N}$ & -4.693786 & -1.363425 & -0.012925 \\
$\mathrm{C}$ & -1.220586 & -3.154815 & -1.583165 \\
$\mathrm{H}$ & -0.769113 & -1.255119 & -2.441830 \\
$\mathrm{H}$ & 1.504303 & 6.311384 & 0.921102 \\
$\mathrm{H}$ & -3.767309 & 1.235083 & 5.068184 \\
$\mathrm{C}$ & -2.081575 & -3.892207 & -0.870211 \\
$\mathrm{H}$ & -3.929161 & -3.919041 & 0.215832 \\
$\mathrm{C}$ & -4.990510 & -1.580462 & 1.404248 \\
$\mathrm{C}$ & -5.818522 & -1.047104 & -0.894439 \\
$\mathrm{H}$ & -0.344660 & -3.621711 & -2.009116 \\
$\mathrm{H}$ & -1.888071 & -4.944758 & -0.707245 \\
$\mathrm{H}$ & -5.665553 & -0.783116 & 1.778040 \\
$\mathrm{H}$ & -5.494838 & -2.560371 & 1.538861 \\
$\mathrm{H}$ & -4.054747 & -1.570852 & 2.003458 \\
$\mathrm{H}$ & -6.753634 & -1.511005 & -0.513289 \\
$\mathrm{H}$ & -5.955683 & 0.053287 & -0.939828 \\
$\mathrm{H}$ & -5.652627 & -1.448777 & -1.914473 \\
$\mathrm{H}$ & 2.086819 & -4.249613 & -2.468530 \\
$\mathrm{~F}$ & 2.511935 & -5.434207 & -0.181082 \\
\hline \hline
\end{tabular}

Table S29: Cartesian coordinates $(\AA)$ of t2hTS $(\mathrm{E}=$ 1487.188208645585 a.u.).

\begin{tabular}{lrrr}
\hline $\mathrm{C}$ & -0.101283 & -2.805698 & 2.894018 \\
$\mathrm{C}$ & -0.009815 & -2.097988 & 1.690360 \\
$\mathrm{C}$ & 0.885441 & -1.033302 & 1.528601 \\
$\mathrm{C}$ & 1.719408 & -0.684748 & 2.609839 \\
$\mathrm{C}$ & 1.613746 & -1.393384 & 3.815849 \\
$\mathrm{C}$ & 0.709898 & -2.446349 & 3.968777 \\
$\mathrm{Pd}$ & 0.901529 & 0.070018 & -0.190703 \\
$\mathrm{C}$ & 2.772177 & 0.383105 & 2.443608 \\
$\mathrm{C}$ & 4.052060 & -0.181659 & 1.777866 \\
$\mathrm{C}$ & 3.803360 & -1.110657 & 0.610812 \\
$\mathrm{C}$ & 3.030431 & -0.670959 & -0.510182 \\
$\mathrm{C}$ & 2.995856 & -1.556682 & -1.601320 \\
$\mathrm{C}$ & 3.583430 & -2.820000 & -1.623408 \\
$\mathrm{C}$ & 4.275050 & -3.200182 & -0.485196 \\
$\mathrm{C}$ & 4.409204 & -2.368420 & 0.621646 \\
$\mathrm{H}$ & -0.675003 & -2.368148 & 0.881265 \\
$\mathrm{H}$ & 4.991367 & -2.709605 & 1.472208 \\
$\mathrm{H}$ & 2.256035 & -1.108374 & 4.648626 \\
$\mathrm{H}$ & 0.640778 & -2.976014 & 4.916694 \\
$\mathrm{H}$ & -0.814998 & -3.622549 & 2.986652 \\
$\mathrm{H}$ & 3.299516 & 0.571395 & -0.796579
\end{tabular}




\begin{tabular}{|c|c|c|c|}
\hline $\mathrm{H}$ & 3.050427 & 0.814480 & 3.413368 \\
\hline $\mathrm{H}$ & 2.400880 & 1.206744 & 1.829852 \\
\hline $\mathrm{H}$ & 4.645774 & -0.716743 & 2.530084 \\
\hline $\mathrm{H}$ & 4.629273 & 0.684934 & 1.426204 \\
\hline $\mathrm{Br}$ & 0.630138 & 1.652233 & -2.394487 \\
\hline $\mathrm{O}$ & 3.882160 & 1.682152 & -1.202664 \\
\hline $\mathrm{C}$ & 3.848864 & 2.723068 & -0.430976 \\
\hline $\mathrm{O}$ & 4.229808 & 2.833907 & 0.733901 \\
\hline $\mathrm{O}$ & 3.316086 & 3.834358 & -1.050464 \\
\hline $\mathrm{H}$ & 2.887751 & 3.477091 & -1.850634 \\
\hline $\mathrm{F}$ & 2.381182 & -1.184515 & -2.738898 \\
\hline $\mathrm{P}$ & -1.229316 & 0.998082 & 0.240358 \\
\hline $\mathrm{C}$ & -2.395582 & 0.945047 & -1.189969 \\
\hline $\mathrm{C}$ & -0.974825 & 2.750679 & 0.690441 \\
\hline $\mathrm{C}$ & -2.175062 & 0.464540 & 1.719649 \\
\hline $\mathrm{C}$ & -2.726841 & -0.276100 & -1.851063 \\
\hline $\mathrm{C}$ & -2.943476 & 2.148711 & -1.689144 \\
\hline $\mathrm{C}$ & -2.037509 & 3.538622 & 1.177546 \\
\hline $\mathrm{C}$ & 0.270277 & 3.269342 & 0.657405 \\
\hline $\mathrm{C}$ & -1.578224 & 0.515117 & 2.929895 \\
\hline $\mathrm{C}$ & -3.545813 & 0.156264 & 1.634259 \\
\hline $\mathrm{C}$ & -3.556858 & -0.225167 & -2.983604 \\
\hline C & -2.091696 & -1.584598 & -1.545632 \\
\hline $\mathrm{C}$ & -3.772437 & 2.162244 & -2.743036 \\
\hline $\mathrm{H}$ & -2.692635 & 3.100278 & -1.254630 \\
\hline $\mathrm{H}$ & -3.040193 & 3.136724 & 1.247172 \\
\hline $\mathrm{C}$ & -1.792769 & 4.859961 & 1.569511 \\
\hline C & 0.504124 & 4.540589 & 1.038856 \\
\hline $\mathrm{H}$ & 1.100458 & 2.659648 & 0.331582 \\
\hline $\mathrm{H}$ & -0.547268 & 0.828451 & 3.016222 \\
\hline $\mathrm{C}$ & -2.280406 & 0.200138 & 4.099401 \\
\hline $\mathrm{C}$ & -4.276377 & -0.130184 & 2.792830 \\
\hline $\mathrm{H}$ & -4.062946 & 0.163817 & 0.686198 \\
\hline $\mathrm{C}$ & -4.098145 & 0.984047 & -3.409217 \\
\hline $\mathrm{H}$ & -3.755454 & -1.116832 & -3.563713 \\
\hline $\mathrm{C}$ & -2.800221 & -2.654252 & -0.926239 \\
\hline $\mathrm{C}$ & -0.852916 & -1.817441 & -2.034399 \\
\hline $\mathrm{H}$ & -4.174287 & 3.102696 & -3.098982 \\
\hline $\mathrm{C}$ & -0.545471 & 5.352385 & 1.497668 \\
\hline $\mathrm{H}$ & -2.603712 & 5.477994 & 1.933037 \\
\hline $\mathrm{H}$ & 1.510552 & 4.937637 & 1.000957 \\
\hline $\mathrm{C}$ & -3.635558 & -0.126205 & 4.031581 \\
\hline $\mathrm{H}$ & -1.778376 & 0.233146 & 5.057655 \\
\hline $\mathrm{H}$ & -5.333680 & -0.351585 & 2.730026 \\
\hline $\mathrm{H}$ & -4.742922 & 1.013343 & -4.278105 \\
\hline $\mathrm{C}$ & -2.176912 & -3.912360 & -0.829491 \\
\hline $\mathrm{N}$ & -4.124959 & -2.488056 & -0.384754 \\
\hline $\mathrm{C}$ & -0.250137 & -3.079271 & -1.927800 \\
\hline $\mathrm{H}$ & -0.320840 & -1.039392 & -2.561662 \\
\hline $\mathrm{H}$ & -0.346308 & 6.371842 & 1.802473 \\
\hline $\mathrm{H}$ & -4.188389 & -0.354622 & 4.933449 \\
\hline
\end{tabular}




\begin{tabular}{lrrr}
$\mathrm{C}$ & -0.892626 & -4.097986 & -1.342498 \\
$\mathrm{H}$ & -2.687727 & -4.748356 & -0.369970 \\
$\mathrm{C}$ & -4.388817 & -2.946758 & 0.980504 \\
$\mathrm{C}$ & -5.269881 & -2.373184 & -1.289174 \\
$\mathrm{H}$ & 0.734453 & -3.240973 & -2.343438 \\
$\mathrm{H}$ & -0.418974 & -5.068929 & -1.272825 \\
$\mathrm{H}$ & -5.273250 & -2.420514 & 1.395266 \\
$\mathrm{H}$ & -4.598001 & -4.037113 & 0.979105 \\
$\mathrm{H}$ & -3.512065 & -2.747226 & 1.633518 \\
$\mathrm{H}$ & -6.057688 & -3.107140 & -1.014224 \\
$\mathrm{H}$ & -5.693277 & -1.349421 & -1.222803 \\
$\mathrm{H}$ & -4.976568 & -2.593058 & -2.335487 \\
$\mathrm{H}$ & 3.511115 & -3.465646 & -2.490874 \\
$\mathrm{~F}$ & 4.860931 & -4.420717 & -0.459772 \\
\hline \hline
\end{tabular}

Table S30: Cartesian coordinates $(\AA)$ of t2iR $(\mathrm{E}=$ 1487.194914133937 a.u.).

\begin{tabular}{lrrr}
\hline \hline $\mathrm{C}$ & -0.675311 & -2.846474 & 2.806833 \\
$\mathrm{C}$ & -0.540096 & -2.122331 & 1.617995 \\
$\mathrm{C}$ & 0.597168 & -1.348806 & 1.358056 \\
$\mathrm{C}$ & 1.621013 & -1.281865 & 2.324732 \\
$\mathrm{C}$ & 1.466739 & -2.009095 & 3.516241 \\
$\mathrm{C}$ & 0.334136 & -2.784110 & 3.765762 \\
$\mathrm{Pd}$ & 0.750860 & -0.275828 & -0.377490 \\
$\mathrm{C}$ & 2.890470 & -0.509097 & 2.070568 \\
$\mathrm{C}$ & 3.916825 & -1.333141 & 1.242106 \\
$\mathrm{C}$ & 3.295027 & -2.030154 & 0.063931 \\
$\mathrm{C}$ & 2.985169 & -1.318025 & -1.123499 \\
$\mathrm{C}$ & 2.517331 & -2.039383 & -2.237176 \\
$\mathrm{C}$ & 2.268805 & -3.407754 & -2.191921 \\
$\mathrm{C}$ & 2.521491 & -4.093172 & -1.004387 \\
$\mathrm{C}$ & 3.023910 & -3.400255 & 0.088674 \\
$\mathrm{H}$ & -1.351844 & -2.146022 & 0.904222 \\
$\mathrm{H}$ & 2.258804 & -1.955814 & 4.261442 \\
$\mathrm{H}$ & 0.240175 & -3.328035 & 4.703236 \\
$\mathrm{H}$ & -1.571881 & -3.438886 & 2.979455 \\
$\mathrm{H}$ & 3.374959 & -0.298235 & -1.221934 \\
$\mathrm{H}$ & 3.369826 & -0.221340 & 3.012337 \\
$\mathrm{H}$ & 2.713905 & 0.428665 & 1.537057 \\
$\mathrm{H}$ & 4.385781 & -2.082060 & 1.887998 \\
$\mathrm{H}$ & 4.663111 & -0.622277 & 0.866088 \\
$\mathrm{Br}$ & 0.773807 & 1.245234 & -2.567079 \\
$\mathrm{O}$ & 4.951777 & 0.953310 & -0.637356 \\
$\mathrm{C}$ & 4.900573 & 1.941389 & 0.154221 \\
$\mathrm{O}$ & 4.502952 & 2.021175 & 1.327377 \\
$\mathrm{O}$ & 5.392892 & 3.146531 & -0.401972 \\
$\mathrm{H}$ & 5.614045 & 2.878131 & -1.308708 \\
$\mathrm{~F}$ & 2.374343 & -1.423271 & -3.419184 \\
$\mathrm{P}$ & -0.894562 & 1.205520 & 0.256603 \\
$\mathrm{C}$ & -2.173716 & 1.495388 & -1.043497
\end{tabular}




\begin{tabular}{|c|c|c|c|}
\hline C & -0.096083 & 2.807572 & 0.624083 \\
\hline $\mathrm{C}$ & -1.781972 & 0.944585 & 1.839406 \\
\hline $\mathrm{C}$ & -2.893928 & 0.425256 & -1.657860 \\
\hline $\mathrm{C}$ & -2.413830 & 2.812319 & -1.498426 \\
\hline $\mathrm{C}$ & -0.824747 & 3.854240 & 1.225696 \\
\hline $\mathrm{C}$ & 1.226065 & 2.966802 & 0.401518 \\
\hline $\mathrm{C}$ & -1.067118 & 0.836716 & 2.980231 \\
\hline $\mathrm{C}$ & -3.186573 & 1.004762 & 1.903686 \\
\hline $\mathrm{C}$ & -3.776265 & 0.724229 & -2.709798 \\
\hline $\mathrm{C}$ & -2.639515 & -1.014845 & -1.391513 \\
\hline $\mathrm{C}$ & -3.299966 & 3.073047 & -2.470292 \\
\hline $\mathrm{H}$ & -1.873755 & 3.650844 & -1.095903 \\
\hline $\mathrm{H}$ & -1.879515 & 3.743390 & 1.442459 \\
\hline $\mathrm{C}$ & -0.178705 & 5.056218 & 1.535844 \\
\hline $\mathrm{C}$ & 1.844832 & 4.126645 & 0.701075 \\
\hline $\mathrm{H}$ & 1.808704 & 2.157700 & -0.017346 \\
\hline $\mathrm{H}$ & 0.013309 & 0.867764 & 2.951393 \\
\hline $\mathrm{C}$ & -1.698565 & 0.715517 & 4.223814 \\
\hline $\mathrm{C}$ & -3.839061 & 0.917053 & 3.138556 \\
\hline $\mathrm{H}$ & -3.781133 & 1.147763 & 1.013524 \\
\hline $\mathrm{C}$ & -3.996944 & 2.042834 & -3.094734 \\
\hline $\mathrm{H}$ & -4.268318 & -0.067047 & -3.260034 \\
\hline $\mathrm{C}$ & -3.575164 & -1.841053 & -0.704141 \\
\hline $\mathrm{C}$ & -1.569541 & -1.592851 & -1.982586 \\
\hline $\mathrm{H}$ & -3.457987 & 4.094251 & -2.794067 \\
\hline $\mathrm{C}$ & 1.130968 & 5.190800 & 1.273982 \\
\hline $\mathrm{H}$ & -0.732094 & 5.870379 & 1.986013 \\
\hline $\mathrm{H}$ & 2.902742 & 4.240111 & 0.505342 \\
\hline $\mathrm{C}$ & -3.091257 & 0.753484 & 4.304381 \\
\hline $\mathrm{H}$ & -1.106348 & 0.615760 & 5.124174 \\
\hline $\mathrm{H}$ & -4.917930 & 0.979648 & 3.191336 \\
\hline $\mathrm{H}$ & -4.684911 & 2.264476 & -3.900433 \\
\hline $\mathrm{C}$ & -3.338365 & -3.226993 & -0.643522 \\
\hline $\mathrm{N}$ & -4.746681 & -1.305070 & -0.059539 \\
\hline $\mathrm{C}$ & -1.351820 & -2.975568 & -1.909671 \\
\hline $\mathrm{H}$ & -0.882779 & -1.001842 & -2.568164 \\
\hline $\mathrm{H}$ & 1.639400 & 6.116545 & 1.511419 \\
\hline $\mathrm{H}$ & -3.585330 & 0.676654 & 5.264153 \\
\hline $\mathrm{C}$ & -2.210105 & -3.771051 & -1.258592 \\
\hline $\mathrm{H}$ & -4.030351 & -3.883586 & -0.132469 \\
\hline $\mathrm{C}$ & -5.012041 & -1.671340 & 1.332874 \\
\hline $\mathrm{C}$ & -5.886181 & -0.871650 & -0.869348 \\
\hline $\mathrm{H}$ & -0.495883 & -3.408633 & -2.406408 \\
\hline $\mathrm{H}$ & -2.034382 & -4.838412 & -1.216355 \\
\hline $\mathrm{H}$ & -5.654245 & -0.903857 & 1.812165 \\
\hline $\mathrm{H}$ & -5.539997 & -2.647289 & 1.372570 \\
\hline $\mathrm{H}$ & -4.061076 & -1.752374 & 1.901998 \\
\hline $\mathrm{H}$ & -6.821352 & -1.356849 & -0.515834 \\
\hline $\mathrm{H}$ & -6.000125 & 0.229427 & -0.789437 \\
\hline $\mathrm{H}$ & -5.755216 & -1.161163 & -1.931583 \\
\hline $\mathrm{H}$ & 2.343831 & -5.160836 & -0.919402 \\
\hline
\end{tabular}




\begin{tabular}{lllr}
$\mathrm{F}$ & 3.285857 & -4.107680 & 1.217778 \\
$\mathrm{H}$ & 1.915550 & -3.917963 & -3.081821 \\
\hline \hline
\end{tabular}

Table S31: Cartesian coordinates $(\AA)$ of t2iTS $(\mathrm{E}=$ 1487.185016279478 a.u.).

\begin{tabular}{lrrr}
\hline \hline $\mathrm{C}$ & -0.087987 & -3.112483 & 2.542733 \\
$\mathrm{C}$ & -0.034707 & -2.280107 & 1.419294 \\
$\mathrm{C}$ & 0.904305 & -1.246595 & 1.313939 \\
$\mathrm{C}$ & 1.820613 & -1.056765 & 2.367419 \\
$\mathrm{C}$ & 1.753151 & -1.890940 & 3.493359 \\
$\mathrm{C}$ & 0.807008 & -2.912426 & 3.592063 \\
$\mathrm{Pd}$ & 0.883427 & 0.025930 & -0.284580 \\
$\mathrm{C}$ & 2.915499 & -0.025264 & 2.250665 \\
$\mathrm{C}$ & 4.132887 & -0.574364 & 1.463806 \\
$\mathrm{C}$ & 3.768030 & -1.363361 & 0.228252 \\
$\mathrm{C}$ & 2.968849 & -0.789336 & -0.806320 \\
$\mathrm{C}$ & 2.806261 & -1.557788 & -1.970172 \\
$\mathrm{C}$ & 3.307174 & -2.847033 & -2.136617 \\
$\mathrm{C}$ & 4.045105 & -3.403048 & -1.096927 \\
$\mathrm{C}$ & 4.269999 & -2.649521 & 0.051754 \\
$\mathrm{H}$ & -0.761800 & -2.429115 & 0.632312 \\
$\mathrm{H}$ & 2.461944 & -1.731638 & 4.305153 \\
$\mathrm{H}$ & 0.770614 & -3.541515 & 4.479021 \\
$\mathrm{H}$ & -0.836621 & -3.901198 & 2.594499 \\
$\mathrm{H}$ & 3.261738 & 0.462641 & -0.973146 \\
$\mathrm{H}$ & 3.261661 & 0.292458 & 3.242098 \\
$\mathrm{H}$ & 2.554022 & 0.871776 & 1.743151 \\
$\mathrm{H}$ & 4.736749 & -1.206687 & 2.120648 \\
$\mathrm{H}$ & 4.730086 & 0.297317 & 1.162941 \\
$\mathrm{Br}$ & 0.567285 & 1.836923 & -2.296909 \\
$\mathrm{O}$ & 3.886065 & 1.586645 & -1.299012 \\
$\mathrm{C}$ & 3.941075 & 2.547884 & -0.430947 \\
$\mathrm{O}$ & 4.402321 & 2.533867 & 0.709660 \\
$\mathrm{O}$ & 3.407520 & 3.729090 & -0.903754 \\
$\mathrm{H}$ & 2.912230 & 3.462110 & -1.700816 \\
$\mathrm{~F}$ & 2.150007 & -1.035440 & -3.026274 \\
$\mathrm{P}$ & -1.163606 & 0.998785 & 0.371389 \\
$\mathrm{C}$ & -2.412150 & 1.150176 & -0.980494 \\
$\mathrm{C}$ & -0.792537 & 2.680905 & 0.980699 \\
$\mathrm{C}$ & -2.048077 & 0.357966 & 1.846013 \\
$\mathrm{C}$ & -2.846608 & 0.021869 & -1.739631 \\
$\mathrm{C}$ & -2.921726 & 2.424219 & -1.320358 \\
$\mathrm{C}$ & -1.782915 & 3.461776 & 1.610684 \\
$\mathrm{C}$ & 0.474072 & 3.141758 & 0.923672 \\
$\mathrm{C}$ & -1.380952 & 0.257802 & 3.015894 \\
$\mathrm{C}$ & -3.435358 & 0.121808 & 1.816071 \\
$\mathrm{C}$ & -3.735705 & 0.230467 & -2.807440 \\
-2.267507 & -1.340493 & -1.608121 \\
$\mathrm{C}$ & -3.807587 & 2.587306 & -2.313660
\end{tabular}




$\begin{array}{lrrr}\mathrm{H} & -2.595073 & 3.312331 & -0.808750 \\ \mathrm{H} & -2.799743 & 3.101705 & 1.700999 \\ \mathrm{C} & -1.447115 & 4.721814 & 2.119635 \\ \mathrm{C} & 0.795897 & 4.353579 & 1.417856 \\ \mathrm{H} & 1.250724 & 2.531431 & 0.486509 \\ \mathrm{H} & -0.332275 & 0.514735 & 3.068842 \\ \mathrm{C} & -2.029795 & -0.143762 & 4.189661 \\ \mathrm{C} & -4.111717 & -0.248845 & 2.983659 \\ \mathrm{H} & -4.005167 & 0.250173 & 0.907719 \\ \mathrm{C} & -4.234078 & 1.502010 & -3.074013 \\ \mathrm{H} & -4.014920 & -0.585206 & -3.461443 \\ \mathrm{C} & -2.997352 & -2.433611 & -1.058169 \\ \mathrm{C} & -1.072569 & -1.580508 & -2.193095 \\ \mathrm{H} & -4.177444 & 3.577964 & -2.546987 \\ \mathrm{C} & -0.181716 & 5.160558 & 2.021349 \\ \mathrm{H} & -2.202422 & 5.335204 & 2.593980 \\ \mathrm{H} & 1.818071 & 4.705154 & 1.358630 \\ \mathrm{C} & -3.401389 & -0.400566 & 4.174258 \\ \mathrm{H} & -1.472531 & -0.231383 & 5.113325 \\ \mathrm{H} & -5.180828 & -0.414640 & 2.965328 \\ \mathrm{H} & -4.924736 & 1.652656 & -3.893684 \\ \mathrm{C} & -2.438493 & -3.723408 & -1.127329 \\ \mathrm{~N} & -4.279134 & -2.261455 & -0.423115 \\ \mathrm{C} & -0.534269 & -2.874170 & -2.252223 \\ \mathrm{H} & -0.529300 & -0.778246 & -2.670324 \\ \mathrm{H} & 0.087875 & 6.131933 & 2.415834 \\ \mathrm{H} & -3.912381 & -0.695093 & 5.081619 \\ \mathrm{C} & -1.197022 & -3.916170 & -1.734417 \\ \mathrm{H} & -2.967297 & -4.577191 & -0.724327 \\ \mathrm{C} & -4.491549 & -2.847590 & 0.901761 \\ \mathrm{C} & -5.464575 & -1.997447 & -1.239866 \\ \mathrm{H} & 0.414937 & -3.038914 & -2.742177 \\ \mathrm{H} & -0.773580 & -4.910772 & -1.792658 \\ \mathrm{H} & -5.320339 & -2.324183 & 1.421689 \\ \mathrm{H} & -4.761565 & -3.919849 & 0.801782 \\ \mathrm{H} & -3.569792 & -2.762327 & 1.516596 \\ & -6.275201 & -2.716208 & -0.992470 \\ \mathrm{H} & -5.826539 & -0.966483 & -1.045303 \\ \mathrm{H} & -5.242563 & -2.122201 & -2.318886 \\ \mathrm{H} & 5.033464 & -3.223582 & -1.165848 \\ \mathrm{H} & & -3.384653 & -3.062256\end{array}$

Table S32: Cartesian coordinates $(\AA)$ of $\mathbf{t 2 j \mathbf { j }}(\mathrm{E}=$ 1487.197488473326 a.u.).

\begin{tabular}{lrrr}
\hline \hline $\mathrm{C}$ & -0.544144 & -2.205583 & 3.333853 \\
$\mathrm{C}$ & -0.430826 & -1.715065 & 2.029066 \\
$\mathrm{C}$ & 0.676524 & -0.960821 & 1.622652 \\
$\mathrm{C}$ & 1.689959 & -0.667564 & 2.557899 \\
$\mathrm{C}$ & 1.554973 & -1.158629 & 3.867511
\end{tabular}




\begin{tabular}{|c|c|c|c|}
\hline $\mathrm{C}$ & 0.455404 & -1.920929 & 4.262012 \\
\hline $\mathrm{Pd}$ & 0.777565 & -0.253348 & -0.295962 \\
\hline $\mathrm{C}$ & 2.933077 & 0.095468 & 2.173692 \\
\hline $\mathrm{C}$ & 4.020378 & -0.835148 & 1.566278 \\
\hline $\mathrm{C}$ & 3.473125 & -1.753284 & 0.509435 \\
\hline $\mathrm{C}$ & 3.000146 & -1.212937 & -0.715687 \\
\hline $\mathrm{C}$ & 2.508919 & -2.071721 & -1.724108 \\
\hline $\mathrm{C}$ & 2.480935 & -3.441761 & -1.510422 \\
\hline $\mathrm{C}$ & 2.925564 & -3.963949 & -0.299813 \\
\hline $\mathrm{C}$ & 3.411369 & -3.135355 & 0.704764 \\
\hline $\mathrm{H}$ & -1.240269 & -1.904779 & 1.337708 \\
\hline $\mathrm{H}$ & 2.336131 & -0.925082 & 4.589614 \\
\hline $\mathrm{H}$ & 0.377221 & -2.278676 & 5.286468 \\
\hline $\mathrm{H}$ & -1.417576 & -2.789937 & 3.616738 \\
\hline $\mathrm{H}$ & 3.269926 & -0.174834 & -0.944365 \\
\hline $\mathrm{H}$ & 3.367087 & 0.599914 & 3.043228 \\
\hline $\mathrm{H}$ & 2.734070 & 0.891219 & 1.451907 \\
\hline $\mathrm{H}$ & 4.483148 & -1.432545 & 2.362714 \\
\hline $\mathrm{H}$ & 4.763193 & -0.174041 & 1.100525 \\
\hline $\mathrm{Br}$ & 0.767190 & 0.760514 & -2.789371 \\
\hline $\mathrm{O}$ & 4.876026 & 1.204058 & -0.616709 \\
\hline $\mathrm{C}$ & 4.838896 & 2.312371 & -0.002850 \\
\hline $\mathrm{O}$ & 4.398981 & 2.604104 & 1.119982 \\
\hline $\mathrm{O}$ & 5.406534 & 3.387218 & -0.729969 \\
\hline $\mathrm{H}$ & 5.656954 & 2.959335 & -1.565027 \\
\hline $\mathrm{P}$ & -0.945281 & 1.251366 & 0.028732 \\
\hline $\mathrm{C}$ & -2.212705 & 1.221380 & -1.313337 \\
\hline $\mathrm{C}$ & -0.211633 & 2.924095 & 0.060021 \\
\hline $\mathrm{C}$ & -1.841661 & 1.282768 & 1.626383 \\
\hline $\mathrm{C}$ & -2.886180 & 0.021414 & -1.695840 \\
\hline $\mathrm{C}$ & -2.491023 & 2.405484 & -2.033763 \\
\hline $\mathrm{C}$ & -0.982659 & 4.042536 & 0.437484 \\
\hline $\mathrm{C}$ & 1.103420 & 3.085294 & -0.200010 \\
\hline $\mathrm{C}$ & -1.136062 & 1.419453 & 2.769739 \\
\hline $\mathrm{C}$ & -3.247679 & 1.322267 & 1.669165 \\
\hline $\mathrm{C}$ & -3.760836 & 0.061329 & -2.794720 \\
\hline $\mathrm{C}$ & -2.588372 & -1.319437 & -1.127361 \\
\hline $\mathrm{C}$ & -3.370072 & 2.425083 & -3.046102 \\
\hline $\mathrm{H}$ & -1.987443 & 3.329077 & -1.808779 \\
\hline $\mathrm{H}$ & -2.033103 & 3.937734 & 0.676750 \\
\hline $\mathrm{C}$ & -0.384707 & 5.306616 & 0.491435 \\
\hline $\mathrm{C}$ & 1.675346 & 4.305168 & -0.148789 \\
\hline $\mathrm{H}$ & 1.717786 & 2.230542 & -0.449343 \\
\hline $\mathrm{H}$ & -0.056433 & 1.470009 & 2.741425 \\
\hline $\mathrm{C}$ & -1.777364 & 1.532955 & 4.009035 \\
\hline $\mathrm{C}$ & -3.910608 & 1.467319 & 2.892999 \\
\hline $\mathrm{H}$ & -3.835709 & 1.272593 & 0.764532 \\
\hline $\mathrm{C}$ & -4.020721 & 1.261991 & -3.448091 \\
\hline $\mathrm{H}$ & -4.217314 & -0.844909 & -3.170727 \\
\hline $\mathrm{C}$ & -3.504061 & -2.012067 & -0.283304 \\
\hline $\mathrm{C}$ & -1.488262 & -1.969403 & -1.569593 \\
\hline
\end{tabular}




\begin{tabular}{rrrr}
$\mathrm{H}$ & -3.558770 & 3.350144 & -3.576515 \\
$\mathrm{C}$ & 0.919244 & 5.435452 & 0.199463 \\
$\mathrm{H}$ & -0.970547 & 6.173630 & 0.768604 \\
$\mathrm{H}$ & 2.727875 & 4.417453 & -0.372150 \\
$\mathrm{C}$ & -3.171304 & 1.555327 & 4.072385 \\
$\mathrm{H}$ & -1.192292 & 1.627590 & 4.914629 \\
$\mathrm{H}$ & -4.991043 & 1.515122 & 2.926007 \\
$\mathrm{H}$ & -4.703494 & 1.285536 & -4.287630 \\
$\mathrm{C}$ & -3.219108 & -3.342218 & 0.077428 \\
$\mathrm{~N}$ & -4.702283 & -1.393386 & 0.223930 \\
$\mathrm{C}$ & -1.221987 & -3.293922 & -1.195773 \\
$\mathrm{H}$ & -0.811378 & -1.491098 & -2.260361 \\
$\mathrm{H}$ & 1.390435 & 6.409258 & 0.239142 \\
$\mathrm{H}$ & -3.673305 & 1.659952 & 5.025384 \\
$\mathrm{C}$ & -2.062562 & -3.962749 & -0.396184 \\
$\mathrm{H}$ & -3.894574 & -3.898802 & 0.713915 \\
$\mathrm{C}$ & -4.979887 & -1.470332 & 1.659397 \\
$\mathrm{C}$ & -5.839933 & -1.171999 & -0.669810 \\
$\mathrm{H}$ & -0.340138 & -3.789237 & -1.573883 \\
$\mathrm{H}$ & -1.848445 & -4.987559 & -0.120694 \\
$\mathrm{H}$ & -5.650472 & -0.639763 & 1.962086 \\
$\mathrm{H}$ & -5.481490 & -2.432213 & 1.895918 \\
$\mathrm{H}$ & -4.036283 & -1.402718 & 2.242446 \\
$\mathrm{H}$ & -6.768378 & -1.595434 & -0.229803 \\
$\mathrm{H}$ & -5.981337 & -0.082446 & -0.826703 \\
$\mathrm{H}$ & -5.686530 & -1.677210 & -1.644739 \\
$\mathrm{H}$ & 2.189804 & -1.666505 & -2.678040 \\
$\mathrm{~F}$ & 2.044651 & -4.289255 & -2.468800 \\
$\mathrm{~F}$ & 2.882249 & -5.298677 & -0.111922 \\
$\mathrm{H}$ & 3.746297 & -3.587124 & 1.633602 \\
\hline \hline & & &
\end{tabular}

Table S33: Cartesian coordinates $(\AA)$ of t2jTS $(\mathrm{E}=$ 1487.184073643119 a.u.).

\begin{tabular}{lrrr}
\hline \hline $\mathrm{C}$ & -0.177310 & -2.439949 & 3.204262 \\
$\mathrm{C}$ & -0.067452 & -1.829356 & 1.950485 \\
$\mathrm{C}$ & 0.833037 & -0.782727 & 1.711614 \\
$\mathrm{C}$ & 1.651647 & -0.351046 & 2.773826 \\
$\mathrm{C}$ & 1.528145 & -0.961719 & 4.031254 \\
$\mathrm{C}$ & 0.621628 & -1.998512 & 4.257220 \\
$\mathrm{Pd}$ & 0.870716 & 0.138271 & -0.116539 \\
$\mathrm{C}$ & 2.707144 & 0.700740 & 2.546365 \\
$\mathrm{C}$ & 4.003928 & 0.090470 & 1.964864 \\
$\mathrm{C}$ & 3.805449 & -0.854431 & 0.798930 \\
$\mathrm{C}$ & 3.016384 & -0.470942 & -0.324460 \\
$\mathrm{C}$ & 2.994279 & -1.336188 & -1.446051 \\
$\mathrm{C}$ & 3.676680 & -2.541966 & -1.437368 \\
$\mathrm{C}$ & 4.412479 & -2.909904 & -0.315819 \\
$\mathrm{C}$ & 4.490303 & -2.072539 & 0.790801 \\
$\mathrm{H}$ & -0.727208 & -2.158542 & 1.159821 \\
$\mathrm{H}$ & 2.160157 & -0.610986 & 4.846706
\end{tabular}




\begin{tabular}{|c|c|c|c|}
\hline $\mathrm{H}$ & 0.539933 & -2.450825 & 5.243498 \\
\hline $\mathrm{H}$ & -0.895535 & -3.244810 & 3.351289 \\
\hline $\mathrm{H}$ & 3.224968 & 0.811722 & -0.615950 \\
\hline $\mathrm{H}$ & 2.960486 & 1.210334 & 3.484684 \\
\hline $\mathrm{H}$ & 2.352761 & 1.471387 & 1.858904 \\
\hline $\mathrm{H}$ & 4.550290 & -0.435813 & 2.758964 \\
\hline $\mathrm{H}$ & 4.618029 & 0.937299 & 1.629337 \\
\hline $\mathrm{Br}$ & 0.690249 & 1.315244 & -2.550932 \\
\hline $\mathrm{O}$ & 3.768069 & 1.895306 & -1.081188 \\
\hline $\mathrm{C}$ & 3.740500 & 2.978265 & -0.369241 \\
\hline $\mathrm{O}$ & 3.936290 & 3.127341 & 0.833448 \\
\hline $\mathrm{O}$ & 3.457378 & 4.095911 & -1.131444 \\
\hline $\mathrm{H}$ & 3.193056 & 3.729421 & -1.993371 \\
\hline $\mathrm{P}$ & -1.318887 & 0.993213 & 0.088879 \\
\hline $\mathrm{C}$ & -2.404227 & 0.706749 & -1.377850 \\
\hline $\mathrm{C}$ & -1.150828 & 2.798785 & 0.310032 \\
\hline $\mathrm{C}$ & -2.313124 & 0.614924 & 1.581803 \\
\hline $\mathrm{C}$ & -2.648487 & -0.601611 & -1.894385 \\
\hline $\mathrm{C}$ & -2.971664 & 1.811328 & -2.053232 \\
\hline $\mathrm{C}$ & -2.261357 & 3.598387 & 0.648289 \\
\hline $\mathrm{C}$ & 0.074472 & 3.360816 & 0.249415 \\
\hline $\mathrm{C}$ & -1.766733 & 0.819148 & 2.799612 \\
\hline $\mathrm{C}$ & -3.671822 & 0.262664 & 1.479279 \\
\hline $\mathrm{C}$ & -3.414946 & -0.732036 & -3.064614 \\
\hline $\mathrm{C}$ & -1.984711 & -1.831311 & -1.388992 \\
\hline $\mathrm{C}$ & -3.739621 & 1.653922 & -3.141196 \\
\hline $\mathrm{H}$ & -2.785781 & 2.820503 & -1.730600 \\
\hline $\mathrm{H}$ & -3.250313 & 3.167102 & 0.735460 \\
\hline $\mathrm{C}$ & -2.082466 & 4.969561 & 0.865840 \\
\hline $\mathrm{C}$ & 0.244429 & 4.680616 & 0.463233 \\
\hline $\mathrm{H}$ & 0.939953 & 2.748722 & 0.037819 \\
\hline $\mathrm{H}$ & -0.744160 & 1.160005 & 2.888269 \\
\hline $\mathrm{C}$ & -2.510665 & 0.625231 & 3.969721 \\
\hline C & -4.443737 & 0.094157 & 2.634117 \\
\hline $\mathrm{H}$ & -4.147497 & 0.144296 & 0.516670 \\
\hline $\mathrm{C}$ & -3.979229 & 0.387723 & -3.667920 \\
\hline $\mathrm{H}$ & -3.546378 & -1.698153 & -3.534232 \\
\hline $\mathrm{C}$ & -2.686813 & -2.836925 & -0.664581 \\
\hline $\mathrm{C}$ & -0.713026 & -2.073879 & -1.778855 \\
\hline $\mathrm{H}$ & -4.158828 & 2.522486 & -3.633637 \\
\hline $\mathrm{C}$ & -0.852702 & 5.500805 & 0.771295 \\
\hline $\mathrm{H}$ & -2.930098 & 5.595586 & 1.113528 \\
\hline $\mathrm{H}$ & 1.235525 & 5.112198 & 0.406946 \\
\hline $\mathrm{C}$ & -3.855216 & 0.259659 & 3.887881 \\
\hline $\mathrm{H}$ & -2.049352 & 0.780602 & 4.936375 \\
\hline $\mathrm{H}$ & -5.492176 & -0.162110 & 2.556537 \\
\hline $\mathrm{H}$ & -4.575111 & 0.277776 & -4.564802 \\
\hline C & -2.023081 & -4.040653 & -0.361957 \\
\hline $\mathrm{N}$ & -4.043973 & -2.655249 & -0.216567 \\
\hline $\mathrm{C}$ & -0.068555 & -3.278314 & -1.463790 \\
\hline $\mathrm{H}$ & -0.181184 & -1.355013 & -2.383047 \\
\hline
\end{tabular}




\begin{tabular}{lrrr}
$\mathrm{H}$ & -0.704554 & 6.559512 & 0.941775 \\
$\mathrm{H}$ & -4.440114 & 0.123496 & 4.788167 \\
$\mathrm{C}$ & -0.704342 & -4.235355 & -0.775580 \\
$\mathrm{H}$ & -2.527265 & -4.827473 & 0.183712 \\
$\mathrm{C}$ & -4.365633 & -2.931948 & 1.184703 \\
$\mathrm{C}$ & -5.141625 & -2.708945 & -1.183031 \\
$\mathrm{H}$ & 0.945373 & -3.446362 & -1.798544 \\
$\mathrm{H}$ & -0.198016 & -5.163893 & -0.544628 \\
$\mathrm{H}$ & -5.284820 & -2.384868 & 1.479820 \\
$\mathrm{H}$ & -4.543427 & -4.018984 & 1.323939 \\
$\mathrm{H}$ & -3.530186 & -2.614442 & 1.845123 \\
$\mathrm{H}$ & -5.914589 & -3.435835 & -0.852807 \\
$\mathrm{H}$ & -5.606405 & -1.705020 & -1.271982 \\
$\mathrm{H}$ & -4.783846 & -3.047942 & -2.176137 \\
$\mathrm{H}$ & 2.453392 & -1.044441 & -2.341975 \\
$\mathrm{~F}$ & 3.651613 & -3.377581 & -2.503905 \\
$\mathrm{~F}$ & 5.073072 & -4.088601 & -0.320053 \\
$\mathrm{H}$ & 5.099527 & -2.387422 & 1.634131 \\
\hline \hline
\end{tabular}

Table S34: Cartesian coordinates $(\AA)$ of $\mathbf{t 2 k R}(\mathrm{E}=$ 1487.201489110518 a.u.).

\begin{tabular}{lrrr}
\hline \hline $\mathrm{C}$ & -0.471315 & -2.577506 & 3.046095 \\
$\mathrm{C}$ & -0.404264 & -1.945407 & 1.800714 \\
$\mathrm{C}$ & 0.700406 & -1.168792 & 1.430556 \\
$\mathrm{C}$ & 1.758221 & -0.993443 & 2.346518 \\
$\mathrm{C}$ & 1.669876 & -1.629783 & 3.596509 \\
$\mathrm{C}$ & 0.574371 & -2.415426 & 3.952755 \\
$\mathrm{Pd}$ & 0.729868 & -0.273252 & -0.409894 \\
$\mathrm{C}$ & 2.993792 & -0.195347 & 2.008637 \\
$\mathrm{C}$ & 4.068454 & -1.053516 & 1.282396 \\
$\mathrm{C}$ & 3.475245 & -1.857611 & 0.160215 \\
$\mathrm{C}$ & 2.970258 & -1.211519 & -0.995594 \\
$\mathrm{C}$ & 2.384059 & -1.949121 & -2.044893 \\
$\mathrm{C}$ & 2.321462 & -3.334954 & -1.934606 \\
$\mathrm{C}$ & 2.798143 & -4.003454 & -0.817684 \\
$\mathrm{C}$ & 3.354842 & -3.243376 & 0.209501 \\
$\mathrm{H}$ & -1.246812 & -2.041534 & 1.130342 \\
$\mathrm{H}$ & 2.486761 & -1.492093 & 4.303169 \\
$\mathrm{H}$ & 0.535087 & -2.886173 & 4.932693 \\
$\mathrm{H}$ & -1.344105 & -3.175179 & 3.301782 \\
$\mathrm{H}$ & 3.242209 & -0.156740 & -1.128408 \\
$\mathrm{H}$ & 3.447036 & 0.219647 & 2.915001 \\
$\mathrm{H}$ & 2.776252 & 0.668360 & 1.375590 \\
$\mathrm{H}$ & 4.556809 & -1.723770 & 1.996695 \\
$\mathrm{H}$ & 4.793363 & -0.348518 & 0.858446 \\
$\mathrm{Br}$ & 0.625080 & 0.985624 & -2.785629 \\
$\mathrm{O}$ & 4.817371 & 1.195103 & -0.771633 \\
$\mathrm{C}$ & 4.864768 & 2.212672 & -0.018313 \\
$\mathrm{O}$ & 4.472739 & 2.380260 & 1.146955 \\
$\mathrm{O}$ & 5.480939 & 3.338816 & -0.618516
\end{tabular}




\begin{tabular}{|c|c|c|c|}
\hline $\mathrm{H}$ & 5.684055 & 3.008874 & -1.509084 \\
\hline $\mathrm{P}$ & -0.925682 & 1.228390 & 0.155505 \\
\hline $\mathrm{C}$ & -2.257518 & 1.365383 & -1.115947 \\
\hline $\mathrm{C}$ & -0.146709 & 2.872805 & 0.317894 \\
\hline $\mathrm{C}$ & -1.741769 & 1.112257 & 1.792139 \\
\hline $\mathrm{C}$ & -2.979996 & 0.227100 & -1.589059 \\
\hline $\mathrm{C}$ & -2.538176 & 2.624731 & -1.694278 \\
\hline $\mathrm{C}$ & -0.865825 & 3.961350 & 0.852613 \\
\hline $\mathrm{C}$ & 1.154907 & 3.034016 & -0.002435 \\
\hline $\mathrm{C}$ & -0.977924 & 1.121684 & 2.905834 \\
\hline $\mathrm{C}$ & -3.142909 & 1.165070 & 1.910586 \\
\hline $\mathrm{C}$ & -3.901245 & 0.401934 & -2.635518 \\
\hline $\mathrm{C}$ & -2.698735 & -1.172727 & -1.174743 \\
\hline $\mathrm{C}$ & -3.461173 & 2.769944 & -2.655981 \\
\hline $\mathrm{H}$ & -2.001988 & 3.508997 & -1.398151 \\
\hline $\mathrm{H}$ & -1.903494 & 3.853473 & 1.141112 \\
\hline $\mathrm{C}$ & -0.233391 & 5.201018 & 0.999415 \\
\hline $\mathrm{C}$ & 1.759709 & 4.230862 & 0.137688 \\
\hline $\mathrm{H}$ & 1.731732 & 2.197686 & -0.373958 \\
\hline $\mathrm{H}$ & 0.099749 & 1.160430 & 2.827414 \\
\hline $\mathrm{C}$ & -1.554676 & 1.115927 & 4.181434 \\
\hline $\mathrm{C}$ & -3.741561 & 1.191753 & 3.175305 \\
\hline $\mathrm{H}$ & -3.775732 & 1.215642 & 1.036787 \\
\hline $\mathrm{C}$ & -4.158504 & 1.669999 & -3.146872 \\
\hline $\mathrm{H}$ & -4.397419 & -0.449185 & -3.082936 \\
\hline $\mathrm{C}$ & -3.604626 & -1.928236 & -0.375324 \\
\hline $\mathrm{C}$ & -1.633387 & -1.799718 & -1.722707 \\
\hline $\mathrm{H}$ & -3.649796 & 3.749744 & -3.076797 \\
\hline $\mathrm{C}$ & 1.054310 & 5.333738 & 0.644080 \\
\hline $\mathrm{H}$ & -0.779844 & 6.046177 & 1.397985 \\
\hline $\mathrm{H}$ & 2.799743 & 4.345377 & -0.136989 \\
\hline $\mathrm{C}$ & -2.943107 & 1.148996 & 4.318088 \\
\hline $\mathrm{H}$ & -0.923719 & 1.109676 & 5.060775 \\
\hline $\mathrm{H}$ & -4.817929 & 1.249422 & 3.268584 \\
\hline $\mathrm{H}$ & -4.877141 & 1.796608 & -3.946348 \\
\hline $\mathrm{C}$ & -3.344248 & -3.294643 & -0.163358 \\
\hline $\mathrm{N}$ & -4.772538 & -1.340024 & 0.228817 \\
\hline $\mathrm{C}$ & -1.391608 & -3.162231 & -1.498181 \\
\hline $\mathrm{H}$ & -0.967698 & -1.269027 & -2.384944 \\
\hline $\mathrm{H}$ & 1.551764 & 6.288903 & 0.754001 \\
\hline $\mathrm{H}$ & -3.395230 & 1.161254 & 5.301257 \\
\hline $\mathrm{C}$ & -2.222321 & -3.890248 & -0.740913 \\
\hline $\mathrm{H}$ & -4.013850 & -3.897531 & 0.436171 \\
\hline $\mathrm{C}$ & -4.999221 & -1.540806 & 1.661003 \\
\hline $\mathrm{C}$ & -5.939755 & -1.031972 & -0.598754 \\
\hline $\mathrm{H}$ & -0.536920 & -3.636323 & -1.957244 \\
\hline $\mathrm{H}$ & -2.027482 & -4.942973 & -0.580609 \\
\hline $\mathrm{H}$ & -5.627362 & -0.719852 & 2.064313 \\
\hline $\mathrm{H}$ & -5.525897 & -2.503882 & 1.828341 \\
\hline $\mathrm{H}$ & -4.033153 & -1.559716 & 2.209742 \\
\hline $\mathrm{H}$ & -6.854756 & -1.491936 & -0.167300 \\
\hline
\end{tabular}




\begin{tabular}{lrrr}
$\mathrm{H}$ & -6.079517 & 0.067925 & -0.647710 \\
$\mathrm{H}$ & -5.824654 & -1.443579 & -1.621909 \\
$\mathrm{H}$ & 2.737109 & -5.083014 & -0.738007 \\
$\mathrm{~F}$ & 3.810012 & -3.917376 & 1.294393 \\
$\mathrm{H}$ & 2.035539 & -1.449389 & -2.940909 \\
$\mathrm{~F}$ & 1.801880 & -4.064022 & -2.951546 \\
\hline \hline
\end{tabular}

Table S35: Cartesian coordinates $(\AA)$ of t2kTS $(\mathrm{E}=$ 1487.188470670135 a.u.).

\begin{tabular}{lrrr}
\hline $\mathrm{C}$ & -0.126553 & -2.710669 & 2.955174 \\
$\mathrm{C}$ & -0.056606 & -2.005834 & 1.748898 \\
$\mathrm{C}$ & 0.883239 & -0.987942 & 1.540331 \\
$\mathrm{C}$ & 1.784734 & -0.683921 & 2.578933 \\
$\mathrm{C}$ & 1.700013 & -1.389186 & 3.789481 \\
$\mathrm{C}$ & 0.753493 & -2.395119 & 3.988448 \\
$\mathrm{Pd}$ & 0.865236 & 0.076675 & -0.206785 \\
$\mathrm{C}$ & 2.884073 & 0.327183 & 2.372661 \\
$\mathrm{C}$ & 4.115567 & -0.304837 & 1.680235 \\
$\mathrm{C}$ & 3.795362 & -1.136744 & 0.458316 \\
$\mathrm{C}$ & 2.975508 & -0.636527 & -0.592396 \\
$\mathrm{C}$ & 2.815862 & -1.404937 & -1.768542 \\
$\mathrm{C}$ & 3.417146 & -2.651771 & -1.872342 \\
$\mathrm{C}$ & 4.198661 & -3.172333 & -0.851082 \\
$\mathrm{C}$ & 4.375574 & -2.390541 & 0.288277 \\
$\mathrm{H}$ & -0.776687 & -2.237795 & 0.976335 \\
$\mathrm{H}$ & 2.397130 & -1.139245 & 4.588577 \\
$\mathrm{H}$ & 0.704827 & -2.922046 & 4.939164 \\
$\mathrm{H}$ & -0.876760 & -3.489325 & 3.082202 \\
$\mathrm{H}$ & 3.213481 & 0.650963 & -0.785194 \\
$\mathrm{H}$ & 3.212187 & 0.746421 & 3.332240 \\
$\mathrm{H}$ & 2.539987 & 1.168547 & 1.767734 \\
$\mathrm{H}$ & 4.664271 & -0.922619 & 2.397829 \\
$\mathrm{H}$ & 4.762437 & 0.529006 & 1.376375 \\
$\mathrm{Br}$ & 0.609292 & 1.466659 & -2.519011 \\
$\mathrm{O}$ & 3.796984 & 1.744196 & -1.199166 \\
$\mathrm{C}$ & 3.846692 & 2.774907 & -0.414077 \\
$\mathrm{O}$ & 4.135640 & 2.835030 & 0.777445 \\
$\mathrm{O}$ & 3.529935 & 3.947974 & -1.073273 \\
$\mathrm{H}$ & 3.183502 & 3.645903 & -1.931388 \\
$\mathrm{P}$ & -1.252430 & 1.021878 & 0.208604 \\
$\mathrm{C}$ & -2.435172 & 0.921181 & -1.206392 \\
$\mathrm{C}$ & -0.971336 & 2.790134 & 0.570245 \\
$\mathrm{C}$ & -2.178649 & 0.566763 & 1.723702 \\
$\mathrm{C}$ & -2.785672 & -0.322784 & -1.812942 \\
$\mathrm{C}$ & -2.972711 & 2.108936 & -1.752919 \\
$\mathrm{C}$ & -2.014592 & 3.612458 & 1.042024 \\
$\mathrm{C}$ & 0.277860 & 3.292164 & 0.482581 \\
$\mathrm{C}$ & -1.553453 & 0.639457 & 2.918401 \\
$\mathrm{C}$ & -3.558585 & 0.294308 & 1.676599 \\
$\mathrm{C}$ & -3.621816 & -0.310227 & -2.941964 \\
& & &
\end{tabular}




\begin{tabular}{|c|c|c|c|}
\hline C & -2.169544 & -1.625711 & -1.451332 \\
\hline $\mathrm{C}$ & -3.807939 & 2.087588 & -2.801771 \\
\hline $\mathrm{H}$ & -2.709140 & 3.075168 & -1.360408 \\
\hline $\mathrm{H}$ & -3.019691 & 3.226132 & 1.152308 \\
\hline C & -1.747207 & 4.948218 & 1.363989 \\
\hline $\mathrm{C}$ & 0.533413 & 4.577574 & 0.797255 \\
\hline $\mathrm{H}$ & 1.093628 & 2.657360 & 0.167024 \\
\hline $\mathrm{H}$ & -0.510495 & 0.920445 & 2.971870 \\
\hline $\mathrm{C}$ & -2.239066 & 0.384843 & 4.112251 \\
\hline C & -4.271678 & 0.068203 & 2.859104 \\
\hline $\mathrm{H}$ & -4.094462 & 0.283309 & 0.738760 \\
\hline $\mathrm{C}$ & -4.151077 & 0.886250 & -3.415823 \\
\hline $\mathrm{H}$ & -3.835971 & -1.223792 & -3.481145 \\
\hline $\mathrm{C}$ & -2.888715 & -2.649675 & -0.770404 \\
\hline $\mathrm{C}$ & -0.938663 & -1.903392 & -1.936876 \\
\hline $\mathrm{H}$ & -4.201313 & 3.016786 & -3.194820 \\
\hline $\mathrm{C}$ & -0.496855 & 5.422287 & 1.240306 \\
\hline $\mathrm{H}$ & -2.543126 & 5.592361 & 1.715171 \\
\hline $\mathrm{H}$ & 1.542358 & 4.961415 & 0.717862 \\
\hline $\mathrm{C}$ & -3.604342 & 0.096334 & 4.083616 \\
\hline $\mathrm{H}$ & -1.715539 & 0.434358 & 5.058208 \\
\hline $\mathrm{H}$ & -5.335650 & -0.126028 & 2.825566 \\
\hline $\mathrm{H}$ & -4.801015 & 0.886351 & -4.281353 \\
\hline $\mathrm{C}$ & -2.281799 & -3.909231 & -0.608398 \\
\hline $\mathrm{N}$ & -4.206515 & -2.434082 & -0.229241 \\
\hline $\mathrm{C}$ & -0.351170 & -3.164199 & -1.762609 \\
\hline $\mathrm{H}$ & -0.399393 & -1.165429 & -2.510921 \\
\hline $\mathrm{H}$ & -0.280159 & 6.452865 & 1.490954 \\
\hline $\mathrm{H}$ & -4.143727 & -0.085573 & 5.004015 \\
\hline $\mathrm{C}$ & -1.002899 & -4.140094 & -1.117245 \\
\hline $\mathrm{H}$ & -2.800503 & -4.711201 & -0.099661 \\
\hline C & -4.468545 & -2.816625 & 1.159529 \\
\hline $\mathrm{C}$ & -5.355978 & -2.341307 & -1.130358 \\
\hline $\mathrm{H}$ & 0.629638 & -3.358846 & -2.172493 \\
\hline $\mathrm{H}$ & -0.540978 & -5.111508 & -0.995276 \\
\hline $\mathrm{H}$ & -5.335140 & -2.247171 & 1.554500 \\
\hline $\mathrm{H}$ & -4.705180 & -3.899960 & 1.214715 \\
\hline $\mathrm{H}$ & -3.580764 & -2.607380 & 1.794352 \\
\hline $\mathrm{H}$ & -6.153943 & -3.048061 & -0.816269 \\
\hline $\mathrm{H}$ & -5.761424 & -1.308381 & -1.108658 \\
\hline $\mathrm{H}$ & -5.074166 & -2.614177 & -2.167312 \\
\hline $\mathrm{H}$ & 4.673789 & -4.142998 & -0.938358 \\
\hline $\mathrm{F}$ & 5.176783 & -2.908193 & 1.261251 \\
\hline $\mathrm{H}$ & 2.244386 & -1.009852 & -2.602502 \\
\hline $\mathrm{F}$ & 3.256509 & -3.393405 & -2.999498 \\
\hline
\end{tabular}

Table S36: Cartesian coordinates $(\AA)$ of t2lR (E= 1487.198417086782 a.u.).

\begin{tabular}{llll}
\hline \hline $\mathrm{C}$ & -0.242045 & -2.836875 & 2.758392 \\
$\mathrm{C}$ & -0.230412 & -2.104756 & 1.566980
\end{tabular}




\begin{tabular}{|c|c|c|c|}
\hline C & 0.800459 & -1.205841 & 1.268720 \\
\hline $\mathrm{C}$ & 1.844548 & -1.020549 & 2.198759 \\
\hline $\mathrm{C}$ & 1.812169 & -1.758186 & 3.394315 \\
\hline $\mathrm{C}$ & 0.785820 & -2.657584 & 3.682044 \\
\hline $\mathrm{Pd}$ & 0.730918 & -0.152747 & -0.486162 \\
\hline $\mathrm{C}$ & 3.015148 & -0.108963 & 1.919546 \\
\hline $\mathrm{C}$ & 4.134099 & -0.825669 & 1.108717 \\
\hline $\mathrm{C}$ & 3.564941 & -1.586959 & -0.053449 \\
\hline $\mathrm{C}$ & 2.971298 & -0.891122 & -1.142320 \\
\hline $\mathrm{C}$ & 2.405327 & -1.604307 & -2.218915 \\
\hline $\mathrm{C}$ & 2.412786 & -3.004230 & -2.232039 \\
\hline $\mathrm{C}$ & 2.988107 & -3.669967 & -1.163932 \\
\hline $\mathrm{C}$ & 3.548583 & -2.973599 & -0.089012 \\
\hline $\mathrm{H}$ & -1.056002 & -2.225213 & 0.879336 \\
\hline $\mathrm{H}$ & 2.617275 & -1.612128 & 4.112831 \\
\hline $\mathrm{H}$ & 0.788109 & -3.206804 & 4.621060 \\
\hline $\mathrm{H}$ & -1.058303 & -3.527900 & 2.959855 \\
\hline $\mathrm{H}$ & 3.193567 & 0.180741 & -1.213943 \\
\hline $\mathrm{H}$ & 3.456026 & 0.256831 & 2.852733 \\
\hline $\mathrm{H}$ & 2.726751 & 0.786664 & 1.364331 \\
\hline $\mathrm{H}$ & 4.691607 & -1.505788 & 1.760042 \\
\hline $\mathrm{H}$ & 4.788661 & -0.036899 & 0.717141 \\
\hline $\mathrm{Br}$ & 0.496427 & 1.296065 & -2.739772 \\
\hline $\mathrm{O}$ & 4.724086 & 1.587049 & -0.799562 \\
\hline $\mathrm{C}$ & 4.673006 & 2.558724 & 0.012069 \\
\hline $\mathrm{O}$ & 4.318118 & 2.606014 & 1.200262 \\
\hline $\mathrm{O}$ & 5.104958 & 3.788966 & -0.541456 \\
\hline $\mathrm{H}$ & 5.303691 & 3.541067 & -1.459183 \\
\hline $\mathrm{P}$ & -1.030997 & 1.167205 & 0.228535 \\
\hline $\mathrm{C}$ & -2.390297 & 1.322292 & -1.011485 \\
\hline $\mathrm{C}$ & -0.369981 & 2.843110 & 0.534493 \\
\hline $\mathrm{C}$ & -1.814607 & 0.848110 & 1.854228 \\
\hline C & -3.036947 & 0.182543 & -1.579790 \\
\hline $\mathrm{C}$ & -2.774299 & 2.605533 & -1.464195 \\
\hline $\mathrm{C}$ & -1.156628 & 3.822750 & 1.174694 \\
\hline $\mathrm{C}$ & 0.912304 & 3.126651 & 0.221135 \\
\hline $\mathrm{C}$ & -1.038100 & 0.805906 & 2.958215 \\
\hline $\mathrm{C}$ & -3.214789 & 0.801609 & 1.988390 \\
\hline C & -3.993134 & 0.384483 & -2.589505 \\
\hline $\mathrm{C}$ & -2.636334 & -1.223921 & -1.314429 \\
\hline $\mathrm{C}$ & -3.726769 & 2.770328 & -2.393488 \\
\hline $\mathrm{H}$ & -2.297561 & 3.495893 & -1.094014 \\
\hline $\mathrm{H}$ & -2.179848 & 3.614517 & 1.459766 \\
\hline $\mathrm{C}$ & -0.611263 & 5.085269 & 1.433505 \\
\hline $\mathrm{C}$ & 1.433184 & 4.345160 & 0.470131 \\
\hline $\mathrm{H}$ & 1.541566 & 2.372292 & -0.231850 \\
\hline $\mathrm{H}$ & 0.033217 & 0.923478 & 2.875806 \\
\hline $\mathrm{C}$ & -1.596018 & 0.647237 & 4.232411 \\
\hline $\mathrm{C}$ & -3.796821 & 0.676198 & 3.254721 \\
\hline $\mathrm{H}$ & -3.861902 & 0.891886 & 1.128454 \\
\hline $\mathrm{C}$ & -4.353959 & 1.671883 & -2.974312 \\
\hline
\end{tabular}




\begin{tabular}{lrrr}
$\mathrm{H}$ & -4.437106 & -0.456622 & -3.106095 \\
$\mathrm{C}$ & -3.441011 & -2.117209 & -0.549222 \\
$\mathrm{C}$ & -1.565422 & -1.715146 & -1.977813 \\
$\mathrm{H}$ & -3.995474 & 3.768230 & -2.716962 \\
$\mathrm{C}$ & 0.658919 & 5.342327 & 1.083656 \\
$\mathrm{H}$ & -1.210318 & 5.848002 & 1.914231 \\
$\mathrm{H}$ & 2.458816 & 4.558942 & 0.201170 \\
$\mathrm{C}$ & -2.981867 & 0.579734 & 4.382418 \\
$\mathrm{H}$ & -0.954063 & 0.601675 & 5.102587 \\
$\mathrm{H}$ & -4.873478 & 0.657267 & 3.360753 \\
$\mathrm{H}$ & -5.098272 & 1.818366 & -3.746401 \\
$\mathrm{C}$ & -3.076609 & -3.475691 & -0.491998 \\
$\mathrm{~N}$ & -4.607740 & -1.676279 & 0.172513 \\
$\mathrm{C}$ & -1.220279 & -3.071686 & -1.907231 \\
$\mathrm{H}$ & -0.979680 & -1.075597 & -2.619728 \\
$\mathrm{H}$ & 1.089481 & 6.315802 & 1.280639 \\
$\mathrm{H}$ & -3.420525 & 0.473912 & 5.366065 \\
$\mathrm{C}$ & -1.953239 & -3.928480 & -1.184958 \\
$\mathrm{H}$ & -3.667157 & -4.183255 & 0.075011 \\
$\mathrm{C}$ & -4.756295 & -2.066190 & 1.576065 \\
$\mathrm{C}$ & -5.824548 & -1.321921 & -0.559660 \\
$\mathrm{H}$ & -0.368430 & -3.436057 & -2.462656 \\
$\mathrm{H}$ & -1.680214 & -4.975295 & -1.145043 \\
$\mathrm{H}$ & -5.442458 & -1.366005 & 2.095736 \\
$\mathrm{H}$ & -5.183777 & -3.088767 & 1.641090 \\
$\mathrm{H}$ & -3.770801 & -2.051886 & 2.089402 \\
$\mathrm{H}$ & -6.699518 & -1.869585 & -0.148054 \\
$\mathrm{H}$ & -6.007687 & -0.231095 & -0.468258 \\
$\mathrm{H}$ & -5.743771 & -1.602767 & -1.629054 \\
$\mathrm{H}$ & 2.002563 & -1.051962 & -3.060312 \\
$\mathrm{~F}$ & 3.015969 & -5.018043 & -1.139094 \\
$\mathrm{H}$ & 2.003313 & -3.576882 & -3.057767 \\
$\mathrm{~F}$ & 4.082057 & -3.703882 & 0.915993 \\
\hline \hline & & & \\
\hline
\end{tabular}

Table S37: Cartesian coordinates $(\AA)$ of t2lTS $(\mathrm{E}=$ 1487.184419385190 a.u.).

\begin{tabular}{lrrr}
\hline \hline $\mathrm{C}$ & 0.116308 & -2.882168 & 2.731334 \\
$\mathrm{C}$ & 0.107477 & -2.097151 & 1.573586 \\
$\mathrm{C}$ & 0.959745 & -0.995862 & 1.418956 \\
$\mathrm{C}$ & 1.854832 & -0.690519 & 2.463052 \\
$\mathrm{C}$ & 1.850189 & -1.477343 & 3.625134 \\
$\mathrm{C}$ & 0.989125 & -2.565923 & 3.770570 \\
$\mathrm{Pd}$ & 0.820864 & 0.174644 & -0.253457 \\
$\mathrm{C}$ & 2.865590 & 0.417479 & 2.307167 \\
$\mathrm{C}$ & 4.124762 & -0.062390 & 1.546019 \\
$\mathrm{C}$ & 3.838778 & -0.839747 & 0.280502 \\
$\mathrm{C}$ & 2.949401 & -0.341749 & -0.717958 \\
$\mathrm{C}$ & 2.834655 & -1.058712 & -1.931493 \\
$\mathrm{C}$ & 3.519997 & -2.254257 & -2.160898 \\
$\mathrm{C}$ & 4.353760 & -2.728353 & -1.161393
\end{tabular}




\begin{tabular}{|c|c|c|c|}
\hline C & 4.521208 & -2.024065 & 0.032573 \\
\hline $\mathrm{H}$ & -0.605193 & -2.335212 & 0.796153 \\
\hline $\mathrm{H}$ & 2.541264 & -1.225821 & 4.429026 \\
\hline $\mathrm{H}$ & 1.001140 & -3.156389 & 4.684396 \\
\hline $\mathrm{H}$ & -0.567539 & -3.724964 & 2.817093 \\
\hline $\mathrm{H}$ & 3.114270 & 0.972992 & -0.853403 \\
\hline $\mathrm{H}$ & 3.185979 & 0.792676 & 3.287372 \\
\hline $\mathrm{H}$ & 2.439821 & 1.268494 & 1.771562 \\
\hline $\mathrm{H}$ & 4.747342 & -0.671904 & 2.208136 \\
\hline $\mathrm{H}$ & 4.686737 & 0.841714 & 1.275419 \\
\hline $\mathrm{Br}$ & 0.399742 & 1.684623 & -2.470099 \\
\hline $\mathrm{O}$ & 3.597438 & 2.121893 & -1.211207 \\
\hline $\mathrm{C}$ & 3.595944 & 3.098302 & -0.358021 \\
\hline $\mathrm{O}$ & 3.893469 & 3.093834 & 0.832917 \\
\hline $\mathrm{O}$ & 3.206703 & 4.293265 & -0.932737 \\
\hline $\mathrm{H}$ & 2.873055 & 4.032821 & -1.809136 \\
\hline $\mathrm{P}$ & -1.358262 & 0.924417 & 0.261424 \\
\hline $\mathrm{C}$ & -2.566106 & 0.821750 & -1.132180 \\
\hline C & -1.202318 & 2.682992 & 0.730979 \\
\hline $\mathrm{C}$ & -2.209690 & 0.304132 & 1.761923 \\
\hline $\mathrm{C}$ & -2.832590 & -0.403903 & -1.814148 \\
\hline $\mathrm{C}$ & -3.213877 & 1.994629 & -1.582729 \\
\hline $\mathrm{C}$ & -2.290752 & 3.390419 & 1.280554 \\
\hline $\mathrm{C}$ & 0.002398 & 3.284923 & 0.646678 \\
\hline $\mathrm{C}$ & -1.561990 & 0.347671 & 2.945946 \\
\hline $\mathrm{C}$ & -3.565473 & -0.071732 & 1.724452 \\
\hline $\mathrm{C}$ & -3.701710 & -0.385967 & -2.917930 \\
\hline $\mathrm{C}$ & -2.100674 & -1.671990 & -1.560350 \\
\hline $\mathrm{C}$ & -4.076047 & 1.973855 & -2.609573 \\
\hline $\mathrm{H}$ & -3.018064 & 2.951011 & -1.131033 \\
\hline $\mathrm{H}$ & -3.260049 & 2.921166 & 1.389645 \\
\hline C & -2.116099 & 4.719748 & 1.682813 \\
\hline $\mathrm{C}$ & 0.168697 & 4.563772 & 1.038297 \\
\hline $\mathrm{H}$ & 0.855081 & 2.736892 & 0.271215 \\
\hline $\mathrm{H}$ & -0.542485 & 0.704788 & 2.996939 \\
\hline $\mathrm{C}$ & -2.195851 & -0.036613 & 4.133701 \\
\hline $\mathrm{C}$ & -4.229088 & -0.429391 & 2.903236 \\
\hline $\mathrm{H}$ & -4.122247 & -0.064553 & 0.798848 \\
\hline $\mathrm{C}$ & -4.340050 & 0.791450 & -3.295357 \\
\hline $\mathrm{H}$ & -3.858502 & -1.276792 & -3.512234 \\
\hline $\mathrm{C}$ & -2.709081 & -2.793450 & -0.926836 \\
\hline $\mathrm{C}$ & -0.871587 & -1.815972 & -2.104976 \\
\hline $\mathrm{H}$ & -4.555098 & 2.891321 & -2.928017 \\
\hline $\mathrm{C}$ & -0.909403 & 5.296246 & 1.560324 \\
\hline $\mathrm{H}$ & -2.948007 & 5.276635 & 2.094579 \\
\hline $\mathrm{H}$ & 1.142997 & 5.028841 & 0.959668 \\
\hline $\mathrm{C}$ & -3.535249 & -0.428669 & 4.113275 \\
\hline $\mathrm{H}$ & -1.653942 & -0.008076 & 5.070126 \\
\hline $\mathrm{H}$ & -5.275312 & -0.704564 & 2.877876 \\
\hline $\mathrm{H}$ & -5.014396 & 0.795054 & -4.141998 \\
\hline C & -1.997897 & -4.006964 & -0.871298 \\
\hline
\end{tabular}




\begin{tabular}{lrrr}
$\mathrm{N}$ & -4.017488 & -2.721641 & -0.327815 \\
$\mathrm{C}$ & -0.178825 & -3.032700 & -2.036080 \\
$\mathrm{H}$ & -0.416550 & -1.001446 & -2.647407 \\
$\mathrm{H}$ & -0.764319 & 6.322544 & 1.872629 \\
$\mathrm{H}$ & -4.035622 & -0.711976 & 5.030078 \\
$\mathrm{C}$ & -0.725075 & -4.098409 & -1.436570 \\
$\mathrm{H}$ & -2.430767 & -4.880340 & -0.401434 \\
$\mathrm{C}$ & -4.194510 & -3.217161 & 1.038566 \\
$\mathrm{C}$ & -5.204932 & -2.658463 & -1.180945 \\
$\mathrm{H}$ & 0.797405 & -3.119468 & -2.492317 \\
$\mathrm{H}$ & -0.182930 & -5.034552 & -1.396433 \\
$\mathrm{H}$ & -5.093861 & -2.757106 & 1.497679 \\
$\mathrm{H}$ & -4.331422 & -4.318835 & 1.026317 \\
$\mathrm{H}$ & -3.306922 & -2.969010 & 1.659485 \\
$\mathrm{H}$ & -5.932189 & -3.447928 & -0.893321 \\
$\mathrm{H}$ & -5.688618 & -1.665736 & -1.069843 \\
$\mathrm{H}$ & -4.943353 & -2.833318 & -2.244006 \\
$\mathrm{H}$ & 2.208395 & -0.645639 & -2.716831 \\
$\mathrm{~F}$ & 5.045373 & -3.876067 & -1.334412 \\
$\mathrm{H}$ & 3.429581 & -2.812164 & -3.088478 \\
$\mathrm{~F}$ & 5.392973 & -2.541683 & 0.936666 \\
\hline \hline
\end{tabular}

Table S38: Cartesian coordinates $(\AA)$ of $\mathbf{t 2 m R}(\mathrm{E}=$ 1748.317450827058 a.u.).

\begin{tabular}{lrrr}
\hline \hline $\mathrm{C}$ & -1.011491 & -1.961363 & 3.539603 \\
$\mathrm{C}$ & -0.770983 & -1.599424 & 2.209676 \\
$\mathrm{C}$ & 0.403163 & -0.936612 & 1.836949 \\
$\mathrm{C}$ & 1.356306 & -0.608495 & 2.822210 \\
$\mathrm{C}$ & 1.095197 & -0.968493 & 4.154050 \\
$\mathrm{C}$ & -0.074492 & -1.636922 & 4.519415 \\
$\mathrm{Pd}$ & 0.751903 & -0.378874 & -0.095866 \\
$\mathrm{C}$ & 2.666778 & 0.035763 & 2.449542 \\
$\mathrm{C}$ & 3.717758 & -1.015519 & 1.993304 \\
$\mathrm{C}$ & 3.174663 & -2.044901 & 1.034775 \\
$\mathrm{C}$ & 3.074583 & -1.757403 & -0.350552 \\
$\mathrm{C}$ & 2.700533 & -2.799307 & -1.224641 \\
$\mathrm{C}$ & 2.343997 & -4.062562 & -0.757074 \\
$\mathrm{C}$ & 2.383937 & -4.308059 & 0.616567 \\
$\mathrm{C}$ & 2.804939 & -3.317779 & 1.497870 \\
$\mathrm{H}$ & -1.526939 & -1.820386 & 1.468172 \\
$\mathrm{H}$ & 2.864834 & -3.527970 & 2.562262 \\
$\mathrm{H}$ & 1.830071 & -0.709349 & 4.915041 \\
$\mathrm{H}$ & -0.254324 & -1.893047 & 5.561464 \\
$\mathrm{H}$ & -1.933320 & -2.477611 & 3.800949 \\
$\mathrm{H}$ & 3.505783 & -0.822321 & -0.717690 \\
$\mathrm{H}$ & 3.088981 & 0.595179 & 3.290693 \\
$\mathrm{H}$ & 2.559999 & 0.766427 & 1.643467 \\
$\mathrm{H}$ & 4.100525 & -1.540558 & 2.878918 \\
$\mathrm{H}$ & 4.525285 & -0.458229 & 1.500867 \\
$\mathrm{Br}$ & 1.067802 & 0.528302 & -2.574142
\end{tabular}




\begin{tabular}{|c|c|c|c|}
\hline $\mathrm{O}$ & 5.052360 & 0.633917 & -0.266149 \\
\hline $\mathrm{C}$ & 4.928313 & 1.813305 & 0.178918 \\
\hline $\mathrm{O}$ & 4.464788 & 2.230469 & 1.252633 \\
\hline $\mathrm{O}$ & 5.411715 & 2.812192 & -0.700937 \\
\hline $\mathrm{H}$ & 5.685809 & 2.289825 & -1.472392 \\
\hline $\mathrm{P}$ & -0.875097 & 1.241497 & -0.049657 \\
\hline $\mathrm{C}$ & -2.010712 & 1.181531 & -1.503723 \\
\hline $\mathrm{C}$ & -0.063949 & 2.879512 & -0.033757 \\
\hline $\mathrm{C}$ & -1.926708 & 1.413742 & 1.442691 \\
\hline $\mathrm{C}$ & -2.700922 & -0.010504 & -1.882238 \\
\hline $\mathrm{C}$ & -2.167298 & 2.331940 & -2.310186 \\
\hline $\mathrm{C}$ & -0.815050 & 4.051361 & 0.192010 \\
\hline $\mathrm{C}$ & 1.278548 & 2.968416 & -0.147768 \\
\hline $\mathrm{C}$ & -1.334765 & 1.608981 & 2.640948 \\
\hline $\mathrm{C}$ & -3.327856 & 1.497923 & 1.339264 \\
\hline $\mathrm{C}$ & -3.473798 & 0.003079 & -3.055606 \\
\hline $\mathrm{C}$ & -2.507663 & -1.330261 & -1.225952 \\
\hline $\mathrm{C}$ & -2.951482 & 2.329708 & -3.397842 \\
\hline $\mathrm{H}$ & -1.641876 & 3.244656 & -2.090947 \\
\hline $\mathrm{H}$ & -1.889458 & 4.006838 & 0.316201 \\
\hline $\mathrm{C}$ & -0.164704 & 5.289291 & 0.245917 \\
\hline $\mathrm{C}$ & 1.901718 & 4.162866 & -0.095122 \\
\hline $\mathrm{H}$ & 1.873946 & 2.075622 & -0.278054 \\
\hline $\mathrm{H}$ & -0.256534 & 1.624635 & 2.720145 \\
\hline $\mathrm{C}$ & -2.092197 & 1.826813 & 3.797997 \\
\hline $\mathrm{C}$ & -4.104101 & 1.746096 & 2.476792 \\
\hline $\mathrm{H}$ & -3.825038 & 1.402617 & 0.385373 \\
\hline $\mathrm{C}$ & -3.618900 & 1.173912 & -3.793228 \\
\hline $\mathrm{H}$ & -3.935873 & -0.903701 & -3.423331 \\
\hline $\mathrm{C}$ & -3.519638 & -1.946386 & -0.434358 \\
\hline $\mathrm{C}$ & -1.403744 & -2.043117 & -1.543914 \\
\hline $\mathrm{H}$ & -3.048306 & 3.229325 & -3.992847 \\
\hline $\mathrm{C}$ & 1.168642 & 5.343718 & 0.100871 \\
\hline $\mathrm{H}$ & -0.734086 & 6.195829 & 0.406354 \\
\hline $\mathrm{H}$ & 2.977458 & 4.215231 & -0.198068 \\
\hline $\mathrm{C}$ & -3.483734 & 1.893767 & 3.717108 \\
\hline $\mathrm{H}$ & -1.597550 & 1.966437 & 4.750454 \\
\hline $\mathrm{H}$ & -5.180057 & 1.826881 & 2.395989 \\
\hline $\mathrm{H}$ & -4.222912 & 1.177310 & -4.691426 \\
\hline $\mathrm{C}$ & -3.321331 & -3.267085 & 0.009998 \\
\hline $\mathrm{N}$ & -4.730554 & -1.259857 & -0.063355 \\
\hline $\mathrm{C}$ & -1.224790 & -3.356408 & -1.089415 \\
\hline $\mathrm{H}$ & -0.655474 & -1.628356 & -2.201014 \\
\hline $\mathrm{H}$ & 1.680428 & 6.296745 & 0.142675 \\
\hline $\mathrm{H}$ & -4.074462 & 2.078104 & 4.604918 \\
\hline $\mathrm{C}$ & -2.155035 & -3.952426 & -0.332790 \\
\hline $\mathrm{H}$ & -4.070858 & -3.766589 & 0.609822 \\
\hline $\mathrm{C}$ & -5.129553 & -1.250166 & 1.345310 \\
\hline $\mathrm{C}$ & -5.780487 & -1.050305 & -1.061230 \\
\hline $\mathrm{H}$ & -0.339110 & -3.904128 & -1.376283 \\
\hline $\mathrm{H}$ & -2.008071 & -4.970383 & 0.004983 \\
\hline
\end{tabular}




\begin{tabular}{lrrr}
$\mathrm{H}$ & -5.801212 & -0.389774 & 1.544790 \\
$\mathrm{H}$ & -5.674453 & -2.186057 & 1.590076 \\
$\mathrm{H}$ & -4.236655 & -1.169876 & 2.001849 \\
$\mathrm{H}$ & -6.756211 & -1.424636 & -0.683376 \\
$\mathrm{H}$ & -5.871905 & 0.033737 & -1.280822 \\
$\mathrm{H}$ & -5.561881 & -1.607021 & -1.994830 \\
$\mathrm{H}$ & 2.068543 & -4.842372 & -1.459369 \\
$\mathrm{H}$ & 2.105542 & -5.290283 & 0.992095 \\
$\mathrm{Cl}$ & 2.817411 & -2.584737 & -2.961924 \\
\hline \hline
\end{tabular}

Table S39: Cartesian coordinates $(\AA)$ of t2mTS $(\mathrm{E}=$ 1748.302964936718 a.u.).

\begin{tabular}{lrrr}
\hline $\mathrm{C}$ & -0.622782 & -2.635373 & 3.074651 \\
$\mathrm{C}$ & -0.374425 & -2.030834 & 1.837105 \\
$\mathrm{C}$ & 0.682186 & -1.129499 & 1.657837 \\
$\mathrm{C}$ & 1.509851 & -0.835658 & 2.759846 \\
$\mathrm{C}$ & 1.246882 & -1.438566 & 3.998657 \\
$\mathrm{C}$ & 0.188267 & -2.332805 & 4.166600 \\
$\mathrm{Pd}$ & 0.955689 & -0.149087 & -0.116088 \\
$\mathrm{C}$ & 2.720444 & 0.045595 & 2.583865 \\
$\mathrm{C}$ & 3.914485 & -0.758882 & 2.012330 \\
$\mathrm{C}$ & 3.560135 & -1.745265 & 0.918723 \\
$\mathrm{C}$ & 2.976718 & -1.298310 & -0.308225 \\
$\mathrm{C}$ & 2.885524 & -2.274912 & -1.323449 \\
$\mathrm{C}$ & 3.199636 & -3.623336 & -1.138367 \\
$\mathrm{C}$ & 3.684378 & -4.030201 & 0.100847 \\
$\mathrm{C}$ & 3.890175 & -3.089898 & 1.110621 \\
$\mathrm{H}$ & -1.045025 & -2.249687 & 1.017265 \\
$\mathrm{H}$ & 4.317457 & -3.404717 & 2.060250 \\
$\mathrm{H}$ & 1.889508 & -1.197126 & 4.844820 \\
$\mathrm{H}$ & -0.001076 & -2.781985 & 5.139371 \\
$\mathrm{H}$ & -1.457918 & -3.325997 & 3.178374 \\
$\mathrm{H}$ & 3.446868 & -0.103129 & -0.666832 \\
$\mathrm{H}$ & 3.025050 & 0.491386 & 3.539175 \\
$\mathrm{H}$ & 2.505670 & 0.874781 & 1.905934 \\
$\mathrm{H}$ & 4.393463 & -1.312037 & 2.830831 \\
$\mathrm{H}$ & 4.632632 & -0.025731 & 1.621664 \\
$\mathrm{Br}$ & 0.967501 & 1.403261 & -2.378888 \\
$\mathrm{O}$ & 4.156062 & 0.859855 & -1.125229 \\
$\mathrm{C}$ & 4.279676 & 1.940552 & -0.413569 \\
$\mathrm{O}$ & 4.689689 & 2.060471 & 0.739240 \\
$\mathrm{O}$ & 3.899986 & 3.073473 & -1.095945 \\
$\mathrm{H}$ & 3.392352 & 2.735734 & -1.859054 \\
$\mathrm{P}$ & -1.004712 & 1.134218 & 0.210265 \\
$\mathrm{C}$ & -2.134958 & 1.197600 & -1.249317 \\
$\mathrm{C}$ & -0.486511 & 2.845074 & 0.591633 \\
$\mathrm{C}$ & -2.054714 & 0.829471 & 1.685235 \\
$\mathrm{C}$ & -2.649710 & 0.014982 & -1.862702 \\
$\mathrm{C}$ & -2.461188 & 2.447692 & -1.823456 \\
$\mathrm{C}$ & -1.423901 & 3.812661 & 1.007193
\end{tabular}




\begin{tabular}{|c|c|c|c|}
\hline C & 0.825287 & 3.159974 & 0.579670 \\
\hline $\mathrm{C}$ & -1.489672 & 0.881472 & 2.910879 \\
\hline $\mathrm{C}$ & -3.451147 & 0.696823 & 1.570861 \\
\hline $\mathrm{C}$ & -3.425820 & 0.144285 & -3.026783 \\
\hline $\mathrm{C}$ & -2.261301 & -1.364362 & -1.466365 \\
\hline $\mathrm{C}$ & -3.247168 & 2.543438 & -2.905452 \\
\hline $\mathrm{H}$ & -2.065754 & 3.366094 & -1.426752 \\
\hline $\mathrm{H}$ & -2.479060 & 3.576569 & 1.059695 \\
\hline $\mathrm{C}$ & -0.983918 & 5.096573 & 1.349965 \\
\hline $\mathrm{C}$ & 1.247020 & 4.395645 & 0.913374 \\
\hline $\mathrm{H}$ & 1.556792 & 2.412968 & 0.309181 \\
\hline $\mathrm{H}$ & -0.429351 & 1.064351 & 3.014363 \\
\hline $\mathrm{C}$ & -2.258155 & 0.737041 & 4.072022 \\
\hline $\mathrm{C}$ & -4.243833 & 0.582648 & 2.718617 \\
\hline $\mathrm{H}$ & -3.937076 & 0.711169 & 0.606703 \\
\hline $\mathrm{C}$ & -3.745243 & 1.402437 & -3.527838 \\
\hline $\mathrm{H}$ & -3.751381 & -0.730064 & -3.574054 \\
\hline $\mathrm{C}$ & -3.181198 & -2.277406 & -0.874586 \\
\hline $\mathrm{C}$ & -1.043478 & -1.815602 & -1.841177 \\
\hline $\mathrm{H}$ & -3.476107 & 3.517597 & -3.319272 \\
\hline $\mathrm{C}$ & 0.327085 & 5.383208 & 1.300059 \\
\hline $\mathrm{H}$ & -1.696428 & 5.850910 & 1.658251 \\
\hline $\mathrm{H}$ & 2.304333 & 4.627445 & 0.892732 \\
\hline $\mathrm{C}$ & -3.641811 & 0.584386 & 3.976579 \\
\hline $\mathrm{H}$ & -1.782093 & 0.767577 & 5.043519 \\
\hline $\mathrm{H}$ & -5.319057 & 0.498320 & 2.633295 \\
\hline $\mathrm{H}$ & -4.350437 & 1.492689 & -4.420682 \\
\hline $\mathrm{C}$ & -2.783674 & -3.613268 & -0.684979 \\
\hline $\mathrm{N}$ & -4.499025 & -1.877670 & -0.452982 \\
\hline $\mathrm{C}$ & -0.667635 & -3.153351 & -1.645570 \\
\hline $\mathrm{H}$ & -0.350282 & -1.155548 & -2.340953 \\
\hline $\mathrm{H}$ & 0.676737 & 6.372286 & 1.567138 \\
\hline $\mathrm{H}$ & -4.243983 & 0.488988 & 4.870706 \\
\hline $\mathrm{C}$ & -1.512953 & -4.027756 & -1.084879 \\
\hline $\mathrm{H}$ & -3.460215 & -4.332133 & -0.241188 \\
\hline $\mathrm{C}$ & -4.918587 & -2.181008 & 0.916207 \\
\hline $\mathrm{C}$ & -5.552896 & -1.689602 & -1.451077 \\
\hline $\mathrm{H}$ & 0.307085 & -3.491265 & -1.966507 \\
\hline $\mathrm{H}$ & -1.212438 & -5.058211 & -0.944246 \\
\hline $\mathrm{H}$ & -5.702148 & -1.465106 & 1.239710 \\
\hline $\mathrm{H}$ & -5.336328 & -3.208727 & 0.963872 \\
\hline $\mathrm{H}$ & -4.055794 & -2.112670 & 1.613085 \\
\hline $\mathrm{H}$ & -6.451714 & -2.286670 & -1.185370 \\
\hline $\mathrm{H}$ & -5.834847 & -0.617095 & -1.494681 \\
\hline $\mathrm{H}$ & -5.222205 & -2.034144 & -2.451923 \\
\hline $\mathrm{H}$ & 3.085126 & -4.327264 & -1.956541 \\
\hline $\mathrm{H}$ & 3.932150 & -5.076661 & 0.267417 \\
\hline $\mathrm{Cl}$ & 2.461343 & -1.823707 & -2.980549 \\
\hline
\end{tabular}


Table S40: Cartesian coordinates $(\AA)$ of t2nR (E= 1748.329475295138 a.u.).

\begin{tabular}{|c|c|c|c|}
\hline $\mathrm{C}$ & 0.725094 & 1.483196 & 3.755747 \\
\hline $\mathrm{C}$ & 0.577515 & 1.239691 & 2.386593 \\
\hline $\mathrm{C}$ & -0.594942 & 0.679568 & 1.862758 \\
\hline $\mathrm{C}$ & -1.634620 & 0.316338 & 2.742815 \\
\hline $\mathrm{C}$ & -1.465788 & 0.559550 & 4.116604 \\
\hline $\mathrm{C}$ & -0.305360 & 1.139240 & 4.628403 \\
\hline $\mathrm{Pd}$ & -0.773727 & 0.344303 & -0.150724 \\
\hline $\mathrm{C}$ & -2.933522 & -0.269761 & 2.249059 \\
\hline $\mathrm{C}$ & -3.956010 & 0.829031 & 1.848657 \\
\hline $\mathrm{C}$ & -3.369493 & 1.873350 & 0.941224 \\
\hline $\mathrm{C}$ & -2.961086 & 1.516343 & -0.368536 \\
\hline $\mathrm{C}$ & -2.450619 & 2.495463 & -1.246599 \\
\hline $\mathrm{C}$ & -2.349718 & 3.815445 & -0.812826 \\
\hline $\mathrm{C}$ & -2.722393 & 4.178496 & 0.478687 \\
\hline $\mathrm{C}$ & -3.219843 & 3.203535 & 1.346249 \\
\hline $\mathrm{H}$ & 1.414664 & 1.459037 & 1.737458 \\
\hline $\mathrm{H}$ & -3.514389 & 3.491291 & 2.352615 \\
\hline $\mathrm{H}$ & -2.269479 & 0.273599 & 4.793628 \\
\hline $\mathrm{H}$ & -0.202895 & 1.305307 & 5.698735 \\
\hline $\mathrm{H}$ & 1.648709 & 1.921419 & 4.128943 \\
\hline $\mathrm{H}$ & -3.276883 & 0.536700 & -0.747949 \\
\hline $\mathrm{H}$ & -3.395859 & -0.897776 & 3.017408 \\
\hline $\mathrm{H}$ & -2.797199 & -0.929821 & 1.389255 \\
\hline $\mathrm{H}$ & -4.351762 & 1.312835 & 2.751517 \\
\hline $\mathrm{H}$ & -4.759545 & 0.311099 & 1.308135 \\
\hline $\mathrm{Br}$ & -0.872423 & -0.213171 & -2.785486 \\
\hline $\mathrm{O}$ & -4.950391 & -0.805756 & -0.596169 \\
\hline $\mathrm{C}$ & -4.995750 & -1.998541 & -0.170045 \\
\hline $\mathrm{O}$ & -4.567077 & -2.503755 & 0.878746 \\
\hline $\mathrm{O}$ & -5.657667 & -2.892779 & -1.047549 \\
\hline $\mathrm{H}$ & -5.882418 & -2.315634 & -1.795596 \\
\hline $\mathrm{P}$ & 0.839768 & -1.297976 & -0.152327 \\
\hline $\mathrm{C}$ & 2.083479 & -1.094400 & -1.501660 \\
\hline $\mathrm{C}$ & -0.000114 & -2.899813 & -0.404982 \\
\hline $\mathrm{C}$ & 1.753253 & -1.670557 & 1.390898 \\
\hline $\mathrm{C}$ & 2.817926 & 0.117527 & -1.683947 \\
\hline $\mathrm{C}$ & 2.266629 & -2.136932 & -2.438888 \\
\hline $\mathrm{C}$ & 0.704241 & -4.112584 & -0.261702 \\
\hline $\mathrm{C}$ & -1.327918 & -2.931117 & -0.647593 \\
\hline $\mathrm{C}$ & 1.055247 & -1.982260 & 2.504481 \\
\hline $\mathrm{C}$ & 3.156151 & -1.773356 & 1.398329 \\
\hline $\mathrm{C}$ & 3.647654 & 0.237765 & -2.811244 \\
\hline $\mathrm{C}$ & 2.642185 & 1.333664 & -0.846245 \\
\hline $\mathrm{C}$ & 3.107737 & -2.013849 & -3.475766 \\
\hline $\mathrm{H}$ & 1.715793 & -3.058122 & -2.366346 \\
\hline $\mathrm{H}$ & 1.763775 & -4.116279 & -0.039869 \\
\hline $\mathrm{C}$ & 0.026837 & -5.326862 & -0.419800 \\
\hline $\mathrm{C}$ & -1.976626 & -4.103027 & -0.800993 \\
\hline
\end{tabular}




\begin{tabular}{lrrr}
$\mathrm{H}$ & -1.890506 & -2.009749 & -0.716962 \\
$\mathrm{H}$ & -0.026332 & -1.974537 & 2.490264 \\
$\mathrm{C}$ & 1.705281 & -2.346348 & 3.689677 \\
$\mathrm{C}$ & 3.826459 & -2.163524 & 2.563389 \\
$\mathrm{H}$ & 3.734072 & -1.582109 & 0.506043 \\
$\mathrm{C}$ & 3.810663 & -0.831223 & -3.686860 \\
$\mathrm{H}$ & 4.142879 & 1.172625 & -3.035817 \\
$\mathrm{C}$ & 3.665855 & 1.812628 & 0.020960 \\
$\mathrm{C}$ & 1.538963 & 2.092766 & -1.034006 \\
$\mathrm{H}$ & 3.222094 & -2.832352 & -4.175507 \\
$\mathrm{C}$ & -1.288400 & -5.321377 & -0.688760 \\
$\mathrm{H}$ & 0.560706 & -6.263601 & -0.323596 \\
$\mathrm{H}$ & -3.038645 & -4.108900 & -1.006928 \\
$\mathrm{C}$ & 3.097616 & -2.435823 & 3.721038 \\
$\mathrm{H}$ & 1.127333 & -2.578489 & 4.574801 \\
$\mathrm{H}$ & 4.904112 & -2.258698 & 2.567653 \\
$\mathrm{H}$ & 4.459712 & -0.732865 & -4.547468 \\
$\mathrm{C}$ & 3.481101 & 3.043172 & 0.675491 \\
$\mathrm{~N}$ & 4.883037 & 1.079410 & 0.253237 \\
$\mathrm{C}$ & 1.373693 & 3.317789 & -0.371596 \\
$\mathrm{H}$ & 0.774786 & 1.784595 & -1.729885 \\
$\mathrm{H}$ & -1.820856 & -6.255831 & -0.811916 \\
$\mathrm{H}$ & 3.605741 & -2.731422 & 4.629671 \\
$\mathrm{C}$ & 2.316908 & 3.781294 & 0.458985 \\
$\mathrm{H}$ & 4.241626 & 3.432120 & 1.340454 \\
$\mathrm{C}$ & 5.263408 & 0.765299 & 1.630802 \\
$\mathrm{C}$ & 5.951337 & 1.112583 & -0.746625 \\
$\mathrm{H}$ & 0.481196 & 3.901062 & -0.539816 \\
$\mathrm{H}$ & 2.178175 & 4.730111 & 0.961326 \\
$\mathrm{H}$ & 5.832088 & -0.187122 & 1.655753 \\
$\mathrm{H}$ & 5.904091 & 1.575326 & 2.039014 \\
$\mathrm{H}$ & 4.363108 & 0.667970 & 2.274608 \\
$\mathrm{H}$ & 6.907584 & 1.437590 & -0.283230 \\
$\mathrm{H}$ & 6.087972 & 0.098807 & -1.177050 \\
$\mathrm{H}$ & 5.721324 & 1.837156 & -1.554341 \\
$\mathrm{H}$ & -2.629193 & 5.211110 & 0.801079 \\
$\mathrm{Cl}$ & -1.780176 & 5.065736 & -1.929601 \\
$\mathrm{H}$ & -2.181222 & 2.216889 & -2.258517 \\
\hline \hline & & & \\
\hline
\end{tabular}

Table S41: Cartesian coordinates $(\AA)$ of t2nTS $(\mathrm{E}=$ 1748.316303457819 a.u.).

\begin{tabular}{lrrr}
\hline \hline $\mathrm{C}$ & -0.486937 & -1.898276 & 3.577983 \\
$\mathrm{C}$ & -0.273045 & -1.497118 & 2.255074 \\
$\mathrm{C}$ & 0.710860 & -0.559183 & 1.914187 \\
$\mathrm{C}$ & 1.504586 & -0.019508 & 2.945711 \\
$\mathrm{C}$ & 1.275744 & -0.419098 & 4.271504 \\
$\mathrm{C}$ & 0.288614 & -1.350006 & 4.597586 \\
$\mathrm{Pd}$ & 0.903372 & 0.067895 & -0.025891 \\
$\mathrm{C}$ & 2.641706 & 0.917617 & 2.628518 \\
$\mathrm{C}$ & 3.919672 & 0.146208 & 2.223053
\end{tabular}




\begin{tabular}{|c|c|c|c|}
\hline C & 3.722776 & -0.947896 & 1.196572 \\
\hline $\mathrm{C}$ & 3.022555 & -0.692933 & -0.016357 \\
\hline $\mathrm{C}$ & 3.009783 & -1.703266 & -1.007649 \\
\hline $\mathrm{C}$ & 3.628204 & -2.928558 & -0.776912 \\
\hline $\mathrm{C}$ & 4.277198 & -3.192954 & 0.425418 \\
\hline $\mathrm{C}$ & 4.326264 & -2.192430 & 1.399095 \\
\hline $\mathrm{H}$ & -0.918722 & -1.900988 & 1.487535 \\
\hline $\mathrm{H}$ & 4.857595 & -2.388888 & 2.328773 \\
\hline $\mathrm{H}$ & 1.889609 & 0.014298 & 5.060643 \\
\hline $\mathrm{H}$ & 0.126516 & -1.637712 & 5.634405 \\
\hline $\mathrm{H}$ & -1.267434 & -2.623467 & 3.801979 \\
\hline $\mathrm{H}$ & 3.301198 & 0.522244 & -0.467752 \\
\hline $\mathrm{H}$ & 2.883813 & 1.544484 & 3.496186 \\
\hline $\mathrm{H}$ & 2.379087 & 1.600198 & 1.817706 \\
\hline $\mathrm{H}$ & 4.384078 & -0.288533 & 3.118511 \\
\hline $\mathrm{H}$ & 4.609544 & 0.894451 & 1.809106 \\
\hline $\mathrm{Br}$ & 0.921969 & 0.879589 & -2.611105 \\
\hline $\mathrm{O}$ & 3.946517 & 1.500150 & -1.049208 \\
\hline $\mathrm{C}$ & 3.965049 & 2.676441 & -0.506235 \\
\hline $\mathrm{O}$ & 4.099989 & 2.992137 & 0.672532 \\
\hline $\mathrm{O}$ & 3.821296 & 3.684354 & -1.441787 \\
\hline $\mathrm{H}$ & 3.587250 & 3.211850 & -2.259644 \\
\hline $\mathrm{P}$ & -1.227854 & 1.070906 & -0.077509 \\
\hline $\mathrm{C}$ & -2.251299 & 0.630728 & -1.550375 \\
\hline $\mathrm{C}$ & -0.951543 & 2.876089 & -0.112875 \\
\hline $\mathrm{C}$ & -2.319533 & 0.978109 & 1.392106 \\
\hline $\mathrm{C}$ & -2.553217 & -0.724169 & -1.884423 \\
\hline C & -2.703312 & 1.652120 & -2.416790 \\
\hline $\mathrm{C}$ & -2.022601 & 3.780345 & 0.035757 \\
\hline $\mathrm{C}$ & 0.309914 & 3.350539 & -0.179299 \\
\hline $\mathrm{C}$ & -1.825514 & 1.337040 & 2.596526 \\
\hline $\mathrm{C}$ & -3.689677 & 0.684808 & 1.261692 \\
\hline $\mathrm{C}$ & -3.253918 & -0.984732 & -3.073912 \\
\hline $\mathrm{C}$ & -2.012305 & -1.899853 & -1.153755 \\
\hline $\mathrm{C}$ & -3.413983 & 1.377358 & -3.520230 \\
\hline $\mathrm{H}$ & -2.469744 & 2.685990 & -2.233163 \\
\hline $\mathrm{H}$ & -3.041244 & 3.424964 & 0.123820 \\
\hline $\mathrm{C}$ & -1.764448 & 5.155899 & 0.061739 \\
\hline $\mathrm{C}$ & 0.555969 & 4.675328 & -0.150217 \\
\hline $\mathrm{H}$ & 1.142469 & 2.664347 & -0.245686 \\
\hline $\mathrm{H}$ & -0.789923 & 1.633224 & 2.694875 \\
\hline $\mathrm{C}$ & -2.639233 & 1.362421 & 3.735543 \\
\hline $\mathrm{C}$ & -4.528969 & 0.734218 & 2.380292 \\
\hline $\mathrm{H}$ & -4.120977 & 0.445964 & 0.300511 \\
\hline $\mathrm{C}$ & -3.704613 & 0.062158 & -3.872172 \\
\hline $\mathrm{H}$ & -3.420585 & -2.001053 & -3.405796 \\
\hline $\mathrm{C}$ & -2.832593 & -2.745504 & -0.353114 \\
\hline $\mathrm{C}$ & -0.732800 & -2.265819 & -1.392174 \\
\hline $\mathrm{H}$ & -3.744298 & 2.184696 & -4.161897 \\
\hline $\mathrm{C}$ & -0.498754 & 5.594693 & -0.033179 \\
\hline $\mathrm{H}$ & -2.580323 & 5.860077 & 0.162834 \\
\hline
\end{tabular}




\begin{tabular}{lrrr}
$\mathrm{H}$ & 1.575213 & 5.035276 & -0.207712 \\
$\mathrm{C}$ & -3.997230 & 1.058407 & 3.628409 \\
$\mathrm{H}$ & -2.219968 & 1.639051 & 4.694217 \\
$\mathrm{H}$ & -5.585596 & 0.524211 & 2.279074 \\
$\mathrm{H}$ & -4.251133 & -0.146976 & -4.782743 \\
$\mathrm{C}$ & -2.274837 & -3.922435 & 0.179798 \\
$\mathrm{~N}$ & -4.206486 & -2.428387 & -0.058096 \\
$\mathrm{C}$ & -0.195210 & -3.440958 & -0.848644 \\
$\mathrm{H}$ & -0.108760 & -1.675007 & -2.044768 \\
$\mathrm{H}$ & -0.289572 & 6.656566 & -0.009966 \\
$\mathrm{H}$ & -4.635044 & 1.091432 & 4.502062 \\
$\mathrm{C}$ & -0.943774 & -4.247664 & -0.085321 \\
$\mathrm{H}$ & -2.870884 & -4.588736 & 0.789705 \\
$\mathrm{C}$ & -4.651664 & -2.458260 & 1.336159 \\
$\mathrm{C}$ & -5.227700 & -2.590196 & -1.093841 \\
$\mathrm{H}$ & 0.828957 & -3.709638 & -1.062590 \\
$\mathrm{H}$ & -0.518412 & -5.155435 & 0.323217 \\
$\mathrm{H}$ & -5.539434 & -1.805263 & 1.465534 \\
$\mathrm{H}$ & -4.930934 & -3.494450 & 1.620996 \\
$\mathrm{H}$ & -3.842578 & -2.104847 & 2.010971 \\
$\mathrm{H}$ & -6.064851 & -3.217790 & -0.719412 \\
$\mathrm{H}$ & -5.625356 & -1.594584 & -1.380884 \\
$\mathrm{H}$ & -4.814752 & -3.102920 & -1.986014 \\
$\mathrm{H}$ & 4.750661 & -4.155882 & 0.592048 \\
$\mathrm{Cl}$ & 3.613758 & -4.184995 & -2.034005 \\
$\mathrm{H}$ & 2.536312 & -1.501292 & -1.963417 \\
\hline \hline & & &
\end{tabular}

Table S42: Cartesian coordinates $(\AA)$ of t2oR $(\mathrm{E}=$ 1748.331834118569 a.u.).

\begin{tabular}{lrrr}
\hline \hline $\mathrm{C}$ & 0.286929 & 2.483699 & 3.073746 \\
$\mathrm{C}$ & 0.243004 & 1.878558 & 1.813819 \\
$\mathrm{C}$ & -0.789168 & 1.003977 & 1.455212 \\
$\mathrm{C}$ & -1.800909 & 0.711290 & 2.392594 \\
$\mathrm{C}$ & -1.736263 & 1.320487 & 3.657285 \\
$\mathrm{C}$ & -0.708826 & 2.197353 & 4.005368 \\
$\mathrm{Pd}$ & -0.767789 & 0.133712 & -0.397733 \\
$\mathrm{C}$ & -2.972333 & -0.176911 & 2.054565 \\
$\mathrm{C}$ & -4.112892 & 0.609431 & 1.345928 \\
$\mathrm{C}$ & -3.597379 & 1.473406 & 0.230049 \\
$\mathrm{C}$ & -3.053519 & 0.862051 & -0.930655 \\
$\mathrm{C}$ & -2.562635 & 1.668627 & -1.977601 \\
$\mathrm{C}$ & -2.593778 & 3.064170 & -1.888280 \\
$\mathrm{C}$ & -3.121997 & 3.636238 & -0.736765 \\
$\mathrm{C}$ & -3.615888 & 2.867040 & 0.318057 \\
$\mathrm{H}$ & 1.045519 & 2.078738 & 1.118272 \\
$\mathrm{H}$ & -4.014922 & 3.354169 & 1.201872 \\
$\mathrm{H}$ & -2.515053 & 1.087971 & 4.382211 \\
$\mathrm{H}$ & -0.683968 & 2.644269 & 4.996932 \\
$\mathrm{H}$ & 1.103758 & 3.159297 & 3.319969 \\
$\mathrm{H}$ & -3.251127 & -0.206806 & -1.073146
\end{tabular}




\begin{tabular}{|c|c|c|c|}
\hline $\mathrm{H}$ & -3.388639 & -0.634705 & 2.957940 \\
\hline $\mathrm{H}$ & -2.695190 & -1.015600 & 1.411462 \\
\hline $\mathrm{H}$ & -4.643408 & 1.233086 & 2.077207 \\
\hline $\mathrm{H}$ & -4.787584 & -0.145382 & 0.920537 \\
\hline $\mathrm{Br}$ & -0.580693 & -1.087059 & -2.792536 \\
\hline $\mathrm{O}$ & -4.762557 & -1.683537 & -0.661585 \\
\hline $\mathrm{C}$ & -4.644235 & -2.724504 & 0.051202 \\
\hline $\mathrm{O}$ & -4.187529 & -2.879063 & 1.194520 \\
\hline $\mathrm{O}$ & -5.127072 & -3.901414 & -0.572715 \\
\hline $\mathrm{H}$ & -5.407018 & -3.571142 & -1.442034 \\
\hline $\mathrm{P}$ & 1.049033 & -1.203611 & 0.110142 \\
\hline $\mathrm{C}$ & 2.365201 & -1.187590 & -1.184880 \\
\hline $\mathrm{C}$ & 0.438278 & -2.918950 & 0.259353 \\
\hline $\mathrm{C}$ & 1.881781 & -1.034797 & 1.733560 \\
\hline $\mathrm{C}$ & 2.959543 & 0.024659 & -1.651631 \\
\hline $\mathrm{C}$ & 2.765741 & -2.404054 & -1.783725 \\
\hline $\mathrm{C}$ & 1.271920 & -3.940967 & 0.757922 \\
\hline $\mathrm{C}$ & -0.850299 & -3.199795 & -0.030093 \\
\hline $\mathrm{C}$ & 1.143789 & -1.131644 & 2.860348 \\
\hline $\mathrm{C}$ & 3.283934 & -0.958794 & 1.826218 \\
\hline $\mathrm{C}$ & 3.883615 & -0.041606 & -2.708071 \\
\hline $\mathrm{C}$ & 2.532279 & 1.383091 & -1.225558 \\
\hline $\mathrm{C}$ & 3.687682 & -2.441830 & -2.756634 \\
\hline $\mathrm{H}$ & 2.325872 & -3.342020 & -1.494471 \\
\hline $\mathrm{H}$ & 2.301343 & -3.737850 & 1.023792 \\
\hline $\mathrm{C}$ & 0.765278 & -5.237852 & 0.898688 \\
\hline $\mathrm{C}$ & -1.333936 & -4.451056 & 0.104692 \\
\hline $\mathrm{H}$ & -1.514826 & -2.417190 & -0.371009 \\
\hline $\mathrm{H}$ & 0.073203 & -1.269918 & 2.800571 \\
\hline $\mathrm{C}$ & 1.742036 & -1.090601 & 4.125398 \\
\hline $\mathrm{C}$ & 3.906797 & -0.948362 & 3.079294 \\
\hline $\mathrm{H}$ & 3.902082 & -0.936958 & 0.940829 \\
\hline $\mathrm{C}$ & 4.264263 & -1.270189 & -3.238492 \\
\hline $\mathrm{H}$ & 4.285763 & 0.861930 & -3.147688 \\
\hline $\mathrm{C}$ & 3.346511 & 2.218267 & -0.406853 \\
\hline $\mathrm{C}$ & 1.417951 & 1.905130 & -1.786257 \\
\hline $\mathrm{H}$ & 3.970466 & -3.391755 & -3.192878 \\
\hline $\mathrm{C}$ & -0.512827 & -5.488484 & 0.573621 \\
\hline $\mathrm{H}$ & 1.400454 & -6.032393 & 1.268872 \\
\hline $\mathrm{H}$ & -2.365589 & -4.660526 & -0.144574 \\
\hline $\mathrm{C}$ & 3.129976 & -0.996591 & 4.236689 \\
\hline $\mathrm{H}$ & 1.130255 & -1.155692 & 5.015826 \\
\hline $\mathrm{H}$ & 4.985553 & -0.906391 & 3.151955 \\
\hline $\mathrm{H}$ & 4.983717 & -1.312481 & -4.046062 \\
\hline $\mathrm{C}$ & 2.948272 & 3.551113 & -0.192154 \\
\hline $\mathrm{N}$ & 4.553751 & 1.741193 & 0.218337 \\
\hline $\mathrm{C}$ & 1.038636 & 3.235259 & -1.558209 \\
\hline $\mathrm{H}$ & 0.819927 & 1.316119 & -2.464334 \\
\hline $\mathrm{H}$ & -0.913513 & -6.488512 & 0.679698 \\
\hline $\mathrm{H}$ & 3.600122 & -0.981065 & 5.211315 \\
\hline $\mathrm{C}$ & 1.781124 & 4.036299 & -0.783415 \\
\hline
\end{tabular}




\begin{tabular}{lrrr}
$\mathrm{H}$ & 3.544707 & 4.214226 & 0.420705 \\
$\mathrm{C}$ & 4.744338 & 1.976618 & 1.650840 \\
$\mathrm{C}$ & 5.751627 & 1.515764 & -0.591804 \\
$\mathrm{H}$ & 0.150845 & 3.627338 & -2.032697 \\
$\mathrm{H}$ & 1.480891 & 5.063695 & -0.621312 \\
$\mathrm{H}$ & 5.466179 & 1.242637 & 2.064810 \\
$\mathrm{H}$ & 5.148109 & 2.997653 & 1.815538 \\
$\mathrm{H}$ & 3.779195 & 1.877389 & 2.192651 \\
$\mathrm{H}$ & 6.622667 & 2.049758 & -0.154702 \\
$\mathrm{H}$ & 5.976341 & 0.429454 & -0.626621 \\
$\mathrm{H}$ & 5.617951 & 1.906438 & -1.620699 \\
$\mathrm{H}$ & -2.190335 & 1.191013 & -2.877518 \\
$\mathrm{H}$ & -2.234107 & 3.687491 & -2.699791 \\
$\mathrm{Cl}$ & -3.170783 & 5.399428 & -0.601848 \\
\hline \hline
\end{tabular}

Table S43: Cartesian coordinates $(\AA)$ of t2oTS $(\mathrm{E}=$ 1748.317306219510 a.u.).

\begin{tabular}{lrrr}
\hline \hline $\mathrm{C}$ & 0.155276 & -2.569804 & 3.031764 \\
$\mathrm{C}$ & 0.159129 & -1.889424 & 1.809659 \\
$\mathrm{C}$ & 0.930956 & -0.738197 & 1.603038 \\
$\mathrm{C}$ & 1.732783 & -0.274079 & 2.664343 \\
$\mathrm{C}$ & 1.714800 & -0.955922 & 3.890984 \\
$\mathrm{C}$ & 0.933051 & -2.095251 & 4.086053 \\
$\mathrm{Pd}$ & 0.800635 & 0.271477 & -0.173375 \\
$\mathrm{C}$ & 2.666577 & 0.892297 & 2.465890 \\
$\mathrm{C}$ & 3.998043 & 0.449657 & 1.815806 \\
$\mathrm{C}$ & 3.859479 & -0.437318 & 0.596540 \\
$\mathrm{C}$ & 2.990719 & -0.077649 & -0.473768 \\
$\mathrm{C}$ & 3.030426 & -0.874525 & -1.641346 \\
$\mathrm{C}$ & 3.838087 & -2.008783 & -1.752704 \\
$\mathrm{C}$ & 4.640411 & -2.342634 & -0.667274 \\
$\mathrm{C}$ & 4.673080 & -1.569653 & 0.493021 \\
$\mathrm{H}$ & -0.483414 & -2.251789 & 1.019478 \\
$\mathrm{H}$ & 5.335639 & -1.849413 & 1.307065 \\
$\mathrm{H}$ & 2.331485 & -0.579290 & 4.706592 \\
$\mathrm{H}$ & 0.931787 & -2.601763 & 5.049065 \\
$\mathrm{H}$ & -0.465470 & -3.455813 & 3.153803 \\
$\mathrm{H}$ & 3.027997 & 1.241012 & -0.687882 \\
$\mathrm{H}$ & 2.899891 & 1.372968 & 3.424484 \\
$\mathrm{H}$ & 2.213938 & 1.660311 & 1.835596 \\
$\mathrm{H}$ & 4.618051 & -0.065165 & 2.561871 \\
$\mathrm{H}$ & 4.514896 & 1.372259 & 1.518402 \\
$\mathrm{Br}$ & 0.389108 & 1.539113 & -2.539598 \\
$\mathrm{O}$ & 3.411767 & 2.406070 & -1.112357 \\
$\mathrm{C}$ & 3.365783 & 3.432322 & -0.322852 \\
$\mathrm{O}$ & 3.587493 & 3.505745 & 0.882120 \\
$\mathrm{O}$ & 3.023240 & 4.590174 & -0.997060 \\
$\mathrm{H}$ & 2.770838 & 4.279450 & -1.883754 \\
$\mathrm{P}$ & -1.462083 & 0.855490 & 0.141580 \\
$\mathrm{C}$ & -2.561373 & 0.516993 & -1.303646
\end{tabular}




\begin{tabular}{|c|c|c|c|}
\hline C & -1.487763 & 2.655786 & 0.451040 \\
\hline $\mathrm{C}$ & -2.351769 & 0.294166 & 1.642845 \\
\hline $\mathrm{C}$ & -2.668495 & -0.782606 & -1.885310 \\
\hline $\mathrm{C}$ & -3.282131 & 1.578665 & -1.896484 \\
\hline $\mathrm{C}$ & -2.666085 & 3.307341 & 0.868161 \\
\hline $\mathrm{C}$ & -0.335522 & 3.355056 & 0.379644 \\
\hline $\mathrm{C}$ & -1.789456 & 0.503753 & 2.852474 \\
\hline $\mathrm{C}$ & -3.662018 & -0.213331 & 1.563317 \\
\hline $\mathrm{C}$ & -3.459112 & -0.943157 & -3.035548 \\
\hline $\mathrm{C}$ & -1.845853 & -1.949000 & -1.471369 \\
\hline $\mathrm{C}$ & -4.067550 & 1.386887 & -2.966318 \\
\hline $\mathrm{H}$ & -3.205730 & 2.584396 & -1.522877 \\
\hline $\mathrm{H}$ & -3.596653 & 2.763182 & 0.966244 \\
\hline $\mathrm{C}$ & -2.632360 & 4.677798 & 1.151315 \\
\hline $\mathrm{C}$ & -0.305276 & 4.673718 & 0.656296 \\
\hline $\mathrm{H}$ & 0.584541 & 2.856254 & 0.108140 \\
\hline $\mathrm{H}$ & -0.811096 & 0.958738 & 2.927525 \\
\hline $\mathrm{C}$ & -2.462490 & 0.166430 & 4.032933 \\
\hline $\mathrm{C}$ & -4.366080 & -0.528746 & 2.730644 \\
\hline $\mathrm{H}$ & -4.154551 & -0.342627 & 0.610644 \\
\hline $\mathrm{C}$ & -4.175233 & 0.130396 & -3.556012 \\
\hline $\mathrm{H}$ & -3.493716 & -1.892444 & -3.554124 \\
\hline $\mathrm{C}$ & -2.396416 & -3.066007 & -0.780030 \\
\hline $\mathrm{C}$ & -0.571585 & -2.019938 & -1.917838 \\
\hline $\mathrm{H}$ & -4.606051 & 2.223001 & -3.394761 \\
\hline $\mathrm{C}$ & -1.473991 & 5.348693 & 1.043468 \\
\hline $\mathrm{H}$ & -3.533964 & 5.191185 & 1.460013 \\
\hline $\mathrm{H}$ & 0.628852 & 5.216656 & 0.589319 \\
\hline $\mathrm{C}$ & -3.756336 & -0.353750 & 3.972938 \\
\hline $\mathrm{H}$ & -1.987952 & 0.329172 & 4.991938 \\
\hline $\mathrm{H}$ & -5.378640 & -0.905794 & 2.671562 \\
\hline $\mathrm{H}$ & -4.788397 & -0.003600 & -4.437849 \\
\hline $\mathrm{C}$ & -1.585421 & -4.196426 & -0.566399 \\
\hline $\mathrm{N}$ & -3.746428 & -3.068969 & -0.277223 \\
\hline $\mathrm{C}$ & 0.221073 & -3.153818 & -1.691143 \\
\hline $\mathrm{H}$ & -0.152281 & -1.214645 & -2.500920 \\
\hline $\mathrm{H}$ & -1.438021 & 6.407865 & 1.264215 \\
\hline $\mathrm{H}$ & -4.287868 & -0.602460 & 4.882213 \\
\hline $\mathrm{C}$ & -0.270554 & -4.212470 & -1.034410 \\
\hline $\mathrm{H}$ & -1.972253 & -5.063861 & -0.047804 \\
\hline $\mathrm{C}$ & -3.977294 & -3.450204 & 1.117365 \\
\hline $\mathrm{C}$ & -4.867874 & -3.204797 & -1.207626 \\
\hline $\mathrm{H}$ & 1.233148 & -3.184099 & -2.070103 \\
\hline $\mathrm{H}$ & 0.349327 & -5.084976 & -0.871986 \\
\hline $\mathrm{H}$ & -4.942743 & -3.033360 & 1.471553 \\
\hline $\mathrm{H}$ & -4.018572 & -4.556228 & 1.204782 \\
\hline $\mathrm{H}$ & -3.160293 & -3.066163 & 1.765378 \\
\hline $\mathrm{H}$ & -5.534987 & -4.035894 & -0.892807 \\
\hline $\mathrm{H}$ & -5.451760 & -2.261086 & -1.224369 \\
\hline $\mathrm{H}$ & -4.512621 & -3.446008 & -2.229802 \\
\hline $\mathrm{H}$ & 2.421039 & -0.576233 & -2.490489 \\
\hline
\end{tabular}




\begin{tabular}{llll}
$\mathrm{H}$ & 3.847959 & -2.613860 & -2.653850 \\
$\mathrm{Cl}$ & 5.673299 & -3.780415 & -0.766285 \\
\hline \hline
\end{tabular}

Table S44: Cartesian coordinates $(\AA)$ of t2pR (E= 1748.327312599973 a.u.).

\begin{tabular}{lrrr}
\hline \hline $\mathrm{C}$ & -0.090216 & -3.095784 & 2.420810 \\
$\mathrm{C}$ & -0.141366 & -2.257417 & 1.303135 \\
$\mathrm{C}$ & 0.853671 & -1.305060 & 1.054948 \\
$\mathrm{C}$ & 1.928271 & -1.176595 & 1.958436 \\
$\mathrm{C}$ & 1.957782 & -2.020454 & 3.081721 \\
$\mathrm{C}$ & 0.965658 & -2.970627 & 3.321419 \\
$\mathrm{Pd}$ & 0.679532 & -0.088525 & -0.579581 \\
$\mathrm{C}$ & 3.063114 & -0.207929 & 1.725846 \\
$\mathrm{C}$ & 4.176959 & -0.806264 & 0.815237 \\
$\mathrm{C}$ & 3.599999 & -1.483802 & -0.394939 \\
$\mathrm{C}$ & 2.949437 & -0.672093 & -1.367509 \\
$\mathrm{C}$ & 2.358399 & -1.243130 & -2.509063 \\
$\mathrm{C}$ & 2.402132 & -2.629289 & -2.705249 \\
$\mathrm{C}$ & 3.040084 & -3.434213 & -1.770634 \\
$\mathrm{C}$ & 3.623265 & -2.858606 & -0.634480 \\
$\mathrm{H}$ & -0.988565 & -2.336793 & 0.636938 \\
$\mathrm{H}$ & 2.785504 & -1.918174 & 3.781564 \\
$\mathrm{H}$ & 1.016168 & -3.601644 & 4.206070 \\
$\mathrm{H}$ & -0.881046 & -3.825114 & 2.585527 \\
$\mathrm{H}$ & 3.131798 & 0.408299 & -1.293726 \\
$\mathrm{H}$ & 3.526470 & 0.082715 & 2.674354 \\
$\mathrm{H}$ & 2.728204 & 0.728375 & 1.273059 \\
$\mathrm{H}$ & 4.790626 & -1.506904 & 1.387158 \\
$\mathrm{H}$ & 4.781283 & 0.044518 & 0.473951 \\
$\mathrm{Br}$ & 0.295549 & 1.582563 & -2.659159 \\
$\mathrm{O}$ & 4.600052 & 1.839994 & -0.806469 \\
$\mathrm{C}$ & 4.559300 & 2.716102 & 0.108554 \\
$\mathrm{O}$ & 4.204519 & 2.636228 & 1.294579 \\
$\mathrm{O}$ & 5.006089 & 3.994718 & -0.306614 \\
$\mathrm{H}$ & 5.205676 & 3.845999 & -1.245361 \\
$\mathrm{P}$ & -1.089614 & 1.091308 & 0.331945 \\
$\mathrm{C}$ & -2.508448 & 1.313541 & -0.828481 \\
$\mathrm{C}$ & -0.472881 & 2.753047 & 0.775167 \\
$\mathrm{C}$ & -1.789197 & 0.585545 & 1.948744 \\
$\mathrm{C}$ & -3.143405 & 0.209715 & -1.475835 \\
$\mathrm{C}$ & -2.958327 & 2.618593 & -1.133878 \\
$\mathrm{C}$ & -1.264524 & 3.635929 & 1.538099 \\
$\mathrm{C}$ & 0.784395 & 3.114261 & 0.440869 \\
$\mathrm{C}$ & -0.963803 & 0.469370 & 3.011230 \\
$\mathrm{C}$ & -3.179381 & 0.467440 & 2.133774 \\
-4.159849 & 0.470999 & -2.410297 \\
$\mathrm{H}$ & -2.676214 & -1.197932 & -1.377301 \\
$\mathrm{H}$ & -2.492276 & 2.835318 & -1.993613 \\
$\mathrm{H}$ & 3.486750 & -0.702448 \\
$\mathrm{H}$ & & & \\
$\mathrm{H}$ & &
\end{tabular}




\begin{tabular}{rrrr}
$\mathrm{C}$ & -0.753035 & 4.888496 & 1.895361 \\
$\mathrm{C}$ & 1.272463 & 4.323244 & 0.784595 \\
$\mathrm{H}$ & 1.419814 & 2.431019 & -0.106499 \\
$\mathrm{H}$ & 0.097873 & 0.638105 & 2.897603 \\
$\mathrm{C}$ & -1.458739 & 0.165228 & 4.285044 \\
$\mathrm{C}$ & -3.699896 & 0.195585 & 3.403892 \\
$\mathrm{H}$ & -3.866830 & 0.612187 & 1.313548 \\
$\mathrm{C}$ & -4.584916 & 1.774530 & -2.646763 \\
$\mathrm{H}$ & -4.601536 & -0.332551 & -2.985312 \\
$\mathrm{C}$ & -3.408428 & -2.200016 & -0.676863 \\
$\mathrm{C}$ & -1.618700 & -1.570612 & -2.133115 \\
$\mathrm{H}$ & -4.282976 & 3.848675 & -2.203566 \\
$\mathrm{C}$ & 0.490898 & 5.227035 & 1.521250 \\
$\mathrm{H}$ & -1.357143 & 5.578323 & 2.470621 \\
$\mathrm{H}$ & 2.277390 & 4.601368 & 0.496392 \\
$\mathrm{C}$ & -2.833057 & 0.025176 & 4.483090 \\
$\mathrm{H}$ & -0.777872 & 0.062690 & 5.120069 \\
$\mathrm{H}$ & -4.769326 & 0.120425 & 3.550419 \\
$\mathrm{H}$ & -5.374828 & 1.965941 & -3.361577 \\
$\mathrm{C}$ & -2.990226 & -3.540188 & -0.780212 \\
$\mathrm{~N}$ & -4.549943 & -1.886360 & 0.144485 \\
$\mathrm{C}$ & -1.216958 & -2.910663 & -2.219520 \\
$\mathrm{H}$ & -1.088810 & -0.843194 & -2.728165 \\
$\mathrm{H}$ & 0.894909 & 6.193851 & 1.793096 \\
$\mathrm{H}$ & -3.223674 & -0.193681 & 5.468322 \\
$\mathrm{C}$ & -1.882634 & -3.869089 & -1.562709 \\
$\mathrm{H}$ & -3.525281 & -4.327597 & -0.265789 \\
$\mathrm{C}$ & -4.603831 & -2.420399 & 1.506888 \\
$\mathrm{C}$ & -5.818811 & -1.517836 & -0.484910 \\
$\mathrm{H}$ & -0.376400 & -3.177691 & -2.843626 \\
$\mathrm{H}$ & -1.566956 & -4.901364 & -1.644211 \\
$\mathrm{H}$ & -5.292861 & -1.811982 & 2.128315 \\
$\mathrm{H}$ & -4.978617 & -3.465278 & 1.488881 \\
$\mathrm{H}$ & -3.593675 & -2.405511 & 1.969824 \\
$\mathrm{H}$ & -6.645811 & -2.145713 & -0.088994 \\
$\mathrm{H}$ & -6.042550 & -0.451938 & -0.271407 \\
$\mathrm{H}$ & -5.784864 & -1.683989 & -1.580473 \\
$\mathrm{H}$ & 1.914473 & -0.590335 & -3.252266 \\
\hline & 1.964166 & -3.074418 & -3.594559 \\
$\mathrm{H} \mathrm{r}$ & 4.422794 & -3.974369 & 0.489918 \\
3.093104 & -4.510013 & -1.906280 \\
\hline & & & \\
\hline
\end{tabular}

Table S45: Cartesian coordinates $(\AA)$ of t2pTS $(\mathrm{E}=$ 1748.313360471957 a.u.).

\begin{tabular}{llll}
\hline \hline $\mathrm{C}$ & 0.251970 & -3.138517 & 2.389515 \\
$\mathrm{C}$ & 0.167113 & -2.249900 & 1.312549 \\
$\mathrm{C}$ & 1.015164 & -1.140411 & 1.202792 \\
$\mathrm{C}$ & 1.982033 & -0.931569 & 2.204711 \\
$\mathrm{C}$ & 2.053551 & -1.822612 & 3.286400 \\
$\mathrm{C}$ & 1.197481 & -2.919826 & 3.389636
\end{tabular}




\begin{tabular}{|c|c|c|c|}
\hline $\mathrm{Pd}$ & 0.782505 & 0.186350 & -0.333405 \\
\hline $\mathrm{C}$ & 2.983609 & 0.188418 & 2.078525 \\
\hline $\mathrm{C}$ & 4.194848 & -0.224574 & 1.207305 \\
\hline $\mathrm{C}$ & 3.842390 & -0.901846 & -0.098852 \\
\hline $\mathrm{C}$ & 2.890218 & -0.295177 & -0.975706 \\
\hline $\mathrm{C}$ & 2.677409 & -0.858037 & -2.252047 \\
\hline $\mathrm{C}$ & 3.328617 & -2.024453 & -2.658712 \\
\hline $\mathrm{C}$ & 4.232024 & -2.634027 & -1.794998 \\
\hline $\mathrm{C}$ & 4.485956 & -2.059376 & -0.545327 \\
\hline $\mathrm{H}$ & -0.600551 & -2.414679 & 0.568995 \\
\hline $\mathrm{H}$ & 2.800638 & -1.647221 & 4.059662 \\
\hline $\mathrm{H}$ & 1.269377 & -3.592666 & 4.241649 \\
\hline $\mathrm{H}$ & -0.429693 & -3.985587 & 2.445031 \\
\hline $\mathrm{H}$ & 3.038872 & 1.027054 & -0.961473 \\
\hline $\mathrm{H}$ & 3.365312 & 0.483071 & 3.064091 \\
\hline $\mathrm{H}$ & 2.525589 & 1.079490 & 1.645118 \\
\hline $\mathrm{H}$ & 4.853754 & -0.871882 & 1.791032 \\
\hline $\mathrm{H}$ & 4.739074 & 0.701560 & 0.976362 \\
\hline $\mathrm{Br}$ & 0.237380 & 1.915192 & -2.357306 \\
\hline $\mathrm{O}$ & 3.507360 & 2.206467 & -1.236189 \\
\hline $\mathrm{C}$ & 3.562204 & 3.109423 & -0.306332 \\
\hline $\mathrm{O}$ & 3.953158 & 3.011743 & 0.852827 \\
\hline $\mathrm{O}$ & 3.114628 & 4.339991 & -0.747974 \\
\hline $\mathrm{H}$ & 2.711799 & 4.145303 & -1.612518 \\
\hline $\mathrm{P}$ & -1.352894 & 0.883059 & 0.384814 \\
\hline $\mathrm{C}$ & -2.642541 & 0.916227 & -0.936898 \\
\hline $\mathrm{C}$ & -1.160163 & 2.587380 & 1.013547 \\
\hline $\mathrm{C}$ & -2.115395 & 0.118574 & 1.866377 \\
\hline $\mathrm{C}$ & -2.957499 & -0.237984 & -1.715814 \\
\hline $\mathrm{C}$ & -3.306146 & 2.128257 & -1.235069 \\
\hline $\mathrm{C}$ & -2.211409 & 3.237488 & 1.691348 \\
\hline $\mathrm{C}$ & 0.040662 & 3.195237 & 0.918365 \\
\hline $\mathrm{C}$ & -1.397549 & 0.047875 & 3.007920 \\
\hline $\mathrm{C}$ & -3.472871 & -0.252855 & 1.874528 \\
\hline $\mathrm{C}$ & -3.888053 & -0.112493 & -2.760940 \\
\hline $\mathrm{C}$ & -2.222683 & -1.526371 & -1.625698 \\
\hline $\mathrm{C}$ & -4.226052 & 2.207729 & -2.207514 \\
\hline $\mathrm{H}$ & -3.076880 & 3.036721 & -0.706655 \\
\hline $\mathrm{H}$ & -3.175230 & 2.759393 & 1.810175 \\
\hline $\mathrm{C}$ & -2.007073 & 4.521536 & 2.209876 \\
\hline $\mathrm{C}$ & 0.235899 & 4.430007 & 1.422116 \\
\hline $\mathrm{H}$ & 0.867099 & 2.686723 & 0.442367 \\
\hline $\mathrm{H}$ & -0.374857 & 0.398827 & 3.031583 \\
\hline $\mathrm{C}$ & -1.960986 & -0.449730 & 4.189077 \\
\hline $\mathrm{C}$ & -4.066400 & -0.723069 & 3.051173 \\
\hline $\mathrm{H}$ & -4.084200 & -0.156333 & 0.989216 \\
\hline $\mathrm{C}$ & -4.537889 & 1.097035 & -2.986746 \\
\hline $\mathrm{H}$ & -4.085022 & -0.941685 & -3.427926 \\
\hline $\mathrm{C}$ & -2.805654 & -2.702873 & -1.073294 \\
\hline $\mathrm{C}$ & -1.024068 & -1.619019 & -2.244285 \\
\hline $\mathrm{H}$ & -4.715374 & 3.152544 & -2.408824 \\
\hline
\end{tabular}




\begin{tabular}{lrrr}
$\mathrm{C}$ & -0.806508 & 5.107781 & 2.074277 \\
$\mathrm{H}$ & -2.811024 & 5.035410 & 2.721089 \\
$\mathrm{H}$ & 1.206375 & 4.901046 & 1.332804 \\
$\mathrm{C}$ & -3.301236 & -0.838716 & 4.211659 \\
$\mathrm{H}$ & -1.363748 & -0.511386 & 5.089559 \\
$\mathrm{H}$ & -5.113760 & -0.994772 & 3.062643 \\
$\mathrm{H}$ & -5.259376 & 1.183211 & -3.788988 \\
$\mathrm{C}$ & -2.101245 & -3.917422 & -1.172311 \\
$\mathrm{~N}$ & -4.081296 & -2.687228 & -0.403762 \\
$\mathrm{C}$ & -0.337887 & -2.838452 & -2.330379 \\
$\mathrm{H}$ & -0.591026 & -0.755895 & -2.726489 \\
$\mathrm{H}$ & -0.638392 & 6.098975 & 2.475703 \\
$\mathrm{H}$ & -3.747234 & -1.209337 & 5.125380 \\
$\mathrm{C}$ & -0.860165 & -3.956275 & -1.809525 \\
$\mathrm{H}$ & -2.515735 & -4.831250 & -0.767191 \\
$\mathrm{C}$ & -4.190589 & -3.308773 & 0.917388 \\
$\mathrm{C}$ & -5.310463 & -2.545993 & -1.185463 \\
$\mathrm{H}$ & 0.612636 & -2.882870 & -2.843014 \\
$\mathrm{H}$ & -0.323488 & -4.893079 & -1.888738 \\
$\mathrm{H}$ & -5.057827 & -2.887110 & 1.466357 \\
$\mathrm{H}$ & -4.341991 & -4.403255 & 0.808654 \\
$\mathrm{H}$ & -3.267597 & -3.128016 & 1.509289 \\
$\mathrm{H}$ & -6.025655 & -3.358799 & -0.934984 \\
$\mathrm{H}$ & -5.783505 & -1.567947 & -0.958649 \\
$\mathrm{H}$ & -5.104238 & -2.622019 & -2.272137 \\
$\mathrm{H}$ & 2.005042 & -0.346341 & -2.934987 \\
$\mathrm{H}$ & 3.145146 & -2.453903 & -3.641314 \\
$\mathrm{Cl}$ & 5.715293 & -2.892675 & 0.448685 \\
$\mathrm{H}$ & 4.759768 & -3.538233 & -2.082410 \\
\hline \hline & & &
\end{tabular}

Table S46: Cartesian coordinates $(\AA)$ of t2qR $(E=-$ 1697.400685030663 a.u.).

\begin{tabular}{lrrr}
\hline \hline $\mathrm{C}$ & -1.815091 & -0.346460 & 3.947902 \\
$\mathrm{C}$ & -1.282616 & -0.527494 & 2.666742 \\
$\mathrm{C}$ & -0.200802 & 0.235041 & 2.215454 \\
$\mathrm{C}$ & 0.361243 & 1.211499 & 3.061197 \\
$\mathrm{C}$ & -0.191257 & 1.388649 & 4.339851 \\
$\mathrm{C}$ & -1.271121 & 0.624912 & 4.786555 \\
$\mathrm{Pd}$ & 0.538330 & 0.004412 & 0.327920 \\
$\mathrm{C}$ & 1.593832 & 1.971036 & 2.647179 \\
$\mathrm{C}$ & 2.874789 & 1.117896 & 2.861499 \\
$\mathrm{C}$ & 2.771074 & -0.302064 & 2.356288 \\
$\mathrm{C}$ & 3.065467 & -0.603970 & 1.004648 \\
$\mathrm{C}$ & 3.200815 & -1.951323 & 0.567574 \\
$\mathrm{C}$ & 2.909240 & -2.968786 & 1.487560 \\
$\mathrm{C}$ & 2.526254 & -2.677281 & 2.801501 \\
$\mathrm{C}$ & 2.478322 & -1.358483 & 3.237346 \\
$\mathrm{H}$ & -1.736419 & -1.261432 & 2.015224 \\
$\mathrm{H}$ & 2.235910 & -1.133552 & 4.273350 \\
$\mathrm{H}$ & 0.242153 & 2.145565 & 4.992056
\end{tabular}




\begin{tabular}{|c|c|c|c|}
\hline $\mathrm{H}$ & -1.684942 & 0.792188 & 5.778742 \\
\hline $\mathrm{H}$ & -2.657214 & -0.954235 & 4.273392 \\
\hline $\mathrm{H}$ & 3.422034 & 0.222403 & 0.385553 \\
\hline $\mathrm{H}$ & 1.697095 & 2.898837 & 3.221130 \\
\hline $\mathrm{H}$ & 1.575243 & 2.273417 & 1.596477 \\
\hline $\mathrm{H}$ & 3.099679 & 1.084256 & 3.936436 \\
\hline $\mathrm{H}$ & 3.687010 & 1.629069 & 2.332573 \\
\hline $\mathrm{Br}$ & 1.247201 & -0.139759 & -2.257263 \\
\hline $\mathrm{O}$ & 4.724258 & 1.851132 & 0.170788 \\
\hline $\mathrm{C}$ & 4.203122 & 2.993752 & 0.004423 \\
\hline $\mathrm{O}$ & 3.362289 & 3.604180 & 0.684642 \\
\hline $\mathrm{O}$ & 4.672395 & 3.685716 & -1.137483 \\
\hline $\mathrm{H}$ & 5.283696 & 3.043789 & -1.533557 \\
\hline $\mathrm{P}$ & -1.337109 & 0.966445 & -0.610375 \\
\hline $\mathrm{C}$ & -2.036469 & 0.000332 & -2.019888 \\
\hline $\mathrm{C}$ & -0.862653 & 2.617431 & -1.236552 \\
\hline $\mathrm{C}$ & -2.748397 & 1.445566 & 0.459110 \\
\hline $\mathrm{C}$ & -2.357063 & -1.387146 & -1.907528 \\
\hline C & -2.213628 & 0.629587 & -3.273196 \\
\hline $\mathrm{C}$ & -1.841689 & 3.528266 & -1.683957 \\
\hline $\mathrm{C}$ & 0.427839 & 3.011360 & -1.180855 \\
\hline $\mathrm{C}$ & -2.531554 & 2.289397 & 1.491251 \\
\hline $\mathrm{C}$ & -4.065816 & 1.071123 & 0.134750 \\
\hline $\mathrm{C}$ & -2.798091 & -2.071451 & -3.052749 \\
\hline $\mathrm{C}$ & -2.077813 & -2.214100 & -0.704609 \\
\hline $\mathrm{C}$ & -2.682782 & -0.037248 & -4.337664 \\
\hline $\mathrm{H}$ & -1.955214 & 1.662809 & -3.421931 \\
\hline $\mathrm{H}$ & -2.887856 & 3.251232 & -1.708820 \\
\hline $\mathrm{C}$ & -1.455048 & 4.804202 & -2.109579 \\
\hline $\mathrm{C}$ & 0.796683 & 4.241398 & -1.590929 \\
\hline $\mathrm{H}$ & 1.185151 & 2.341770 & -0.797600 \\
\hline $\mathrm{H}$ & -1.533447 & 2.647451 & 1.701921 \\
\hline C & -3.586947 & 2.746563 & 2.289506 \\
\hline C & -5.139768 & 1.539239 & 0.899960 \\
\hline $\mathrm{H}$ & -4.274764 & 0.443811 & -0.718956 \\
\hline $\mathrm{C}$ & -2.984284 & -1.393533 & -4.253383 \\
\hline $\mathrm{H}$ & -2.965345 & -3.140493 & -3.027362 \\
\hline $\mathrm{C}$ & -3.112926 & -2.703307 & 0.143486 \\
\hline $\mathrm{C}$ & -0.816692 & -2.666454 & -0.523000 \\
\hline $\mathrm{H}$ & -2.805942 & 0.476925 & -5.282699 \\
\hline $\mathrm{C}$ & -0.159167 & 5.152190 & -2.067434 \\
\hline $\mathrm{H}$ & -2.197605 & 5.506269 & -2.466594 \\
\hline $\mathrm{H}$ & 1.837375 & 4.534999 & -1.546660 \\
\hline $\mathrm{C}$ & -4.897503 & 2.368188 & 1.995021 \\
\hline $\mathrm{H}$ & -3.388638 & 3.411081 & 3.120445 \\
\hline $\mathrm{H}$ & -6.153983 & 1.263452 & 0.642745 \\
\hline $\mathrm{H}$ & -3.332469 & -1.926714 & -5.128634 \\
\hline C & -2.779517 & -3.619712 & 1.158644 \\
\hline $\mathrm{N}$ & -4.483770 & -2.282236 & 0.005001 \\
\hline $\mathrm{C}$ & -0.505961 & -3.578580 & 0.493950 \\
\hline $\mathrm{H}$ & -0.027579 & -2.371024 & -1.196101 \\
\hline
\end{tabular}




\begin{tabular}{lrrr}
$\mathrm{H}$ & 0.148757 & 6.137789 & -2.392431 \\
$\mathrm{H}$ & -5.719562 & 2.730317 & 2.598555 \\
$\mathrm{C}$ & -1.458137 & -4.040906 & 1.314014 \\
$\mathrm{H}$ & -3.540650 & -4.014559 & 1.818732 \\
$\mathrm{C}$ & -5.201148 & -1.818183 & 1.194133 \\
$\mathrm{C}$ & -5.285326 & -2.775847 & -1.115655 \\
$\mathrm{H}$ & 0.507878 & -3.936151 & 0.598302 \\
$\mathrm{H}$ & -1.206026 & -4.751729 & 2.090585 \\
$\mathrm{H}$ & -6.061453 & -1.183360 & 0.897250 \\
$\mathrm{H}$ & -5.587638 & -2.687814 & 1.765905 \\
$\mathrm{H}$ & -4.526106 & -1.225341 & 1.848002 \\
$\mathrm{H}$ & -6.235082 & -3.220294 & -0.747782 \\
$\mathrm{H}$ & -5.522682 & -1.935045 & -1.800138 \\
$\mathrm{H}$ & -4.750008 & -3.572932 & -1.669949 \\
$\mathrm{H}$ & 3.014693 & -4.012702 & 1.195412 \\
$\mathrm{H}$ & 2.302697 & -3.486821 & 3.494173 \\
$\mathrm{Si}$ & 4.097498 & -2.429300 & -1.046990 \\
$\mathrm{C}$ & 2.997217 & -3.542571 & -2.121239 \\
$\mathrm{H}$ & 2.603472 & -4.390149 & -1.544919 \\
$\mathrm{H}$ & 2.149729 & -2.973074 & -2.514261 \\
$\mathrm{H}$ & 3.563101 & -3.951281 & -2.969101 \\
$\mathrm{C}$ & 5.576720 & -3.497306 & -0.478414 \\
$\mathrm{H}$ & 6.154184 & -3.845083 & -1.345616 \\
$\mathrm{H}$ & 6.254479 & -2.918232 & 0.160643 \\
$\mathrm{H}$ & 5.262445 & -4.382772 & 0.087929 \\
$\mathrm{C}$ & 4.793564 & -0.947146 & -1.986827 \\
$\mathrm{H}$ & 4.087880 & -0.599697 & -2.746277 \\
$\mathrm{H}$ & 4.993205 & -0.093465 & -1.325282 \\
$\mathrm{H}$ & 5.732578 & -1.229561 & -2.482627 \\
\hline \hline & & &
\end{tabular}

Table S47: Cartesian coordinates $(\AA)$ of t2qTS $(\mathrm{E}=$ 1697.372983934153 a.u.).

\begin{tabular}{lrrr}
\hline \hline $\mathrm{C}$ & -1.012061 & 0.078842 & 4.177850 \\
$\mathrm{C}$ & -0.649128 & -0.210635 & 2.856658 \\
$\mathrm{C}$ & 0.348638 & 0.515577 & 2.189889 \\
$\mathrm{C}$ & 0.979646 & 1.567212 & 2.885259 \\
$\mathrm{C}$ & 0.600978 & 1.857333 & 4.203750 \\
$\mathrm{C}$ & -0.387980 & 1.121747 & 4.857927 \\
$\mathrm{Pd}$ & 0.812318 & 0.226221 & 0.207680 \\
$\mathrm{C}$ & 2.128880 & 2.303748 & 2.249543 \\
$\mathrm{C}$ & 3.431438 & 1.480996 & 2.380472 \\
$\mathrm{C}$ & 3.299178 & -0.009407 & 2.120107 \\
$\mathrm{C}$ & 2.890423 & -0.517830 & 0.849922 \\
$\mathrm{C}$ & 3.065585 & -1.928722 & 0.607075 \\
$\mathrm{C}$ & 3.426735 & -2.757089 & 1.690544 \\
$\mathrm{C}$ & 3.689706 & -2.246382 & 2.960503 \\
$\mathrm{C}$ & 3.682110 & -0.870980 & 3.155009 \\
$\mathrm{H}$ & -1.208109 & -0.986998 & 2.349502 \\
$\mathrm{H}$ & 3.965453 & -0.455524 & 4.120676 \\
$\mathrm{H}$ & 1.098055 & 2.676816 & 4.721937
\end{tabular}




\begin{tabular}{|c|c|c|c|}
\hline $\mathrm{H}$ & -0.670261 & 1.366488 & 5.879854 \\
\hline $\mathrm{H}$ & -1.795285 & -0.505207 & 4.658222 \\
\hline $\mathrm{H}$ & 3.342097 & 0.399512 & -0.151623 \\
\hline $\mathrm{H}$ & 2.281619 & 3.283025 & 2.720571 \\
\hline $\mathrm{H}$ & 1.933887 & 2.493136 & 1.192221 \\
\hline $\mathrm{H}$ & 3.823624 & 1.613934 & 3.397562 \\
\hline $\mathrm{H}$ & 4.156991 & 1.913444 & 1.680703 \\
\hline $\mathrm{Br}$ & 1.048169 & 0.121483 & -2.540914 \\
\hline $\mathrm{O}$ & 3.959051 & 1.005328 & -0.990861 \\
\hline C & 3.882663 & 2.310241 & -1.084267 \\
\hline $\mathrm{O}$ & 4.164279 & 3.149810 & -0.238964 \\
\hline $\mathrm{O}$ & 3.457130 & 2.725659 & -2.318619 \\
\hline $\mathrm{H}$ & 3.066716 & 1.932261 & -2.735314 \\
\hline $\mathrm{P}$ & -1.329348 & 1.053720 & -0.453813 \\
\hline $\mathrm{C}$ & -2.180889 & 0.030765 & -1.740059 \\
\hline $\mathrm{C}$ & -1.092510 & 2.724571 & -1.155473 \\
\hline $\mathrm{C}$ & -2.574818 & 1.460778 & 0.830814 \\
\hline $\mathrm{C}$ & -2.494516 & -1.351942 & -1.540924 \\
\hline $\mathrm{C}$ & -2.481618 & 0.612810 & -2.993451 \\
\hline $\mathrm{C}$ & -2.197584 & 3.536926 & -1.480694 \\
\hline $\mathrm{C}$ & 0.154203 & 3.224818 & -1.277494 \\
\hline C & -2.257533 & 2.357254 & 1.790352 \\
\hline C & -3.882447 & 0.949680 & 0.764928 \\
\hline $\mathrm{C}$ & -3.062320 & -2.069060 & -2.608235 \\
\hline $\mathrm{C}$ & -2.122036 & -2.129877 & -0.324213 \\
\hline $\mathrm{C}$ & -3.064922 & -0.087315 & -3.976095 \\
\hline $\mathrm{H}$ & -2.225305 & 1.634839 & -3.212237 \\
\hline $\mathrm{H}$ & -3.209215 & 3.169061 & -1.365556 \\
\hline $\mathrm{C}$ & -1.981571 & 4.831999 & -1.966133 \\
\hline $\mathrm{C}$ & 0.359720 & 4.472890 & -1.742391 \\
\hline $\mathrm{H}$ & 1.006104 & 2.625007 & -0.993679 \\
\hline $\mathrm{H}$ & -1.274379 & 2.807695 & 1.811594 \\
\hline C & -3.188685 & 2.738059 & 2.764191 \\
\hline $\mathrm{C}$ & -4.835948 & 1.327059 & 1.717172 \\
\hline $\mathrm{H}$ & -4.178272 & 0.285220 & -0.031329 \\
\hline $\mathrm{C}$ & -3.367558 & -1.434495 & -3.807824 \\
\hline $\mathrm{H}$ & -3.223133 & -3.134153 & -2.536035 \\
\hline C & -3.080935 & -2.870412 & 0.425821 \\
\hline $\mathrm{C}$ & -0.813925 & -2.273250 & -0.019373 \\
\hline $\mathrm{H}$ & -3.279116 & 0.391366 & -4.923544 \\
\hline $\mathrm{C}$ & -0.725789 & 5.289767 & -2.097277 \\
\hline $\mathrm{H}$ & -2.822335 & 5.460761 & -2.229776 \\
\hline $\mathrm{H}$ & 1.369132 & 4.852876 & -1.836159 \\
\hline $\mathrm{C}$ & -4.482822 & 2.216604 & 2.731837 \\
\hline $\mathrm{H}$ & -2.908715 & 3.446114 & 3.533359 \\
\hline $\mathrm{H}$ & -5.844025 & 0.937249 & 1.664531 \\
\hline $\mathrm{H}$ & -3.807139 & -1.996615 & -4.621734 \\
\hline $\mathrm{C}$ & -2.643001 & -3.689289 & 1.477850 \\
\hline $\mathrm{N}$ & -4.487447 & -2.829338 & 0.131584 \\
\hline $\mathrm{C}$ & -0.390913 & -3.118799 & 1.018260 \\
\hline $\mathrm{H}$ & -0.064156 & -1.759217 & -0.596849 \\
\hline
\end{tabular}




\begin{tabular}{lrrr}
$\mathrm{H}$ & -0.549464 & 6.291085 & -2.468928 \\
$\mathrm{H}$ & -5.210260 & 2.513263 & 3.476154 \\
$\mathrm{C}$ & -1.279892 & -3.801221 & 1.751795 \\
$\mathrm{H}$ & -3.356723 & -4.253712 & 2.064817 \\
$\mathrm{C}$ & -5.377430 & -2.073509 & 1.011329 \\
$\mathrm{C}$ & -5.079996 & -3.878433 & -0.699963 \\
$\mathrm{H}$ & 0.662484 & -3.220247 & 1.231988 \\
$\mathrm{H}$ & -0.941246 & -4.444668 & 2.553750 \\
$\mathrm{H}$ & -5.982845 & -1.365831 & 0.408006 \\
$\mathrm{H}$ & -6.059884 & -2.762801 & 1.552241 \\
$\mathrm{H}$ & -4.797834 & -1.510762 & 1.772834 \\
$\mathrm{H}$ & -5.803583 & -4.477500 & -0.107332 \\
$\mathrm{H}$ & -5.610145 & -3.416394 & -1.558747 \\
$\mathrm{H}$ & -4.302883 & -4.574858 & -1.081332 \\
$\mathrm{H}$ & 3.528915 & -3.828359 & 1.545448 \\
$\mathrm{H}$ & 3.947110 & -2.918023 & 3.778120 \\
$\mathrm{Si}$ & 3.244516 & -2.831495 & -1.091716 \\
$\mathrm{C}$ & 1.699672 & -3.505241 & -1.969615 \\
$\mathrm{H}$ & 1.080027 & -4.120717 & -1.305714 \\
$\mathrm{H}$ & 1.086954 & -2.709555 & -2.400020 \\
$\mathrm{H}$ & 2.037649 & -4.153263 & -2.790556 \\
$\mathrm{C}$ & 4.266228 & -4.421128 & -0.760103 \\
$\mathrm{H}$ & 4.538488 & -4.860394 & -1.729541 \\
$\mathrm{H}$ & 5.197085 & -4.220583 & -0.216706 \\
$\mathrm{H}$ & 3.711631 & -5.189045 & -0.205523 \\
$\mathrm{C}$ & 4.296699 & -1.830027 & -2.299412 \\
$\mathrm{H}$ & 4.369070 & -2.365429 & -3.256168 \\
$\mathrm{H}$ & 3.880053 & -0.837426 & -2.472727 \\
$\mathrm{H}$ & 5.312046 & -1.697589 & -1.905731 \\
\hline \hline & & &
\end{tabular}

Table S48: Cartesian coordinates $(\AA)$ of t2rR (E= 1697.413301520109 a.u.).

\begin{tabular}{lrrr}
\hline \hline $\mathrm{C}$ & 0.857401 & 0.065602 & 4.080782 \\
$\mathrm{C}$ & 0.620509 & 0.226302 & 2.712337 \\
$\mathrm{C}$ & -0.356843 & -0.520104 & 2.036874 \\
$\mathrm{C}$ & -1.100058 & -1.475977 & 2.759896 \\
$\mathrm{C}$ & -0.837239 & -1.635564 & 4.132106 \\
$\mathrm{C}$ & 0.125209 & -0.878512 & 4.797331 \\
$\mathrm{Pd}$ & -0.633951 & -0.280435 & 0.018120 \\
$\mathrm{C}$ & -2.232901 & -2.261185 & 2.145689 \\
$\mathrm{C}$ & -3.561484 & -1.462061 & 2.206483 \\
$\mathrm{C}$ & -3.463761 & -0.120070 & 1.540926 \\
$\mathrm{C}$ & -3.092708 & -0.033478 & 0.172376 \\
$\mathrm{C}$ & -3.078969 & 1.223225 & -0.467473 \\
$\mathrm{C}$ & -3.394879 & 2.418147 & 0.207832 \\
$\mathrm{C}$ & -3.722260 & 2.302266 & 1.570491 \\
$\mathrm{C}$ & -3.752614 & 1.066677 & 2.221993 \\
$\mathrm{H}$ & 1.255097 & 0.916780 & 2.171947 \\
$\mathrm{H}$ & -4.022115 & 1.021200 & 3.275210 \\
$\mathrm{H}$ & -1.413174 & -2.378272 & 4.682317
\end{tabular}




\begin{tabular}{|c|c|c|c|}
\hline $\mathrm{H}$ & 0.306662 & -1.032722 & 5.858909 \\
\hline $\mathrm{H}$ & 1.624726 & 0.664213 & 4.568011 \\
\hline $\mathrm{H}$ & -3.067003 & -0.968169 & -0.406993 \\
\hline $\mathrm{H}$ & -2.383414 & -3.202266 & 2.687958 \\
\hline $\mathrm{H}$ & -2.054445 & -2.526415 & 1.099130 \\
\hline $\mathrm{H}$ & -3.862111 & -1.327885 & 3.254774 \\
\hline $\mathrm{H}$ & -4.310634 & -2.093133 & 1.694479 \\
\hline $\mathrm{Br}$ & -0.753713 & -0.040526 & -2.697712 \\
\hline $\mathrm{O}$ & -3.385610 & -2.986577 & -0.766783 \\
\hline $\mathrm{C}$ & -4.314405 & -3.752585 & -0.381919 \\
\hline $\mathrm{O}$ & -5.043193 & -3.708104 & 0.624794 \\
\hline $\mathrm{O}$ & -4.553724 & -4.846662 & -1.247234 \\
\hline $\mathrm{H}$ & -3.906364 & -4.690836 & -1.954619 \\
\hline $\mathrm{P}$ & 1.481565 & -1.048775 & -0.488893 \\
\hline $\mathrm{C}$ & 2.386618 & 0.033195 & -1.680878 \\
\hline $\mathrm{C}$ & 1.284614 & -2.696314 & -1.250096 \\
\hline $\mathrm{C}$ & 2.639636 & -1.476695 & 0.864292 \\
\hline $\mathrm{C}$ & 2.569680 & 1.432640 & -1.452251 \\
\hline C & 2.846831 & -0.513210 & -2.900533 \\
\hline $\mathrm{C}$ & 2.402652 & -3.512077 & -1.515709 \\
\hline $\mathrm{C}$ & 0.044682 & -3.183711 & -1.464363 \\
\hline $\mathrm{C}$ & 2.251587 & -2.370882 & 1.799585 \\
\hline $\mathrm{C}$ & 3.957324 & -0.986347 & 0.874996 \\
\hline $\mathrm{C}$ & 3.155580 & 2.207746 & -2.466741 \\
\hline $\mathrm{C}$ & 2.051211 & 2.158814 & -0.260882 \\
\hline $\mathrm{C}$ & 3.449283 & 0.242885 & -3.829483 \\
\hline $\mathrm{H}$ & 2.704457 & -1.553026 & -3.136396 \\
\hline $\mathrm{H}$ & 3.406747 & -3.158109 & -1.319508 \\
\hline $\mathrm{C}$ & 2.210213 & -4.793833 & -2.044695 \\
\hline $\mathrm{C}$ & -0.138214 & -4.419181 & -1.970745 \\
\hline $\mathrm{H}$ & -0.820974 & -2.584554 & -1.220067 \\
\hline $\mathrm{H}$ & 1.258463 & -2.799447 & 1.765647 \\
\hline $\mathrm{C}$ & 3.120623 & -2.775416 & 2.819960 \\
\hline $\mathrm{C}$ & 4.851075 & -1.393284 & 1.872242 \\
\hline $\mathrm{H}$ & 4.306056 & -0.312036 & 0.106815 \\
\hline $\mathrm{C}$ & 3.613954 & 1.611608 & -3.637486 \\
\hline $\mathrm{H}$ & 3.224188 & 3.282246 & -2.371696 \\
\hline $\mathrm{C}$ & 2.919478 & 2.781819 & 0.680197 \\
\hline $\mathrm{C}$ & 0.718310 & 2.355605 & -0.144835 \\
\hline $\mathrm{H}$ & 3.787569 & -0.208720 & -4.753765 \\
\hline $\mathrm{C}$ & 0.962802 & -5.237087 & -2.271359 \\
\hline $\mathrm{H}$ & 3.062093 & -5.424601 & -2.264158 \\
\hline $\mathrm{H}$ & -1.141802 & -4.788792 & -2.139179 \\
\hline $\mathrm{C}$ & 4.425853 & -2.282636 & 2.859212 \\
\hline $\mathrm{H}$ & 2.785264 & -3.481560 & 3.568431 \\
\hline $\mathrm{H}$ & 5.868334 & -1.024944 & 1.877537 \\
\hline $\mathrm{H}$ & 4.071235 & 2.215971 & -4.410299 \\
\hline $\mathrm{C}$ & 2.363828 & 3.543233 & 1.721375 \\
\hline $\mathrm{N}$ & 4.351009 & 2.669614 & 0.597495 \\
\hline $\mathrm{C}$ & 0.181882 & 3.123819 & 0.900155 \\
\hline $\mathrm{H}$ & 0.039340 & 1.945772 & -0.875486 \\
\hline
\end{tabular}




\begin{tabular}{lrrr}
$\mathrm{H}$ & 0.804597 & -6.228306 & -2.676739 \\
$\mathrm{H}$ & 5.106304 & -2.599948 & 3.638628 \\
$\mathrm{C}$ & 0.980235 & 3.700453 & 1.808090 \\
$\mathrm{H}$ & 3.003705 & 4.022251 & 2.451712 \\
$\mathrm{C}$ & 5.076540 & 2.033868 & 1.696696 \\
$\mathrm{C}$ & 5.099543 & 3.580086 & -0.270064 \\
$\mathrm{H}$ & -0.886297 & 3.257494 & 0.974406 \\
$\mathrm{H}$ & 0.552896 & 4.291218 & 2.608242 \\
$\mathrm{H}$ & 5.879041 & 1.386282 & 1.287384 \\
$\mathrm{H}$ & 5.534824 & 2.808793 & 2.346958 \\
$\mathrm{H}$ & 4.392520 & 1.419662 & 2.320522 \\
$\mathrm{H}$ & 5.849454 & 4.148794 & 0.320192 \\
$\mathrm{H}$ & 5.626249 & 2.996832 & -1.053726 \\
$\mathrm{H}$ & 4.426812 & 4.323311 & -0.746613 \\
$\mathrm{H}$ & -3.964693 & 3.194684 & 2.146009 \\
$\mathrm{Si}$ & -3.447253 & 4.058387 & -0.722328 \\
$\mathrm{C}$ & -3.376743 & 5.504083 & 0.510417 \\
$\mathrm{H}$ & -4.235314 & 5.510742 & 1.192989 \\
$\mathrm{H}$ & -2.466426 & 5.461983 & 1.120908 \\
$\mathrm{H}$ & -3.374814 & 6.463913 & -0.021970 \\
$\mathrm{C}$ & -1.989974 & 4.210765 & -1.926078 \\
$\mathrm{H}$ & -1.066771 & 4.476517 & -1.397705 \\
$\mathrm{H}$ & -1.797535 & 3.273736 & -2.461279 \\
$\mathrm{H}$ & -2.180691 & 4.994052 & -2.671216 \\
$\mathrm{C}$ & -5.067557 & 4.180535 & -1.707555 \\
$\mathrm{H}$ & -5.135117 & 5.131366 & -2.252424 \\
$\mathrm{H}$ & -5.140138 & 3.368992 & -2.441473 \\
$\mathrm{H}$ & -5.941549 & 4.106048 & -1.049177 \\
$\mathrm{H}$ & -2.841608 & 1.238010 & -1.526037 \\
\hline \hline & & &
\end{tabular}

Table S49: Cartesian coordinates $(\AA)$ of t2rTS $(E=-$ 1697.394880546373 a.u.).

\begin{tabular}{lrrr}
\hline \hline $\mathrm{C}$ & -0.678934 & -0.973815 & 3.986263 \\
$\mathrm{C}$ & -0.406261 & -0.790070 & 2.626646 \\
$\mathrm{C}$ & 0.177423 & 0.386962 & 2.134623 \\
$\mathrm{C}$ & 0.492037 & 1.403768 & 3.058131 \\
$\mathrm{C}$ & 0.204461 & 1.216517 & 4.419238 \\
$\mathrm{C}$ & -0.376950 & 0.040099 & 4.892732 \\
$\mathrm{Pd}$ & 0.460038 & 0.637389 & 0.118711 \\
$\mathrm{C}$ & 1.205284 & 2.654721 & 2.613957 \\
$\mathrm{C}$ & 2.733273 & 2.440449 & 2.544507 \\
$\mathrm{C}$ & 3.189261 & 1.225620 & 1.766537 \\
$\mathrm{C}$ & 2.648755 & 0.929398 & 0.484330 \\
$\mathrm{C}$ & 3.253397 & -0.116197 & -0.254191 \\
$\mathrm{C}$ & 4.313706 & -0.904110 & 0.232069 \\
$\mathrm{C}$ & 4.779592 & -0.602646 & 1.524806 \\
$\mathrm{C}$ & 4.239234 & 0.447919 & 2.267441 \\
$\mathrm{H}$ & -0.704507 & -1.572743 & 1.942236 \\
$\mathrm{H}$ & 4.648122 & 0.672931 & 3.251882 \\
$\mathrm{H}$ & 0.445759 & 2.017558 & 5.117533
\end{tabular}




\begin{tabular}{|c|c|c|c|}
\hline $\mathrm{H}$ & -0.595555 & -0.077512 & 5.952235 \\
\hline $\mathrm{H}$ & -1.140651 & -1.900851 & 4.322287 \\
\hline $\mathrm{H}$ & 2.445006 & 2.057806 & -0.216576 \\
\hline $\mathrm{H}$ & 1.008746 & 3.481979 & 3.307757 \\
\hline $\mathrm{H}$ & 0.854972 & 2.982893 & 1.633509 \\
\hline $\mathrm{H}$ & 3.143828 & 2.386847 & 3.562220 \\
\hline $\mathrm{H}$ & 3.144908 & 3.335902 & 2.058345 \\
\hline $\mathrm{Br}$ & 0.603663 & 0.832352 & -2.603084 \\
\hline $\mathrm{O}$ & 2.645719 & 3.079512 & -0.986686 \\
\hline $\mathrm{C}$ & 2.224148 & 4.262260 & -0.669729 \\
\hline $\mathrm{O}$ & 2.111248 & 4.790392 & 0.432029 \\
\hline $\mathrm{O}$ & 1.874407 & 4.999671 & -1.787962 \\
\hline $\mathrm{H}$ & 1.933453 & 4.359356 & -2.518107 \\
\hline $\mathrm{P}$ & -1.840336 & 0.521265 & -0.379594 \\
\hline $\mathrm{C}$ & -2.273154 & -0.634233 & -1.754565 \\
\hline $\mathrm{C}$ & -2.347565 & 2.202720 & -0.881596 \\
\hline $\mathrm{C}$ & -3.050569 & 0.246786 & 0.969275 \\
\hline $\mathrm{C}$ & -1.896072 & -2.011736 & -1.733001 \\
\hline C & -2.936052 & -0.137941 & -2.900122 \\
\hline $\mathrm{C}$ & -3.705537 & 2.526231 & -1.075967 \\
\hline $\mathrm{C}$ & -1.416835 & 3.176830 & -0.966085 \\
\hline C & -2.999570 & 1.037853 & 2.062618 \\
\hline $\mathrm{C}$ & -4.103931 & -0.674603 & 0.824956 \\
\hline $\mathrm{C}$ & -2.167245 & -2.801763 & -2.862394 \\
\hline $\mathrm{C}$ & -1.075821 & -2.631740 & -0.659139 \\
\hline $\mathrm{C}$ & -3.226976 & -0.927789 & -3.943925 \\
\hline $\mathrm{H}$ & -3.206840 & 0.899646 & -2.984483 \\
\hline $\mathrm{H}$ & -4.475282 & 1.771175 & -0.980127 \\
\hline $\mathrm{C}$ & -4.060652 & 3.839766 & -1.404560 \\
\hline $\mathrm{C}$ & -1.762164 & 4.441393 & -1.279100 \\
\hline $\mathrm{H}$ & -0.377219 & 2.951684 & -0.770498 \\
\hline $\mathrm{H}$ & -2.229539 & 1.792476 & 2.154301 \\
\hline C & -3.945246 & 0.918403 & 3.088059 \\
\hline C & -5.076910 & -0.791837 & 1.823961 \\
\hline $\mathrm{H}$ & -4.194557 & -1.284571 & -0.062071 \\
\hline $\mathrm{C}$ & -2.852077 & -2.268456 & -3.949993 \\
\hline $\mathrm{H}$ & -1.814592 & -3.822560 & -2.920534 \\
\hline $\mathrm{C}$ & -1.600918 & -3.614787 & 0.227012 \\
\hline $\mathrm{C}$ & 0.248150 & -2.360913 & -0.622434 \\
\hline $\mathrm{H}$ & -3.735264 & -0.514110 & -4.805984 \\
\hline $\mathrm{C}$ & -3.105833 & 4.778510 & -1.507949 \\
\hline $\mathrm{H}$ & -5.099202 & 4.096890 & -1.569008 \\
\hline $\mathrm{H}$ & -0.999788 & 5.207003 & -1.345596 \\
\hline $\mathrm{C}$ & -4.988445 & -0.001369 & 2.969926 \\
\hline $\mathrm{H}$ & -3.878183 & 1.552222 & 3.962774 \\
\hline $\mathrm{H}$ & -5.895749 & -1.489665 & 1.709038 \\
\hline $\mathrm{H}$ & -3.063854 & -2.886395 & -4.813054 \\
\hline $\mathrm{C}$ & -0.729409 & -4.252118 & 1.128001 \\
\hline $\mathrm{N}$ & -2.996100 & -3.968602 & 0.241321 \\
\hline $\mathrm{C}$ & 1.100260 & -3.003996 & 0.286837 \\
\hline $\mathrm{H}$ & 0.681903 & -1.662968 & -1.320908 \\
\hline
\end{tabular}




\begin{tabular}{lrrr}
$\mathrm{H}$ & -3.371929 & 5.797621 & -1.757900 \\
$\mathrm{H}$ & -5.730786 & -0.088867 & 3.752518 \\
$\mathrm{C}$ & 0.627314 & -3.924926 & 1.136636 \\
$\mathrm{H}$ & -1.100029 & -5.003033 & 1.813955 \\
$\mathrm{C}$ & -3.729582 & -3.884780 & 1.504597 \\
$\mathrm{C}$ & -3.526779 & -4.865011 & -0.786532 \\
$\mathrm{H}$ & 2.151922 & -2.762531 & 0.296807 \\
$\mathrm{H}$ & 1.297221 & -4.416804 & 1.830322 \\
$\mathrm{H}$ & -4.798609 & -3.664031 & 1.305472 \\
$\mathrm{H}$ & -3.659035 & -4.851681 & 2.046161 \\
$\mathrm{H}$ & -3.308286 & -3.084630 & 2.150352 \\
$\mathrm{H}$ & -4.036549 & -5.734121 & -0.317986 \\
$\mathrm{H}$ & -4.257966 & -4.315987 & -1.415516 \\
$\mathrm{H}$ & -2.713648 & -5.270735 & -1.422617 \\
$\mathrm{H}$ & 5.591304 & -1.184429 & 1.963192 \\
$\mathrm{Si}$ & 5.091525 & -2.269905 & -0.803610 \\
$\mathrm{C}$ & 5.176990 & -3.888023 & 0.195290 \\
$\mathrm{H}$ & 5.727649 & -3.754835 & 1.134802 \\
$\mathrm{H}$ & 4.174397 & -4.249810 & 0.451919 \\
$\mathrm{H}$ & 5.682392 & -4.678921 & -0.374538 \\
$\mathrm{C}$ & 4.088493 & -2.570870 & -2.381317 \\
$\mathrm{H}$ & 3.057484 & -2.867630 & -2.157734 \\
$\mathrm{H}$ & 4.033512 & -1.670021 & -3.002888 \\
$\mathrm{H}$ & 4.544042 & -3.366950 & -2.984642 \\
$\mathrm{C}$ & 6.868591 & -1.792558 & -1.287688 \\
$\mathrm{H}$ & 7.356061 & -2.587212 & -1.867879 \\
$\mathrm{H}$ & 6.872911 & -0.880427 & -1.896303 \\
$\mathrm{H}$ & 7.486683 & -1.598687 & -0.402167 \\
$\mathrm{H}$ & 2.875537 & -0.289687 & -1.258086 \\
\hline \hline & & &
\end{tabular}

Table S50: Cartesian coordinates $(\AA)$ of t2sR (E= 1697.409061684719 a.u.).

\begin{tabular}{lrrr}
\hline \hline $\mathrm{C}$ & 1.117935 & -1.781230 & 3.151171 \\
$\mathrm{C}$ & 0.756561 & -1.349093 & 1.870823 \\
$\mathrm{C}$ & 0.883986 & -0.009554 & 1.485634 \\
$\mathrm{C}$ & 1.371357 & 0.932672 & 2.414789 \\
$\mathrm{C}$ & 1.719565 & 0.482847 & 3.699890 \\
$\mathrm{C}$ & 1.598387 & -0.855271 & 4.075237 \\
$\mathrm{Pd}$ & 0.305845 & 0.547373 & -0.396873 \\
$\mathrm{C}$ & 1.586122 & 2.381408 & 2.051442 \\
$\mathrm{C}$ & 2.970039 & 2.612025 & 1.378091 \\
$\mathrm{C}$ & 3.239805 & 1.604960 & 0.296290 \\
$\mathrm{C}$ & 2.462723 & 1.630413 & -0.890164 \\
$\mathrm{C}$ & 2.692035 & 0.661564 & -1.889961 \\
$\mathrm{C}$ & 3.673803 & -0.318973 & -1.716196 \\
$\mathrm{C}$ & 4.453683 & -0.361298 & -0.549198 \\
$\mathrm{C}$ & 4.211856 & 0.611935 & 0.439906 \\
$\mathrm{H}$ & 0.344442 & -2.071455 & 1.180137 \\
$\mathrm{H}$ & 4.796494 & 0.601384 & 1.357519 \\
$\mathrm{H}$ & 2.089187 & 1.212481 & 4.419026
\end{tabular}




\begin{tabular}{|c|c|c|c|}
\hline $\mathrm{H}$ & 1.867789 & -1.167109 & 5.082221 \\
\hline $\mathrm{H}$ & 1.004771 & -2.829983 & 3.419443 \\
\hline $\mathrm{H}$ & 1.859092 & 2.524171 & -1.081431 \\
\hline $\mathrm{H}$ & 1.527113 & 3.020183 & 2.938777 \\
\hline $\mathrm{H}$ & 0.820146 & 2.762826 & 1.372449 \\
\hline $\mathrm{H}$ & 3.763422 & 2.556754 & 2.135552 \\
\hline $\mathrm{H}$ & 2.931325 & 3.614879 & 0.933380 \\
\hline $\mathrm{Br}$ & -0.631948 & 1.225498 & -2.834312 \\
\hline $\mathrm{O}$ & 1.890935 & 4.692072 & -0.679624 \\
\hline $\mathrm{C}$ & 1.076806 & 5.371655 & 0.012491 \\
\hline $\mathrm{O}$ & 0.612988 & 5.179037 & 1.147302 \\
\hline $\mathrm{O}$ & 0.634419 & 6.557773 & -0.628471 \\
\hline $\mathrm{H}$ & 1.084929 & 6.503180 & -1.487080 \\
\hline $\mathrm{P}$ & -1.941323 & 0.267737 & 0.074066 \\
\hline $\mathrm{C}$ & -2.854043 & -0.687801 & -1.215357 \\
\hline $\mathrm{C}$ & -2.689843 & 1.932225 & 0.163892 \\
\hline $\mathrm{C}$ & -2.460872 & -0.389840 & 1.703854 \\
\hline $\mathrm{C}$ & -2.434287 & -1.984772 & -1.641419 \\
\hline $\mathrm{C}$ & -3.972456 & -0.102523 & -1.852169 \\
\hline $\mathrm{C}$ & -4.007257 & 2.106282 & 0.635304 \\
\hline $\mathrm{C}$ & -1.950487 & 3.018493 & -0.146811 \\
\hline $\mathrm{C}$ & -2.020327 & 0.219269 & 2.825578 \\
\hline C & -3.422640 & -1.412724 & 1.801458 \\
\hline $\mathrm{C}$ & -3.122826 & -2.601386 & -2.699788 \\
\hline $\mathrm{C}$ & -1.198122 & -2.661082 & -1.168226 \\
\hline $\mathrm{C}$ & -4.642138 & -0.736519 & -2.825545 \\
\hline $\mathrm{H}$ & -4.311171 & 0.884926 & -1.592906 \\
\hline $\mathrm{H}$ & -4.613637 & 1.254902 & 0.916523 \\
\hline $\mathrm{C}$ & -4.542769 & 3.395895 & 0.727449 \\
\hline $\mathrm{C}$ & -2.471019 & 4.259114 & -0.059176 \\
\hline $\mathrm{H}$ & -0.923823 & 2.904755 & -0.468211 \\
\hline $\mathrm{H}$ & -1.344018 & 1.059860 & 2.759421 \\
\hline $\mathrm{C}$ & -2.451187 & -0.192645 & 4.092295 \\
\hline C & -3.892688 & -1.819535 & 3.055208 \\
\hline $\mathrm{H}$ & -3.835240 & -1.878131 & 0.918487 \\
\hline $\mathrm{C}$ & -4.235912 & -1.991513 & -3.269737 \\
\hline $\mathrm{H}$ & -2.778034 & -3.542006 & -3.109424 \\
\hline $\mathrm{C}$ & -1.231592 & -3.806805 & -0.321581 \\
\hline C & -0.018376 & -2.285322 & -1.712234 \\
\hline $\mathrm{H}$ & -5.494157 & -0.257771 & -3.291958 \\
\hline $\mathrm{C}$ & -3.789580 & 4.452005 & 0.381941 \\
\hline $\mathrm{H}$ & -5.557496 & 3.539465 & 1.076035 \\
\hline $\mathrm{H}$ & -1.868519 & 5.117071 & -0.325986 \\
\hline $\mathrm{C}$ & -3.390369 & -1.218296 & 4.209026 \\
\hline $\mathrm{H}$ & -2.074585 & 0.300669 & 4.978937 \\
\hline $\mathrm{H}$ & -4.644646 & -2.593909 & 3.131108 \\
\hline $\mathrm{H}$ & -4.766127 & -2.478091 & -4.078206 \\
\hline C & -0.031146 & -4.495820 & -0.067435 \\
\hline $\mathrm{N}$ & -2.448611 & -4.276704 & 0.290136 \\
\hline $\mathrm{C}$ & 1.166583 & -2.984769 & -1.444091 \\
\hline $\mathrm{H}$ & 0.025066 & -1.462310 & -2.408618 \\
\hline
\end{tabular}




\begin{tabular}{lrrr}
$\mathrm{H}$ & -4.195527 & 5.453252 & 0.449709 \\
$\mathrm{H}$ & -3.740584 & -1.530117 & 5.184364 \\
$\mathrm{C}$ & 1.161105 & -4.060776 & -0.646665 \\
$\mathrm{H}$ & -0.021074 & -5.373922 & 0.564869 \\
$\mathrm{C}$ & -2.452946 & -4.548372 & 1.728918 \\
$\mathrm{C}$ & -3.457413 & -4.954426 & -0.525397 \\
$\mathrm{H}$ & 2.090139 & -2.668677 & -1.907104 \\
$\mathrm{H}$ & 2.079206 & -4.600929 & -0.456698 \\
$\mathrm{H}$ & -3.489847 & -4.506351 & 2.121573 \\
$\mathrm{H}$ & -2.044983 & -5.562518 & 1.923230 \\
$\mathrm{H}$ & -1.833334 & -3.799404 & 2.267488 \\
$\mathrm{H}$ & -3.733650 & -5.930060 & -0.070687 \\
$\mathrm{H}$ & -4.365431 & -4.319967 & -0.594462 \\
$\mathrm{H}$ & -3.070443 & -5.171260 & -1.541391 \\
$\mathrm{H}$ & 2.123192 & 0.715876 & -2.811962 \\
$\mathrm{Si}$ & 5.762614 & -1.702441 & -0.321554 \\
$\mathrm{H}$ & 3.838628 & -1.041119 & -2.514396 \\
$\mathrm{C}$ & 6.965142 & -1.678325 & -1.791954 \\
$\mathrm{H}$ & 7.714698 & -2.475999 & -1.708963 \\
$\mathrm{H}$ & 6.436829 & -1.817291 & -2.742933 \\
$\mathrm{H}$ & 7.497624 & -0.721495 & -1.849932 \\
$\mathrm{C}$ & 6.737826 & -1.413446 & 1.278754 \\
$\mathrm{H}$ & 7.244711 & -0.441169 & 1.274128 \\
$\mathrm{H}$ & 6.085780 & -1.443811 & 2.159617 \\
$\mathrm{H}$ & 7.505187 & -2.187369 & 1.407893 \\
$\mathrm{C}$ & 4.933393 & -3.407109 & -0.239487 \\
$\mathrm{H}$ & 4.197465 & -3.442081 & 0.572354 \\
$\mathrm{H}$ & 4.406404 & -3.637143 & -1.173067 \\
$\mathrm{H}$ & 5.668322 & -4.203999 & -0.066733 \\
\hline & & & \\
\hline
\end{tabular}

Table S51: Cartesian coordinates $(\AA)$ of t2sTS $(E=-$ 1697.392028244141 a.u.).

\begin{tabular}{lrrr}
\hline \hline $\mathrm{C}$ & 0.768592 & -2.046100 & 3.133624 \\
$\mathrm{C}$ & 0.523118 & -1.461015 & 1.886786 \\
$\mathrm{C}$ & 0.727077 & -0.094184 & 1.656239 \\
$\mathrm{C}$ & 1.213856 & 0.697308 & 2.715598 \\
$\mathrm{C}$ & 1.446145 & 0.104838 & 3.966295 \\
$\mathrm{C}$ & 1.224646 & -1.255571 & 4.186737 \\
$\mathrm{Pd}$ & 0.233832 & 0.720611 & -0.154514 \\
$\mathrm{C}$ & 1.575311 & 2.143111 & 2.486867 \\
$\mathrm{C}$ & 2.989466 & 2.275359 & 1.869694 \\
$\mathrm{C}$ & 3.273038 & 1.343443 & 0.710432 \\
$\mathrm{C}$ & 2.383828 & 1.278483 & -0.398077 \\
$\mathrm{C}$ & 2.791378 & 0.513541 & -1.515508 \\
$\mathrm{C}$ & 3.977288 & -0.219969 & -1.514597 \\
$\mathrm{C}$ & 4.822030 & -0.216958 & -0.390508 \\
$\mathrm{C}$ & 4.455860 & 0.597655 & 0.697917 \\
$\mathrm{H}$ & 0.133601 & -2.084511 & 1.093787 \\
$\mathrm{H}$ & 5.106895 & 0.649768 & 1.569982 \\
$\mathrm{H}$ & 1.812318 & 0.730057 & 4.780220
\end{tabular}




$\begin{array}{lrrr}\mathrm{H} & 1.405957 & -1.688976 & 5.168300 \\ \mathrm{H} & 0.587960 & -3.110428 & 3.275351 \\ \mathrm{H} & 1.905430 & 2.494753 & -0.685574 \\ \mathrm{H} & 1.556625 & 2.704921 & 3.429460 \\ \mathrm{H} & 0.859117 & 2.628288 & 1.820299 \\ \mathrm{H} & 3.744902 & 2.111171 & 2.650232 \\ \mathrm{H} & 3.079669 & 3.312922 & 1.519931 \\ \mathrm{Br} & -0.605116 & 1.670513 & -2.556003 \\ \mathrm{O} & 1.821099 & 3.693673 & -1.141044 \\ \mathrm{C} & 1.172926 & 4.576823 & -0.442150 \\ \mathrm{O} & 1.321640 & 4.892948 & 0.734193 \\ \mathrm{O} & 0.205036 & 5.224794 & -1.182803 \\ \mathrm{H} & 0.132720 & 4.689747 & -1.994050 \\ \mathrm{P} & -2.077577 & 0.285283 & 0.102308 \\ \mathrm{C} & -2.880672 & -0.510568 & -1.357985 \\ \mathrm{C} & -2.887671 & 1.900656 & 0.368973 \\ \mathrm{C} & -2.689943 & -0.584959 & 1.595450 \\ \mathrm{C} & -2.41140 & -1.745133 & -1.899707 \\ \mathrm{C} & -3.959512 & 0.137469 & -2.001676 \\ \mathrm{C} & -4.246922 & 1.983743 & 0.733354 \\ \mathrm{C} & -2.146470 & 3.026815 & 0.320164 \\ \mathrm{C} & -2.304387 & -0.145020 & 2.812457 \\ \mathrm{C} & -3.666798 & -1.593597 & 1.498277 \\ \mathrm{C} & -3.022689 & -2.247735 & -3.060548 \\ \mathrm{C} & -0.015898 & -4.341528 & -0.452704 \\ \mathrm{C} & -1.182893 & -2.441902 & -1.436706 \\ \mathrm{H} & 1.201985 & -2.672001 & -1.610974 \\ \mathrm{C} & -4.555498 & -0.389195 & -3.081303 \\ \mathrm{H} & -4.323939 & 1.090439 & -1.660850 \\ \mathrm{H} & -4.856212 & 1.092623 & 0.812628 \\ \mathrm{C} & -4.816616 & 3.236655 & 0.988396 \\ \mathrm{C} & -2.696414 & 4.231610 & 0.570555 \\ \mathrm{H} & -1.092068 & 2.968984 & 0.089826 \\ \mathrm{H} & -1.610666 & 0.679981 & 2.899471 \\ \mathrm{C} & -2.810395 & -0.719198 & 3.984860 \\ \mathrm{C} & -4.211013 & -2.159795 & 2.656283 \\ \mathrm{H} & -4.033068 & -1.926632 & 0.538233 \\ \mathrm{C} & -4.106277 & -1.586807 & -3.630899 \\ \mathrm{H} & -2.637413 & -3.133629 & -3.548654 \\ \mathrm{C} & -1.224281 & -3.665949 & -0.708845 \\ \mathrm{C} & 0.010439 & -1.982228 & -1.875833 \\ \mathrm{H} & -5.380180 & 0.134008 & -3.549060 \\ \mathrm{H} & -4.055760 & 4.340069 & 0.904821 \\ \mathrm{H} & -2.084576 & 3.313620 & 1.256533 \\ \mathrm{H} & -4.123338 & 0.524249 \\ \mathrm{H} & & -1.732478 & 3.907659 \\ \mathrm{H} & -0.361522 & 4.950347 \\ \mathrm{H} & -2671 & -2.924956 & 2.582816 \\ \mathrm{H} & -1.483956\end{array}$




\begin{tabular}{rrrr}
$\mathrm{H}$ & -4.486902 & 5.312713 & 1.104707 \\
$\mathrm{H}$ & -4.174143 & -2.169535 & 4.810158 \\
$\mathrm{C}$ & 1.191041 & -3.817397 & -0.917685 \\
$\mathrm{H}$ & -0.009405 & -5.275505 & 0.093601 \\
$\mathrm{C}$ & -2.526281 & -4.650282 & 1.185129 \\
$\mathrm{C}$ & -3.409121 & -4.826036 & -1.149378 \\
$\mathrm{H}$ & 2.138062 & -2.290166 & -1.991979 \\
$\mathrm{H}$ & 2.117884 & -4.343011 & -0.725525 \\
$\mathrm{H}$ & -3.583233 & -4.688187 & 1.520780 \\
$\mathrm{H}$ & -2.087754 & -5.664288 & 1.294626 \\
$\mathrm{H}$ & -1.968372 & -3.941216 & 1.833901 \\
$\mathrm{H}$ & -3.681356 & -5.852092 & -0.820771 \\
$\mathrm{H}$ & -4.328503 & -4.205750 & -1.191646 \\
$\mathrm{H}$ & -2.969979 & -4.918613 & -2.163066 \\
$\mathrm{H}$ & 2.158447 & 0.513736 & -2.399405 \\
$\mathrm{Si}$ & 6.345359 & -1.321779 & -0.358641 \\
$\mathrm{H}$ & 4.238425 & -0.809158 & -2.394145 \\
$\mathrm{C}$ & 7.504071 & -0.900849 & -1.805268 \\
$\mathrm{H}$ & 8.373962 & -1.570151 & -1.830594 \\
$\mathrm{H}$ & 6.987536 & -0.989307 & -2.768587 \\
$\mathrm{H}$ & 7.873566 & 0.128574 & -1.725794 \\
$\mathrm{C}$ & 7.301442 & -1.114866 & 1.267342 \\
$\mathrm{H}$ & 7.654038 & -0.085063 & 1.400758 \\
$\mathrm{H}$ & 6.678912 & -1.366656 & 2.134022 \\
$\mathrm{H}$ & 8.180341 & -1.771954 & 1.286957 \\
$\mathrm{C}$ & 5.797972 & -3.133968 & -0.525918 \\
$\mathrm{H}$ & 5.149489 & -3.421259 & 0.310400 \\
$\mathrm{H}$ & 5.225357 & -3.289440 & -1.448329 \\
$\mathrm{H}$ & 6.654698 & -3.820299 & -0.542374 \\
\hline \hline & & &
\end{tabular}

Table S52: Cartesian coordinates $(\AA)$ of $\mathbf{t 2 t R}(\mathrm{E}=$ 1697.404042793155 a.u.).

\begin{tabular}{lrrr}
\hline \hline $\mathrm{C}$ & 0.490294 & -2.619331 & 2.583534 \\
$\mathrm{C}$ & 0.202054 & -1.920298 & 1.407157 \\
$\mathrm{C}$ & 0.844100 & -0.717094 & 1.088105 \\
$\mathrm{C}$ & 1.817738 & -0.204557 & 1.969601 \\
$\mathrm{C}$ & 2.075054 & -0.905615 & 3.160931 \\
$\mathrm{C}$ & 1.423916 & -2.097978 & 3.476956 \\
$\mathrm{Pd}$ & 0.261684 & 0.331669 & -0.567153 \\
$\mathrm{C}$ & 2.651082 & 1.009847 & 1.637869 \\
$\mathrm{C}$ & 3.880886 & 0.633451 & 0.766029 \\
$\mathrm{C}$ & 3.515473 & -0.091276 & -0.503490 \\
$\mathrm{C}$ & 2.576037 & 0.545995 & -1.365680 \\
$\mathrm{C}$ & 2.210128 & -0.053674 & -2.587211 \\
$\mathrm{C}$ & 2.739882 & -1.294663 & -2.944058 \\
$\mathrm{C}$ & 3.664782 & -1.904683 & -2.102901 \\
$\mathrm{C}$ & 4.085612 & -1.329494 & -0.882284 \\
$\mathrm{H}$ & -0.566560 & -2.310063 & 0.754848 \\
$\mathrm{H}$ & 2.810769 & -0.495880 & 3.851674 \\
$\mathrm{H}$ & 1.643492 & -2.610170 & 4.411210
\end{tabular}




\begin{tabular}{|c|c|c|c|}
\hline $\mathrm{H}$ & -0.032449 & -3.548761 & 2.801300 \\
\hline $\mathrm{H}$ & 2.363854 & 1.609035 & -1.172433 \\
\hline $\mathrm{H}$ & 3.028629 & 1.476160 & 2.555778 \\
\hline $\mathrm{H}$ & 2.089048 & 1.781917 & 1.105018 \\
\hline $\mathrm{H}$ & 4.565325 & 0.026463 & 1.368059 \\
\hline $\mathrm{H}$ & 4.378754 & 1.593429 & 0.531514 \\
\hline $\mathrm{Br}$ & -0.745536 & 1.775395 & -2.640122 \\
\hline $\mathrm{O}$ & 2.630944 & 3.499437 & -0.392473 \\
\hline $\mathrm{C}$ & 3.670381 & 4.077702 & 0.033972 \\
\hline $\mathrm{O}$ & 4.727596 & 3.615143 & 0.498671 \\
\hline $\mathrm{O}$ & 3.609828 & 5.489621 & -0.026419 \\
\hline $\mathrm{H}$ & 2.734210 & 5.638768 & -0.419962 \\
\hline $\mathrm{P}$ & -1.825297 & 0.808035 & 0.323704 \\
\hline $\mathrm{C}$ & -3.221210 & 0.486774 & -0.839861 \\
\hline $\mathrm{C}$ & -1.830354 & 2.585938 & 0.741713 \\
\hline $\mathrm{C}$ & -2.307293 & 0.119129 & 1.952423 \\
\hline $\mathrm{C}$ & -3.398305 & -0.777428 & -1.480242 \\
\hline $\mathrm{C}$ & -4.114011 & 1.533727 & -1.162803 \\
\hline C & -2.891594 & 3.158631 & 1.471488 \\
\hline $\mathrm{C}$ & -0.751420 & 3.339738 & 0.443909 \\
\hline $\mathrm{C}$ & -1.506258 & 0.336772 & 3.017840 \\
\hline C & -3.556311 & -0.501888 & 2.137732 \\
\hline $\mathrm{C}$ & -4.426062 & -0.913620 & -2.428356 \\
\hline $\mathrm{C}$ & -2.454523 & -1.918439 & -1.346309 \\
\hline $\mathrm{C}$ & -5.117457 & 1.360650 & -2.035247 \\
\hline $\mathrm{H}$ & -4.003251 & 2.514772 & -0.736182 \\
\hline $\mathrm{H}$ & -3.752496 & 2.567142 & 1.755793 \\
\hline $\mathrm{C}$ & -2.832042 & 4.510901 & 1.828492 \\
\hline C & -0.694675 & 4.640504 & 0.791789 \\
\hline $\mathrm{H}$ & 0.089380 & 2.899220 & -0.072377 \\
\hline $\mathrm{H}$ & -0.576784 & 0.876920 & 2.899708 \\
\hline C & -1.866090 & -0.102056 & 4.297653 \\
\hline $\mathrm{C}$ & -3.950295 & -0.923214 & 3.412673 \\
\hline $\mathrm{H}$ & -4.239306 & -0.640575 & 1.312559 \\
\hline $\mathrm{C}$ & -5.296039 & 0.141958 & -2.684084 \\
\hline $\mathrm{H}$ & -4.534102 & -1.826678 & -2.998803 \\
\hline C & -2.799300 & -3.115372 & -0.655243 \\
\hline $\mathrm{C}$ & -1.297204 & -1.878028 & -2.044788 \\
\hline $\mathrm{H}$ & -5.783014 & 2.184864 & -2.259643 \\
\hline $\mathrm{C}$ & -1.754957 & 5.238734 & 1.491249 \\
\hline $\mathrm{H}$ & -3.647071 & 4.964255 & 2.377982 \\
\hline $\mathrm{H}$ & 0.178963 & 5.227786 & 0.538860 \\
\hline $\mathrm{C}$ & -3.092710 & -0.737205 & 4.496851 \\
\hline $\mathrm{H}$ & -1.202549 & 0.069804 & 5.135236 \\
\hline $\mathrm{H}$ & -4.916246 & -1.388135 & 3.559292 \\
\hline $\mathrm{H}$ & -6.090815 & 0.025791 & -3.409639 \\
\hline $\mathrm{C}$ & -1.912651 & -4.206718 & -0.706008 \\
\hline $\mathrm{N}$ & -4.013645 & -3.238573 & 0.109285 \\
\hline $\mathrm{C}$ & -0.425139 & -2.975395 & -2.079233 \\
\hline $\mathrm{H}$ & -1.040689 & -1.007397 & -2.627999 \\
\hline $\mathrm{H}$ & -1.698622 & 6.284086 & 1.766865 \\
\hline
\end{tabular}




\begin{tabular}{lrrr}
$\mathrm{H}$ & -3.383479 & -1.065760 & 5.486119 \\
$\mathrm{C}$ & -0.723362 & -4.108040 & -1.429554 \\
$\mathrm{H}$ & -2.145688 & -5.131078 & -0.193656 \\
$\mathrm{C}$ & -3.927928 & -3.704688 & 1.494295 \\
$\mathrm{C}$ & -5.291828 & -3.413171 & -0.581650 \\
$\mathrm{H}$ & 0.488197 & -2.915785 & -2.653590 \\
$\mathrm{H}$ & -0.044701 & -4.950488 & -1.469510 \\
$\mathrm{H}$ & -4.806078 & -3.346640 & 2.070368 \\
$\mathrm{H}$ & -3.917393 & -4.814686 & 1.518953 \\
$\mathrm{H}$ & -3.001411 & -3.325351 & 1.976483 \\
$\mathrm{H}$ & -5.822122 & -4.310383 & -0.196145 \\
$\mathrm{H}$ & -5.928414 & -2.519576 & -0.414350 \\
$\mathrm{H}$ & -5.140976 & -3.569794 & -1.668931 \\
$\mathrm{H}$ & 4.085070 & -2.858699 & -2.411688 \\
$\mathrm{H}$ & 1.544005 & 0.479812 & -3.255942 \\
$\mathrm{Si}$ & 5.470150 & -2.219830 & 0.068260 \\
$\mathrm{C}$ & 7.011095 & -1.120249 & 0.216313 \\
$\mathrm{H}$ & 6.806281 & -0.182575 & 0.742637 \\
$\mathrm{H}$ & 7.813405 & -1.643155 & 0.753888 \\
$\mathrm{H}$ & 7.392301 & -0.856986 & -0.777956 \\
$\mathrm{C}$ & 4.944296 & -2.841803 & 1.782300 \\
$\mathrm{H}$ & 4.710826 & -2.026807 & 2.473223 \\
$\mathrm{H}$ & 4.047133 & -3.467983 & 1.713071 \\
$\mathrm{H}$ & 5.746805 & -3.445627 & 2.227277 \\
$\mathrm{C}$ & 5.995148 & -3.764641 & -0.917070 \\
$\mathrm{H}$ & 6.359972 & -3.516241 & -1.920951 \\
$\mathrm{H}$ & 6.812255 & -4.272692 & -0.388368 \\
$\mathrm{H}$ & 5.178323 & -4.488544 & -1.026963 \\
$\mathrm{H}$ & 2.455778 & -1.764768 & -3.882580 \\
\hline \hline & & &
\end{tabular}

Table S53: Cartesian coordinates $(\AA)$ of t2tTS $(E=-$ 1697.386143542533 a.u.).

\begin{tabular}{lrrr}
\hline \hline $\mathrm{C}$ & 0.704092 & -2.925034 & 2.280163 \\
$\mathrm{C}$ & 0.385707 & -2.077713 & 1.212971 \\
$\mathrm{C}$ & 0.929084 & -0.791063 & 1.103951 \\
$\mathrm{C}$ & 1.838710 & -0.361918 & 2.089630 \\
$\mathrm{C}$ & 2.138215 & -1.210517 & 3.166384 \\
$\mathrm{C}$ & 1.578040 & -2.485182 & 3.272413 \\
$\mathrm{Pd}$ & 0.320851 & 0.476682 & -0.375212 \\
$\mathrm{C}$ & 2.542444 & 0.963342 & 1.946470 \\
$\mathrm{C}$ & 3.773637 & 0.858086 & 1.013085 \\
$\mathrm{C}$ & 3.558452 & 0.137992 & -0.306582 \\
$\mathrm{C}$ & 2.436637 & 0.516651 & -1.101312 \\
$\mathrm{C}$ & 2.327882 & -0.016561 & -2.405491 \\
$\mathrm{C}$ & 3.249757 & -0.939992 & -2.893079 \\
$\mathrm{C}$ & 4.316401 & -1.324025 & -2.083972 \\
$\mathrm{C}$ & 4.514471 & -0.788701 & -0.792687 \\
$\mathrm{H}$ & -0.330215 & -2.421766 & 0.478706 \\
$\mathrm{H}$ & 2.825117 & -0.857913 & 3.935368 \\
$\mathrm{H}$ & 1.821317 & -3.122314 & 4.120172
\end{tabular}




\begin{tabular}{|c|c|c|c|}
\hline $\mathrm{H}$ & 0.253186 & -3.914436 & 2.336484 \\
\hline $\mathrm{H}$ & 2.278502 & 1.833814 & -1.038618 \\
\hline $\mathrm{H}$ & 2.889539 & 1.328918 & 2.921346 \\
\hline $\mathrm{H}$ & 1.867877 & 1.726396 & 1.554239 \\
\hline $\mathrm{H}$ & 4.586946 & 0.381582 & 1.568936 \\
\hline $\mathrm{H}$ & 4.079327 & 1.891347 & 0.793910 \\
\hline $\mathrm{Br}$ & -0.705767 & 2.106373 & -2.299353 \\
\hline $\mathrm{O}$ & 2.468447 & 3.098136 & -1.271630 \\
\hline $\mathrm{C}$ & 2.277931 & 3.963333 & -0.322423 \\
\hline $\mathrm{O}$ & 2.768751 & 3.998572 & 0.802339 \\
\hline $\mathrm{O}$ & 1.404071 & 4.966459 & -0.691959 \\
\hline $\mathrm{H}$ & 1.006309 & 4.637343 & -1.518495 \\
\hline $\mathrm{P}$ & -1.905586 & 0.602736 & 0.427505 \\
\hline $\mathrm{C}$ & -3.205219 & 0.358628 & -0.861277 \\
\hline $\mathrm{C}$ & -2.123308 & 2.277336 & 1.124099 \\
\hline $\mathrm{C}$ & -2.408298 & -0.377395 & 1.893268 \\
\hline $\mathrm{C}$ & -3.251269 & -0.813903 & -1.673748 \\
\hline $\mathrm{C}$ & -4.154488 & 1.378434 & -1.099861 \\
\hline C & -3.285229 & 2.621910 & 1.844132 \\
\hline $\mathrm{C}$ & -1.110965 & 3.164939 & 1.039152 \\
\hline $\mathrm{C}$ & -1.657312 & -0.308861 & 3.013365 \\
\hline C & -3.633696 & -1.069229 & 1.919091 \\
\hline C & -4.217587 & -0.891826 & -2.690632 \\
\hline $\mathrm{C}$ & -2.216286 & -1.879824 & -1.649944 \\
\hline $\mathrm{C}$ & -5.096829 & 1.256744 & -2.046135 \\
\hline $\mathrm{H}$ & -4.137128 & 2.300201 & -0.545549 \\
\hline $\mathrm{H}$ & -4.098788 & 1.916770 & 1.956093 \\
\hline C & -3.389877 & 3.895756 & 2.414868 \\
\hline $\mathrm{C}$ & -1.212388 & 4.389290 & 1.593570 \\
\hline $\mathrm{H}$ & -0.196713 & 2.893135 & 0.531011 \\
\hline $\mathrm{H}$ & -0.750841 & 0.280763 & 3.026765 \\
\hline C & -2.042813 & -0.968831 & 4.186395 \\
\hline $\mathrm{C}$ & -4.055423 & -1.709511 & 3.089589 \\
\hline $\mathrm{H}$ & -4.279672 & -1.094868 & 1.053734 \\
\hline $\mathrm{C}$ & -5.151848 & 0.126056 & -2.856610 \\
\hline $\mathrm{H}$ & -4.225713 & -1.723862 & -3.382608 \\
\hline C & -2.468553 & -3.180883 & -1.127733 \\
\hline $\mathrm{C}$ & -1.053116 & -1.649802 & -2.299511 \\
\hline $\mathrm{H}$ & -5.809014 & 2.057490 & -2.201798 \\
\hline $\mathrm{C}$ & -2.373361 & 4.764135 & 2.288473 \\
\hline $\mathrm{H}$ & -4.282762 & 4.175764 & 2.959009 \\
\hline $\mathrm{H}$ & -0.389232 & 5.087474 & 1.512497 \\
\hline $\mathrm{C}$ & -3.246650 & -1.674021 & 4.225465 \\
\hline $\mathrm{H}$ & -1.418684 & -0.913076 & 5.068850 \\
\hline $\mathrm{H}$ & -5.004319 & -2.229002 & 3.115244 \\
\hline $\mathrm{H}$ & -5.897999 & 0.054886 & -3.637476 \\
\hline $\mathrm{C}$ & -1.487184 & -4.176704 & -1.290049 \\
\hline $\mathrm{N}$ & -3.684588 & -3.505477 & -0.426380 \\
\hline $\mathrm{C}$ & -0.088034 & -2.655671 & -2.449054 \\
\hline $\mathrm{H}$ & -0.864987 & -0.690207 & -2.757327 \\
\hline $\mathrm{H}$ & -2.443817 & 5.749855 & 2.730361 \\
\hline
\end{tabular}




\begin{tabular}{lrrr}
$\mathrm{H}$ & -3.558260 & -2.173056 & 5.133770 \\
$\mathrm{C}$ & -0.297675 & -3.884150 & -1.958768 \\
$\mathrm{H}$ & -1.646852 & -5.177193 & -0.909909 \\
$\mathrm{C}$ & -3.591077 & -4.177534 & 0.871094 \\
$\mathrm{C}$ & -4.936608 & -3.648915 & -1.170515 \\
$\mathrm{H}$ & 0.825925 & -2.445550 & -2.986300 \\
$\mathrm{H}$ & 0.452565 & -4.653972 & -2.086920 \\
$\mathrm{H}$ & -4.517534 & -4.005677 & 1.457118 \\
$\mathrm{H}$ & -3.467434 & -5.270783 & 0.722320 \\
$\mathrm{H}$ & -2.723619 & -3.790049 & 1.447570 \\
$\mathrm{H}$ & -5.420253 & -4.620924 & -0.933481 \\
$\mathrm{H}$ & -5.628269 & -2.826093 & -0.894175 \\
$\mathrm{H}$ & -4.755221 & -3.637837 & -2.264171 \\
$\mathrm{H}$ & 5.027937 & -2.046347 & -2.477635 \\
$\mathrm{H}$ & 1.517406 & 0.331276 & -3.040410 \\
$\mathrm{Si}$ & 6.062729 & -1.351480 & 0.148648 \\
$\mathrm{C}$ & 7.253393 & 0.084398 & 0.521598 \\
$\mathrm{H}$ & 6.806416 & 0.854819 & 1.157684 \\
$\mathrm{H}$ & 8.156985 & -0.286387 & 1.024110 \\
$\mathrm{H}$ & 7.566625 & 0.572133 & -0.409798 \\
$\mathrm{C}$ & 5.643532 & -2.288597 & 1.748017 \\
$\mathrm{H}$ & 5.012106 & -1.710841 & 2.429435 \\
$\mathrm{H}$ & 5.096203 & -3.209558 & 1.512438 \\
$\mathrm{H}$ & 6.559304 & -2.570582 & 2.285011 \\
$\mathrm{C}$ & 7.049753 & -2.575244 & -0.928684 \\
$\mathrm{H}$ & 7.384102 & -2.125539 & -1.871397 \\
$\mathrm{H}$ & 7.946103 & -2.900657 & -0.384429 \\
$\mathrm{H}$ & 6.471689 & -3.474639 & -1.172818 \\
$\mathrm{H}$ & 3.147801 & -1.351102 & -3.895782 \\
\hline & & &
\end{tabular}

